TRANSACTIONS OF THE

AMERICAN MATHEMATICAL SOCIETY

Volume 360, Number 11, November 2008, Pages 5883-5924

S 0002-9947(08)04447-4

Article electronically published on June 26, 2008

\title{
SUPER DUALITY AND KAZHDAN-LUSZTIG POLYNOMIALS
}

\author{
SHUN-JEN CHENG, WEIQIANG WANG, AND R. B. ZHANG
}

\begin{abstract}
We establish a direct connection between the representation theories of Lie algebras and Lie superalgebras (of type $A$ ) via Fock space reformulations of their Kazhdan-Lusztig theories. As a consequence, the characters of finite-dimensional irreducible modules of the general linear Lie superalgebra are computed by the usual parabolic Kazhdan-Lusztig polynomials of type $A$. In addition, we establish closed formulas for canonical and dual canonical bases for the tensor product of any two fundamental representations of type A.
\end{abstract}

\section{INTRODUCTION}

1.1. Background. Since the classification of finite-dimensional complex simple Lie superalgebras by Kac [K1, there has been an enormous amount of literature devoted to the representation theory of Lie superalgebras, in particular that of the general linear superalgebras $\mathfrak{g l}(m \mid n)$ over $\mathbb{C}$ (cf. [BL, $\overline{\mathrm{BR}}, \mathrm{Br} 1, \overline{\mathrm{Br} 2}, \overline{\mathrm{CW}}$,, $\mathrm{CZ}$, JHKT, JZ, K2, Ku, PS, Se1, Se2, Sv, Zou and the references therein). While having many similarities with the representation theory of Lie algebras, it was soon realized however that the study of representations of Lie superalgebras is in general very different and difficult: often a given method borrowed from semisimple Lie algebras (e.g. Borel-Weil construction, Weyl characters, etc.) can only cover limited classes of representations. In the case when a weight $\lambda$ is typical, the irreducible highest weight module $L_{n}(\lambda)$ of $\mathfrak{g l}(m \mid n)$ coincides with the so-called Kac module, whose character was obtained in $\mathrm{K} 2$.

For the study of the representation theory of reductive Lie algebras, the KazhdanLusztig (KL) theory $\mathrm{KL}$ has proved to be an extremely powerful tool. The Kazhdan-Lusztig conjecture which expresses the characters of irreducible modules of a simple Lie algebra in terms of KL polynomials has been established independently in $[\mathrm{BB}, \overline{\mathrm{BK}}$. A decade ago, the idea of KL theory was applied by Serganova Se1, Se2 (with a mixture of algebraic and geometric techniques) to offer a complete solution to the longstanding problem of finding the characters of finite-dimensional irreducible modules of $\mathfrak{g l}(m \mid n)$.

Recently, partly inspired by the ideas of Lascoux, Leclerc and Thibon [LLT, Brundan [Br1] offered a very different, purely algebraic, elegant Fock space approach to the irreducible character problem for $\mathfrak{g l}(m \mid n)$. Brundan's Fock space 1

$$
\mathcal{E}^{m \mid n}:=\Lambda^{m} \mathbb{V} \otimes \Lambda^{n} \mathbb{V}^{*},
$$

Received by the editors October 17, 2006.

2000 Mathematics Subject Classification. Primary 17B10; Secondary 17B37, 20 C08.

${ }^{1}$ In [Br1], the roles of $\mathbb{V}$ and $\mathbb{V}^{*}$ are switched, but this is not essential. 
where $\mathbb{V}$ (respectively $\mathbb{V}^{*}$ ) is the natural (respectively dual) module of the quantum group $\mathcal{U}=U_{q}(\mathfrak{s l}(\infty))$, affords several bases such as the monomial basis and the Kashiwara-Lusztig (dual) canonical bases (Lu1, Kas, Lu2). Brundan computed the transition matrices of KL polynomials between the (dual) canonical basis and the monomial basis for $\mathcal{E}^{m \mid n}$, and further established in terms of the KL polynomials the character formulas of the irreducible modules and of the tilting modules in the category $\mathcal{O}_{m \mid n}^{+}$of finite-dimensional modules of $\mathfrak{g l}(m \mid n)$. (We will denote by $\mathrm{O}_{m \mid \infty}^{++}$an analogous category; see Section 3) His definition of KL polynomials was also shown to coincide with the one given originally in [Se1, Se2] and it has a cohomological interpretation in the sense of Vogan $\mathrm{Vo}$ (also cf. [CPS, [ Zou]).

The category $\mathcal{O}_{m \mid n}^{+}$is reminiscent of the parabolic category $\mathcal{O}_{m+n}^{+}$of $\mathfrak{g l}(m+n)$ modules which has been well studied in the literature (cf. e.g. [CC, Deo, ES]; see Section 5 for a precise definition, where an analogous category $\mathcal{O}_{m+\infty}^{++}$of $\mathfrak{g l}(m+\infty)$ modules is also defined). Kac modules of $\mathfrak{g l}(m \mid n)$ can be viewed as super analogues of generalized Verma modules. The differences between the categories $\mathcal{O}_{m \mid n}^{+}$and $\mathcal{O}_{m+n}^{+}$are however rather significant: a block in $\mathcal{O}_{m \mid n}^{+}$usually contains infinitely many simple objects, and it is controlled by the group $S_{m \mid n}=S_{m} \times S_{n}$ and odd reflections (as demonstrated in [Se1]). On the other hand, a block in $\mathcal{O}_{m+n}^{+}$contains finitely many objects and it is controlled by the Weyl group $S_{m+n}$. In the category $\mathcal{O}_{m+n}^{+}$, the tilting modules are usually different from the projective modules [CoI]. In contrast, the tilting modules in $\mathcal{O}_{m \mid n}^{+}$coincide with the projective covers of the simple modules $\mathrm{Br} 1$.

1.2. The main results. This paper provides for the first time a direct connection between the representation theories of Lie algebras and Lie superalgebras of type $A$ via a Fock space reformulation of the Kazhdan-Lusztig theory. Motivated by Br1 (also see [LLT]), we reformulate the KL theory for the category $\mathcal{O}_{m+n}^{+}$in terms of the canonical and dual canonical bases on the Fock space

$$
\mathcal{E}^{m+n}:=\Lambda^{m} \mathbb{V} \otimes \Lambda^{n} \mathbb{V}
$$

via the Schur-Jimbo duality Jim. Brundan, via personal communication, has informed us that he was aware of this reformulation. Our key starting point here is that one has to take the limit $n \rightarrow \infty$ in order to make a precise connection with the category $\mathcal{O}_{m \mid n}^{+}$. Let us summarize our main results.

- There exists a $\mathcal{U}$-module isomorphism of Fock spaces of semi-infinite $q$ wedges $\Lambda^{\infty} \mathbb{V} \cong \Lambda^{\infty} \mathbb{V}^{*}$. This induces a $\mathcal{U}$-module isomorphism

$$
\natural: \widehat{\mathcal{E}}^{m+\infty} \stackrel{\cong}{\longrightarrow} \widehat{\mathcal{E}}^{m \mid \infty},
$$

which commutes with the bar involutions on $\widehat{\mathcal{E}}^{m+\infty}$ and $\widehat{\mathcal{E}}^{m \mid \propto \alpha 2}$. It follows that the map $\llbracket$ sends the monomial, canonical, and dual canonical bases for $\widehat{\mathcal{E}}^{m+\infty}$ to the corresponding bases for $\widehat{\mathcal{E}}^{m \mid \infty}$ (Theorem 6.7).

- We obtain closed formulas for the transition matrices among the canonical, dual canonical, and the monomial bases in $\varepsilon^{m+n}$. Our formulas generalize those of Leclerc and Miyachi [LM] and our method is inspired by Br1, [LM. We introduce the truncation maps and use them to show that the

\footnotetext{
${ }^{2}$ The hat here stands for a certain topological completion. Here we will be slightly imprecise by ignoring various topological completions for the sake of simplicity. These will be made rigorous later on.
} 
combinatorics of the (dual) canonical bases for $\widehat{\mathcal{E}}^{m+\infty}$ (respectively $\widehat{\mathcal{E}}^{m \mid \infty}$ ) is equivalent to those of $\mathcal{E}^{m+n}$ (respectively $\mathcal{E}^{m \mid n}$ ) for all $n$. As a consequence, the Brundan-Serganova KL polynomials for $\mathfrak{g l}(m \mid n)$ are exactly the usual parabolic KL polynomials of type $A$. (See Remark 6.11)

- We establish an isomorphism of $\mathfrak{U}_{q=1}$-modules between $\left.\mathcal{E}^{m+n}\right|_{q=1}$ and the rational Grothendieck group of $\mathcal{O}_{m+n}^{+}$, where the Chevalley generators act via certain translation functors and the canonical, dual canonical, and monomial bases are identified with the tilting, irreducible, and generalized Verma modules, respectively.

- The tilting modules for the categories $\mathcal{O}_{m \mid n}^{+}$are shown to be compatible under the truncation functor (see Definition 3.4), and then are "glued" together into a module in the category $\mathcal{O}_{m \mid \infty}^{++}$which is shown to be a tilting module. There exists a natural isomorphism of the Grothendieck groups of $\mathcal{O}_{m+\infty}^{++}$and $\mathcal{O}_{m \mid \infty}^{++}$that matches the tilting, generalized Verma, and irreducible modules with the tilting, Kac, and irreducible modules, respectively. This isomorphism (called Super Duality) is shown to be compatible with tensor products. The categories $\mathcal{O}_{m+\infty}^{++}$and $\mathcal{O}_{m \mid \infty}^{++}$are conjectured to be equivalent.

1.3. Further relations to other works. The results of this paper in addition provide conceptual clarification of various results and empirical observations in the literature. Here are a few examples.

For a given positive integer $n$, let $\lambda=\left(\lambda_{-m}, \cdots, \lambda_{-1}, \lambda_{1}, \cdots, \lambda_{N}\right)$ be a partition with $\lambda_{1} \leq n$, and let $\lambda^{\natural}=\left(\lambda_{-m}, \cdots, \lambda_{-1}, \lambda_{1}^{\prime}, \cdots, \lambda_{n}^{\prime}\right)$, where $\left(\lambda_{1}^{\prime}, \cdots, \lambda_{n}^{\prime}\right)$ denotes the conjugate partition of $\left(\lambda_{1}, \cdots, \lambda_{N}\right)$. A classical result of Sergeev [Sv says that the irreducible $\mathfrak{g l}(m \mid n)$-modules with highest weights $\lambda^{\natural}$ associated to such partitions are exactly those appearing in the tensor powers of the natural module $\mathbb{C}^{m \mid n}$. Our duality implies that the character of the irreducible $\mathfrak{g l}(m \mid n)$-module $L_{n}\left(\lambda^{\natural}\right)$ is given by the hook Schur polynomials, which were obtained via different approaches in $\mathrm{Sv}$ and $\mathrm{BR}$.

For a given partition $\lambda$ as above, since the irreducible $\mathfrak{g l}(m+N)$-module $L_{N}(\lambda)$ admits the Weyl character formula, our duality results imply immediately that the irreducible $\mathfrak{g l}(m \mid n)$-module $L_{n}\left(\lambda^{\natural}\right)$ affords a Weyl-type character formula. This recovers and explains the results of $\mathrm{CZ}$.

Our results also provide a conceptual explanation of the similarity, observed in [LM], of the formulas loc. cit. with the ones in Br1] that the number of monomial basis elements appearing in a canonical basis is always a power of 2 . The combination of our stability and duality results explains when and why these two seemingly unrelated calculations yield the same results, and the truncation maps explain how to make sense of the difference when some terms are missing.

We believe that the duality principle between Lie algebras and Lie superalgebras, formulated and established here for type $A$, provides a new approach to the representation theory of Lie superalgebras and should be applicable to more general module categories and other types of Lie (super)algebras. It would also be interesting to extend this to the positive characteristic case (cf. $[\mathrm{Ku}]$ ). We intend to address these issues in sequels to this paper.

1.4. Organization. The paper is organized as follows. In Section 2 we set up the basics on (dual) canonical bases on Fock spaces. We introduce the truncation 
maps between Fock spaces and show that the bar involution commutes with the truncations. Section 3 addresses the representation theory of $\mathfrak{g l}(m \mid n)$ and $\mathfrak{g l}(m \mid \infty)$. In Section 4 we formulate and establish closed formulas for (dual) canonical bases on the Fock spaces $\varepsilon^{m+n}$ and $\widehat{\mathcal{E}}^{m+\infty}$. In Section 5 we reformulate a parabolic KL theory for the representations of $\mathfrak{g l}(m+n)$ in terms of the Fock space $\mathcal{E}^{m+n}$. In Section [6 we establish the isomorphism of the Fock spaces and the isomorphism of Grothendieck groups with favorable properties. Notation: $\mathbb{N}=\{0,1,2, \cdots\}$.

\section{Basic COnstructions of Fock SPACES}

2.1. Basics on quantum groups and the Iwahori-Hecke algebra. The quantum group $U_{q}(\mathfrak{g l}(\infty))$ is the $\mathbb{Q}(q)$-algebra generated by $E_{a}, F_{a}, K_{a}^{ \pm}, a \in \mathbb{Z}$, subject to the relations

$$
\begin{aligned}
K_{a} K_{a}^{-1}=K_{a}^{-1} K_{a}=1, & K_{a} K_{b}=K_{b} K_{a}, \\
K_{a} E_{b} K_{a}^{-1}=q^{\delta_{a, b}-\delta_{a, b+1} E_{b},} & K_{a} F_{b} K_{a}^{-1}=q^{\delta_{a, b+1}-\delta_{a, b}} F_{b}, \\
E_{a} F_{b}-F_{b} E_{a}= & \delta_{a, b}\left(K_{a, a+1}-K_{a+1, a}\right) /\left(q-q^{-1}\right), \\
E_{a} E_{b}=E_{b} E_{a}, & F_{a} F_{b}=F_{b} F_{a}, \quad \text { if }|a-b|>1, \\
E_{a}^{2} E_{b}+E_{b} E_{a}^{2}= & \left(q+q^{-1}\right) E_{a} E_{b} E_{a}, \quad \text { if }|a-b|=1, \\
F_{a}^{2} F_{b}+F_{b} F_{a}^{2}= & \left(q+q^{-1}\right) F_{a} F_{b} F_{a}, \quad \text { if }|a-b|=1 .
\end{aligned}
$$

Here $K_{a, a+1}:=K_{a} K_{a+1}^{-1}, a \in \mathbb{Z}$. Define the bar involution on $U_{q}(\mathfrak{g l}(\infty))$ to be the anti-linear automorphism ${ }^{-}: E_{a} \mapsto E_{a}, \quad F_{a} \mapsto F_{a}, \quad K_{a} \mapsto K_{a}^{-1}$. Here by anti-linear we mean the automorphism of $\mathbb{Q}(q)$ given by $q \mapsto q^{-1}$.

Let $\mathbb{V}$ be the natural $U_{q}(\mathfrak{g l}(\infty))$-module with basis $\left\{v_{a}\right\}_{a \in \mathbb{Z}}$ and $\mathbb{W}:=\mathbb{V}^{*}$ the dual module with basis $\left\{w_{a}\right\}_{a \in \mathbb{Z}}$ such that $w_{a}\left(v_{b}\right)=(-q)^{-a} \delta_{a, b}$. We have

$$
\begin{array}{r}
K_{a} v_{b}=q^{\delta_{a b}} v_{b}, \quad E_{a} v_{b}=\delta_{a+1, b} v_{a}, \quad F_{a} v_{b}=\delta_{a, b} v_{a+1}, \\
K_{a} w_{b}=q^{-\delta_{a b}} w_{b}, \quad E_{a} w_{b}=\delta_{a, b} w_{a+1}, \quad F_{a} w_{b}=\delta_{a+1, b} w_{a} .
\end{array}
$$

Following Br1] we shall use the co-multiplication $\Delta$ on $U_{q}(\mathfrak{g l}(\infty))$ defined by:

$$
\begin{aligned}
\Delta\left(E_{a}\right) & =1 \otimes E_{a}+E_{a} \otimes K_{a+1, a}, \\
\Delta\left(F_{a}\right) & =F_{a} \otimes 1+K_{a, a+1} \otimes F_{a}, \quad \Delta\left(K_{a}\right)=K_{a} \otimes K_{a} .
\end{aligned}
$$

We let $\mathcal{U}=U_{q}(\mathfrak{s l}(\infty))$ denote the subalgebra with generators $E_{a}, F_{a}, K_{a, a+1}, a \in \mathbb{Z}$.

For $m \in \mathbb{N}, n \in \mathbb{N} \cup \infty$, we let $I(m \mid n):=\{-m,-m+1, \cdots,-1\} \cup\{1,2, \cdots, n\}$. Denote by $S_{m+n}$ the symmetric group of (finite) permutations on $I(m \mid n)$, and $S_{m \mid n}$ its subgroup $S_{m} \times S_{n}$. Then $S_{m+n}$ is generated by the simple transpositions $s_{-m+1}=(-m,-m+1), \ldots, s_{-1}=(-2,-1), s_{0}=(-1,1), s_{1}=(1,2), \ldots, s_{n-1}=$ $(n-1, n)$, and $S_{m \mid n}$ is generated by those $s_{i}$ with $i \neq 0$.

The Iwahori-Hecke algebra $\mathcal{H}_{m+n}$ is the $\mathbb{Q}(q)$-algebra with generators $H_{i}$, where $-m+1 \leq i \leq n-1$, subject to the relations $\left(H_{i}-q^{-1}\right)\left(H_{i}+q\right)=0, H_{i} H_{i+1} H_{i}=$ $H_{i+1} H_{i} H_{i+1}, H_{i} H_{j}=H_{j} H_{i}$ for $|i-j|>1$. Associated to $x \in S_{m+n}$ with a reduced expression $x=s_{i_{1}} \cdots s_{i_{r}}$, we define $H_{x}:=H_{i_{1}} \cdots H_{i_{r}}$. The bar involution on $\mathcal{H}_{m+n}$ is the unique anti-linear automorphism defined by $\overline{H_{x}}=H_{x^{-1}}^{-1}$ for all $x \in S_{m+n}$. We denote by $\mathcal{H}_{m \mid n}$ the subalgebra generated by those $H_{i}$ with $i \neq 0$; that is, $\mathcal{H}_{m \mid n}$ is the Hecke algebra corresponding to $S_{m \mid n}$. Denote by $w_{0}$ the longest element in $S_{m \mid n}$, and let $\widehat{H}_{0}:=\sum_{x \in S_{m \mid n}}(-q)^{\ell(x)-\ell\left(w_{0}\right)} H_{x}$. 
2.2. The spaces $\Lambda^{\infty} \mathbb{V}, \mathcal{E}^{m+n}$ and $\varepsilon^{m+\infty}$. Let $P$ be the free abelian group with basis $\left\{\epsilon_{a} \mid a \in \mathbb{Z}\right\}$ equipped with a bilinear form $(\cdot \mid \cdot)$ for which the $\epsilon_{a}$ 's are orthonormal. We define a partial order on $P$ by declaring $\nu \geq \mu$ for $\nu, \mu \in P$ if $\nu-\mu$ is a non-negative integral linear combination of $\epsilon_{a}-\epsilon_{a+1}, a \in \mathbb{Z}$. For $n \in \mathbb{N} \cup \infty$, we let $\mathbb{Z}^{m+n}$ or $\mathbb{Z}^{m \mid n}$ be the set of integer-valued functions on $I(m \mid n)$. Define

$$
\mathrm{wt}^{\epsilon}(f):=\sum_{i \in I(m \mid n)} \epsilon_{f(i)}, \quad \text { for } f \in \mathbb{Z}^{m+n} .
$$

For a finite $n$ consider $\mathbb{T}^{m+n}:=\mathbb{V}^{\otimes(m+n)}$, where we adopt the convention that the $m+n$ tensor factors are indexed by $I(m \mid n)$. Similar conventions will apply to similar situations below. For $f \in \mathbb{Z}^{m+n}$, let

$$
\mathcal{V}_{f}:=v_{f(-m)} \otimes \cdots \otimes v_{f(-1)} \otimes v_{f(1)} \otimes \cdots \otimes v_{f(n)} .
$$

Let $\mathcal{H}_{m+n}$ act on $\mathbb{T}^{m+n}$ on the right by:

$$
\mathcal{V}_{f} H_{i}= \begin{cases}\mathcal{V}_{f \cdot s_{i}}, & \text { if } f<f \cdot s_{i}, \\ q^{-1} \mathcal{V}_{f}, & \text { if } f=f \cdot s_{i}, \\ \mathcal{V}_{f \cdot s_{i}}-\left(q-q^{-1}\right) \mathcal{V}_{f}, & \text { if } f>f \cdot s_{i} .\end{cases}
$$

The Bruhat ordering $\leq$ on $\mathbb{Z}^{m+n}$ comes from the Bruhat ordering on $S_{m+n}$, which is the transitive closure of the relation $f<f \cdot \tau_{i j}$ if $f(i)<f(j)$, for $i, j \in I(m \mid n)$ with $i<j$. Here and further on, $\tau_{i j}$ denotes the transposition interchanging $i, j$. The algebra $\mathcal{U}$ acts on $\mathbb{T}^{m+n}$ via the co-multiplication $\Delta$.

Lemma 2.1 ([Jim). The actions of $\mathcal{U}$ and $\mathcal{H}_{m+n}$ on $\mathbb{T}^{m+n}$ commute with each other.

Different commuting actions of $\mathcal{U}$ and the Hecke algebra on a tensor power of $\mathbb{V}$ were used in $\overline{\mathrm{KMS}}$ to construct the space $\Lambda^{n} \mathbb{V}$ of finite $q$-wedges and then the space of infinite-wedges by taking the limit $n \rightarrow \infty$ appropriately. These spaces carry the action of quantum affine algebras as well as the action of $\mathcal{U}$ (as a limiting case). The constructions in loc. cit. carry over using the above actions of $\mathcal{U}$ and the Hecke algebra as formulated below, and we refer to loc. cit. for details.

One can think of $\Lambda^{n} \mathbb{V}$ either as the subspace $i m \widehat{H}_{0}$ or the quotient $\mathbb{T}^{n} / \operatorname{ker} \widehat{H}_{0}$ of $\mathbb{T}^{n}$. Here ker $\widehat{H}_{0}$ equals the sum of the kernels of the operators $H_{i}-q^{-1}, 1 \leq i \leq n-1$ (note that the Hecke algebra generator $T_{i}$ used in loc. cit. corresponds to our $-q H_{i}$ ). The $q$-wedge $v_{a_{1}} \wedge \cdots \wedge v_{a_{n}}$ is an element of $\Lambda^{n} \mathbb{V}$, which is the image of $v_{a_{1}} \otimes \cdots \otimes v_{a_{n}}$ under the canonical map when $\Lambda^{n} \mathbb{V}$ is regarded as the quotient space $\mathbb{T}^{n} / \operatorname{ker} \widehat{H}_{0}$. We have

$$
\cdots \wedge v_{a_{i}} \wedge v_{a_{i+1}} \wedge \cdots=-q^{-1}\left(\cdots \wedge v_{a_{i+1}} \wedge v_{a_{i}} \wedge \cdots\right), \quad \text { if } a_{i}<a_{i+1}
$$

It follows that the elements $v_{a_{1}} \wedge \cdots \wedge v_{a_{n}}$, where $a_{1}>\cdots>a_{n}$, form a basis for $\Lambda^{n} \mathbb{V}$. By Lemma 2.1. $\mathcal{U}$ acts naturally on $\Lambda^{n} \mathbb{V}$.

By taking $n \rightarrow \infty$, one defines $\Lambda^{\infty} \mathbb{V}$ with a $\mathcal{U}$-action, with a basis given by the infinite $q$-wedges $v_{m_{1}} \wedge v_{m_{2}} \wedge v_{m_{3}} \wedge \cdots$, where $m_{1}>m_{2}>m_{3}>\cdots$, and $m_{i}=1-i$ for $i \gg 0$ (our $\Lambda^{\infty} \mathbb{V}$ is $F_{(0)}$ in [KMS]). Alternatively, $\Lambda^{\infty} \mathbb{V}$ has a basis

$$
|\lambda\rangle:=v_{\lambda_{1}} \wedge v_{\lambda_{2}-1} \wedge v_{\lambda_{3}-2} \wedge \cdots
$$


where $\lambda=\left(\lambda_{1}, \lambda_{2}, \cdots\right)$ runs over the set of all partitions. Set (for a finite $n$ )

$$
\begin{aligned}
\mathbb{Z}_{+}^{m+n}:= & \left\{f \in \mathbb{Z}^{m+n} \mid f(-m)>\cdots>f(-1), f(1)>\cdots>f(n)\right\}, \\
\mathbb{Z}_{++}^{m+n}:= & \left\{f \in \mathbb{Z}_{+}^{m+n} \mid f(n) \geq 1-n\right\}, \\
\mathbb{Z}_{+}^{m+\infty}:= & \left\{f \in \mathbb{Z}^{m+\infty} \mid f(-m)>\cdots>f(-1),\right. \\
\quad & \quad f(1)>f(2)>\cdots ; f(i)=1-i \text { for } i \gg 0\} .
\end{aligned}
$$

We will call an element in $\mathbb{Z}_{+}^{m+n}$ or $\mathbb{Z}_{+}^{m+\infty}$ dominant.

For $n \in \mathbb{N} \cup \infty$, we let

$$
\varepsilon^{m+n}:=\Lambda^{m} \mathbb{V} \otimes \Lambda^{n} \mathbb{V},
$$

where the factors are indexed by $I(m \mid n)$. It has the monomial basis

$$
\mathcal{K}_{f}:= \begin{cases}v_{f(-m)} \wedge \cdots \wedge v_{f(-1)} \otimes v_{f(1)} \wedge \cdots \wedge v_{f(n)}, & \text { for finite } n, \\ v_{f(-m)} \wedge \cdots \wedge v_{f(-1)} \otimes v_{f(1)} \wedge v_{f(2)} \wedge \cdots, & \text { for } n=\infty,\end{cases}
$$

where $f$ runs over the set $\mathbb{Z}_{+}^{m+n}$. Recalling $\mathbb{T}^{m+n}=\operatorname{ker} \widehat{H}_{0} \oplus \operatorname{Im} \widehat{H}_{0}$, cf. [KMS], we may regard equivalently $\mathcal{E}^{m+n}$ as the subspace $\operatorname{Im} \widehat{H}_{0}$ of $\mathbb{T}^{m+n}$ for $n$ finite.

Let $g \in \mathbb{Z}_{+}^{m+0}$ or $g \in \mathbb{Z}_{+}^{0+n}$. For $a \in \mathbb{Z}$ define $\tilde{e}_{a, a+1} g$ (respectively $\tilde{e}_{a, a-1} g$ ) to be the strictly decreasing sequence of integers obtained from $g$ by replacing the value that is $a+1$ (respectively $a-1$ ) by $a$, if $a+1$ (respectively $a-1$ ) appears in $g$ and $a$ does not appear in $g$. Otherwise set $\tilde{e}_{a, a \pm 1} g=\emptyset$. For $g \in \mathbb{Z}^{m+n}$ we let $g<0$ and $g^{>0}$ denote the restrictions of $g$ to $\{-m, \cdots,-1\}$ and $\{1, \cdots, n\}$, respectively, and write $g=\left(g^{<0} \mid g^{>0}\right)$. Now for $m, n>0$ let $g \in \mathbb{Z}_{+}^{m+n}$. We set $\mathcal{K}_{g}=0$ if $g$ is of the form $\left(g^{<0} \mid \emptyset\right)$ or $\left(\emptyset \mid g^{>0}\right)$. The formulas for $\Delta$ on $\Lambda^{m} \mathbb{V} \otimes \Lambda^{n} \mathbb{V}$ and the straightening relations (2.3) give us the following formula.

Lemma 2.2. Let $n \in \mathbb{N} \cup \infty$. For $f=\left(f^{<0} \mid f^{>0}\right) \in \mathbb{Z}_{+}^{m+n}, \mathcal{U}=U_{q}(\mathfrak{s l}(\infty))$ acts on $\varepsilon^{m+n}$ as follows:

$$
\begin{aligned}
& E_{a}\left(\mathcal{K}_{(f<0 \mid f>0)}\right)=\mathcal{K}_{\left(f<0 \mid \tilde{e}_{a, a+1} f>0\right)}+q^{-\left(w t^{\epsilon}\left(f^{>0}\right) \mid \epsilon_{a}\right)+\left(w t^{\epsilon}\left(f^{>0}\right) \mid \epsilon_{a+1}\right)} \mathcal{K}_{\left(\tilde{e}_{a, a+1} f^{<0} \mid f>0\right)}, \\
& F_{a}\left(\mathcal{K}_{\left(f^{<0} \mid f>0\right)}\right)=\mathcal{K}_{\left(\tilde{e}_{a+1, a} f<0 \mid f>0\right)}+q^{\left(w t^{\epsilon}\left(f^{<0}\right) \mid \epsilon_{a}\right)-\left(w t^{\epsilon}\left(f^{<0}\right) \mid \epsilon_{a+1}\right)} \mathcal{K}_{\left(f^{<0} \mid \tilde{e}_{a+1, a} f>0\right)} .
\end{aligned}
$$

2.3. Super Bruhat ordering on $\mathbb{Z}^{m \mid n}$. Let $n \in \mathbb{N} \cup \infty$. For $i \in I(m \mid n)$ we define $d_{i} \in \mathbb{Z}^{m \mid n}$ by $j \mapsto-\operatorname{sgn}(i) \delta_{i j}$. For $f, g \in \mathbb{Z}^{m \mid n}$, we write $f \downarrow g$ if one of the following holds:

(1) $g=f-d_{i}+d_{j}$ for some $i<0<j$ such that $f(i)=f(j)$;

(2) $g=f \cdot \tau_{i j}$ for some $i<j<0$ such that $f(i)>f(j)$;

(3) $g=f \cdot \tau_{i j}$ for some $0<i<j$ such that $f(i)<f(j)$.

The super Bruhat ordering on $\mathbb{Z}^{m \mid n}$ is defined as follows: for $f, g \in \mathbb{Z}^{m \mid n}$, we say that $f \succ g$ if there exists a sequence $f=h_{1}, \ldots, h_{r}=g \in \mathbb{Z}^{m \mid n}$ such that $h_{1} \downarrow h_{2}, \cdots, h_{r-1} \downarrow h_{r}$. It can also be described (cf. [Br1]) in terms of the $\epsilon$ weights, which are defined by:

$$
\mathrm{wt}^{\epsilon}(f):=\sum_{i \in I(m \mid n)}-\operatorname{sgn}(i) \epsilon_{f(i)}, \quad \text { for } f \in \mathbb{Z}^{m \mid n} .
$$

The super Bruhat ordering is defined to be compatible with the one on the set of integral weights for $\mathfrak{g l}(m \mid n)$. Here and further the superscript Br stands for 
Brundan's version Br1. Our weight $f$ corresponds to $f^{B r}=-f$. Our $d_{i}$ here differs from the one in [Br1] by a sign. Set (for a finite $n$ )

$$
\begin{aligned}
& \mathbb{Z}_{+}^{m \mid n}:=\left\{f \in \mathbb{Z}^{m \mid n} \mid f(-m)>\cdots>f(-1), f(1)<\cdots<f(n)\right\}, \\
& \mathbb{Z}_{++}^{m \mid n}:=\left\{f \in \mathbb{Z}_{+}^{m \mid n} \mid f(n) \leq n\right\}, \\
& \mathbb{Z}_{+}^{m \mid \infty}:=\left\{f \in \mathbb{Z}^{m \mid \infty} \mid f(-m)>\cdots>f(-1),\right. \\
&\quad f(1)<f(2)<\cdots ; f(i)=i \text { for } i \gg 0\} .
\end{aligned}
$$

An element in $\mathbb{Z}_{+}^{m \mid n}$ is called a dominant weight. (Note that $\mathbb{Z}_{+}^{m \mid n}=-\mathbb{Z}_{+}^{m \mid n, B r}$.) For $n \in \mathbb{N} \cup \infty$ and $f \in \mathbb{Z}^{m \mid n}$ conjugate under the action of $S_{m \mid n}$ to an element in $\mathbb{Z}_{+}^{m \mid n}$, define the degree of atypicality of $f$ to be the

$$
\# f:=|\{f(-m), \cdots, f(-1)\} \cap\{f(1), f(2), \cdots\}| .
$$

If $f, g \in \mathbb{Z}_{+}^{m \mid n}$ are comparable under the super Bruhat ordering, then $\# f=\# g$. If $\# f=0$, we say that $f$ is typical. For later use we introduce the following.

Definition 2.3. Let $f \in \mathbb{Z}_{+}^{m \mid k}$ (respectively $f \in \mathbb{Z}_{+}^{m+k}$ ), with $k \in \mathbb{N} \cup \infty, n \leq k$. Define $f^{(n)} \in \mathbb{Z}_{+}^{m \mid n}$ (respectively $f^{(n)} \in \mathbb{Z}_{+}^{m+n}$ ) to be the restriction of $f$ to $I(m \mid n)$. We say that $n$ is sufficiently large for $f$ if $\# f=\# f^{(n)}$ and $f\left(n^{\prime}\right)=n^{\prime}$ (respectively $\left.f\left(n^{\prime}\right)=1-n^{\prime}\right)$ for $n^{\prime} \geq n+1$. When $f$ is clear from the context we shall simply write $n \gg 0$.

2.4. The spaces $\Lambda^{\infty} \mathbb{V}^{*}, \mathcal{E}^{m \mid n}$ and $\mathcal{E}^{m \mid \infty}$. Recall that $\mathbb{W}=\mathbb{V}^{*}$ is the dual module of $\mathcal{U}$ with basis $\left\{w_{a}\right\}_{a \in \mathbb{Z}}$. The space $\mathbb{T}^{m \mid n}:=\mathbb{V}^{\otimes m} \otimes \mathbb{W}^{\otimes n}$ has the monomial basis $M_{f}:=v_{f(-m)} \otimes \cdots \otimes v_{f(-1)} \otimes w_{f(1)} \otimes \cdots \otimes w_{f(n)}, f \in \mathbb{Z}^{m \mid n}$. Let $\mathcal{H}_{m \mid n}$ act on $\mathbb{T}^{m \mid n}$ on the right (cf. [Br1]) by:

$$
M_{f} H_{i}= \begin{cases}M_{f \cdot s_{i}}, & \text { if } f \prec f \cdot s_{i}, \\ q^{-1} M_{f}, & \text { if } f=f \cdot s_{i}, \\ M_{f \cdot s_{i}}-\left(q-q^{-1}\right) M_{f}, & \text { if } f \succ f \cdot s_{i} .\end{cases}
$$

The algebra $\mathcal{U}$ acts on $\mathbb{T}^{m \mid n}$ via the co-multiplication $\Delta$.

Lemma 2.4. The actions of $\mathcal{U}$ and $\mathcal{H}_{m \mid n}$ on $\mathbb{T}^{m \mid n}$ commute with each other.

For a finite $n$, we can again define $\mathcal{E}^{m \mid n}$ to be either the image of the operator $\widehat{H}_{0}$ in $\mathbb{T}^{m \mid n}$, or the quotient of $\mathbb{T}^{m \mid n}$ by the kernel of $\widehat{H}_{0}$. The following straightening relations hold in $\mathcal{E}^{m \mid n}$ (note the conditions $a_{i-1}<a_{i}$ and $b_{i}>b_{i+1}$ reflect the super Bruhat order):

$$
\begin{aligned}
& \cdots \wedge v_{a_{i-1}} \wedge v_{a_{i}} \wedge \cdots=-q^{-1}\left(\cdots v_{a_{i}} \wedge v_{a_{i-1}} \wedge \cdots\right), \quad \text { if } a_{i-1}<a_{i}, i<0 \\
& \cdots \wedge w_{b_{i}} \wedge w_{b_{i+1}} \wedge \cdots=-q^{-1}\left(\cdots w_{b_{i+1}} \wedge w_{b_{i}} \wedge \cdots\right), \text { if } b_{i}>b_{i+1}, i>0 .
\end{aligned}
$$

We can repeat the procedure in $\mathrm{KMS}$ to construct the space $\Lambda^{\infty} \mathbb{W}$ of semiinfinite $q$-wedges $w_{n_{1}} \wedge w_{n_{2}} \wedge \cdots$, where $n_{i}=i$ for $i \gg 0$, which carries a $\mathcal{U}$-module structure. Writing the conjugate partition of $\lambda$ as $\lambda^{\prime}=\left(\lambda_{1}^{\prime}, \lambda_{2}^{\prime}, \cdots\right)$, we set

$$
\left|\lambda_{*}^{\prime}\right\rangle:=w_{1-\lambda_{1}^{\prime}} \wedge w_{2-\lambda_{2}^{\prime}} \wedge w_{3-\lambda_{3}^{\prime}} \wedge \cdots .
$$

The set $\left\{\left|\lambda_{*}^{\prime}\right\rangle\right\}$ provides another basis for $\Lambda^{\infty} \mathbb{W}$. Note that for a finite $n$, we have $\mathcal{E}^{m \mid n}=\Lambda^{m} \mathbb{V} \otimes \Lambda^{n} \mathbb{W}$ as $\mathcal{U}$-modules. Denote

$$
\mathcal{E}^{m \mid \infty}:=\Lambda^{m} \mathbb{V} \otimes \Lambda^{\infty} \mathbb{W},
$$


which is a $\mathcal{U}$-module via $\Delta . \mathcal{E}^{m \mid n}$ has the monomial basis

$$
K_{f}:= \begin{cases}v_{f(-m)} \wedge \cdots \wedge v_{f(-1)} \otimes w_{f(1)} \wedge \cdots \wedge w_{f(n)}, & \text { for finite } n, \\ v_{f(-m)} \wedge \cdots \wedge v_{f(-1)} \otimes w_{f(1)} \wedge w_{f(2)} \wedge \cdots, & \text { for } n=\infty,\end{cases}
$$

where $f$ runs over $\mathbb{Z}_{+}^{m \mid n}$.

2.5. Bases for $\widehat{\mathcal{E}}^{m \mid n}$ and $\widehat{\mathcal{\varepsilon}}^{m \mid \infty}$. Let $n \in \mathbb{N} \cup \infty$. For $d \in \mathbb{N}$ let $\mathcal{E}_{\geq-d}^{m \mid n}$ be the $\mathbb{Q}(q)$-subspace of $\mathcal{E}^{m \mid n}$ spanned by $K_{f}$ with $f(i) \geq-d$, for all $i \in\{1, \cdots, n\}$. We shall denote a certain topological completion of $\mathcal{E}^{m \mid n}$ by $\widehat{\mathcal{E}}^{m \mid n}$ whose elements may be viewed as infinite $\mathbb{Q}(q)$-linear combinations of elements in $\mathcal{E}^{m \mid n}$, which under the projection onto $\varepsilon_{\geq-d}^{m \mid n}$ are finite sums for all $d \in \mathbb{N}$ (cf. [Br1, $\S 2$-d]). The following proposition for a finite $n$ is Theorem 3.5 [Br1], which is similar to results of Lusztig Lu2]. The proof of the $n=\infty$ case is similar and in a sense even simpler, since $\Lambda^{\infty} \mathbb{V}^{*}$ is a highest weight $\mathcal{U}$-module.

Proposition 2.5. Let $n \in \mathbb{N} \cup \infty$. There exists a unique continuous, anti-linear bar map ${ }^{-}: \widehat{\mathcal{E}}^{m \mid n} \rightarrow \widehat{\mathcal{E}}^{m \mid n}$ such that

(1) $\overline{K_{f}}=K_{f}$ for all typical $f \in \mathbb{Z}_{+}^{m \mid n}$.

(2) $\overline{X u}=\bar{X} \bar{u}$ for all $X \in \mathcal{U}$ and $u \in \widehat{\mathcal{E}}^{m \mid n}$.

(3) The bar map is an involution.

(4) $\overline{K_{f}}=K_{f}+(*)$ where $(*)$ is a (possibly infinite) $\mathbb{Z}\left[q, q^{-1}\right]$-linear combination of $K_{g}$ 's for $g \in \mathbb{Z}_{+}^{m \mid n}$ with $g \prec f$.

The next theorem now follows by standard arguments (cf. [KL, Lu2, Du, Br1]).

Theorem 2.6. Let $n \in \mathbb{N} \cup \infty$. There exist a unique canonical basis $\left\{U_{f}\right\}$ and a unique dual canonical basis $\left\{L_{f}\right\}$, where $f \in \mathbb{Z}_{+}^{m \mid n}$, for $\widehat{\mathcal{E}}^{m \mid n}$ such that

(1) $\bar{U}_{f}=U_{f}$ and $\bar{L}_{f}=L_{f}$,

(2) $U_{f} \in K_{f}+\widehat{\sum}_{g \in \mathbb{Z}_{+}^{m \mid n}} q \mathbb{Z}[q] K_{g}$ and $L_{f} \in K_{f}+\widehat{\sum}_{g \in \mathbb{Z}_{+}^{m \mid n}} q^{-1} \mathbb{Z}\left[q^{-1}\right] K_{g}$,

(3) $U_{f}=K_{f}+(*)$ and $L_{f}=K_{f}+(* *)$ where $(*)$ and $(* *)$ are (possibly infinite) $\mathbb{Z}\left[q, q^{-1}\right]$-linear combinations of $K_{g}$ 's for $g \in \mathbb{Z}_{+}^{m \mid n}$ such that $g \prec f$.

Let $n \in \mathbb{N} \cup \infty$. Following Br1, we define the Kazhdan-Lusztig polynomials $u_{g, f}(q) \in \mathbb{Z}[q], \ell_{g, f}(q) \in \mathbb{Z}\left[q^{-1}\right]$ associated to $f, g \in \mathbb{Z}_{+}^{m \mid n}$ by

$$
U_{f}=\sum_{g \in \mathbb{Z}_{+}^{m \mid n}} u_{g, f}(q) K_{g}, \quad L_{f}=\sum_{g \in \mathbb{Z}_{+}^{m \mid n}} \ell_{g, f}(q) K_{g} .
$$

Note that $u_{g, f}(q)=\ell_{g, f}(q)=0$ unless $g \preccurlyeq f, u_{f, f}(q)=\ell_{f, f}(q)=1$, and $u_{g, f}(q) \in$ $q \mathbb{Z}[q], \ell_{g, f}(q) \in q^{-1} \mathbb{Z}\left[q^{-1}\right]$ for $g \neq f$.

2.6. The truncation map. Let $n$ be finite. Denote by $\mathcal{E}_{+}^{m \mid n}$ the subspace of $\mathcal{E}^{m \mid n}$ spanned by $K_{f}$ for $f \in \mathbb{Z}_{++}^{m \mid n}$. For $n>0$, define the subalgebra $U_{q}\left(\mathfrak{s l} l_{\leq n}\right)$ to be the subalgebra of $U_{q}(\mathfrak{s l}(\infty))$ generated by $E_{a-1}, F_{a-1}$ and $K_{a-1, a}$, with $a \leq n$.

Lemma 2.7. $\mathcal{E}_{+}^{m \mid n}$ is a bar-invariant subspace of $\mathcal{E}^{m \mid n}$, and it is a $U_{q}\left(\mathfrak{s l}_{\leq n}\right)$-module.

Proof. The first half follows from Proposition 2.5 (4), and the second half is an easy consequence of Lemma 2.2 
We define the truncation map to be the linear map $\mathfrak{T r}_{n+1, n}: \widehat{\mathcal{E}}_{+}^{m \mid n+1} \longrightarrow \widehat{\mathcal{E}}_{+}^{m \mid n}$ which sends $K_{f}$ to $K_{f(n)}$ if $f(n+1)=n+1$, and to 0 otherwise. For $n^{\prime}>n$, we define the truncation map $\mathcal{T r}_{n^{\prime}, n}: \widehat{\mathcal{E}}_{+}^{m \mid n^{\prime}} \longrightarrow \widehat{\mathcal{E}}_{+}^{m \mid n}$ to be $\mathfrak{T r}_{n^{\prime}, n}:=\mathfrak{T r}_{n+1, n} \circ \cdots \circ \mathfrak{T r}_{n^{\prime}, n^{\prime}-1}$. Similarly, the truncation map $\mathfrak{T r}_{n}: \widehat{\mathcal{E}}^{m \mid \infty} \longrightarrow \widehat{\mathcal{E}}_{+}^{m \mid n}$ is defined by sending $K_{f}$ to $K_{f^{(n)}}$ if $f=\left(f^{(n)}, n+1, n+2, \cdots\right)$, and to 0 otherwise.

Proposition 2.8. The truncation map $\mathfrak{T r}_{n+1, n}: \widehat{\mathcal{E}}_{+}^{m \mid n+1} \rightarrow \widehat{\mathcal{E}}_{+}^{m \mid n}$ commutes with the bar-involution.

Proof. Throughout the proof $\mathcal{E}^{m \mid n}$ will be regarded as a subspace of $\mathbb{T}^{m \mid n}$ for all $m$ and $n$. Let $\widehat{\mathbb{T}}^{m \mid n, 1}$ be a topological completion of $\mathcal{E}^{m \mid n} \otimes \mathbb{W}$ defined in an analogous way as the completion $\widehat{\mathcal{E}}^{m \mid n}$ in Subsection 2.5. Thus $\widehat{\mathbb{T}}^{m \mid n, 1}$ contains $\widehat{\mathcal{E}}^{m \mid n+1}$ as a subspace. Now $K_{f(n)} \otimes w_{f(n+1)}$ with $f^{(n)} \in \mathbb{Z}_{+}^{m \mid n}, f(n+1) \in \mathbb{Z}$, form a basis of $\widehat{\mathbb{T}}^{m \mid n, 1}$. One can follow Lusztig (Section 24.1.1 in [Lu2]) to define a bar-involution on $\widehat{\mathbb{T}}^{m \mid n, 1}$ using a quasi $R$-matrix $\Theta^{(m \mid n+1)}$ such that

$$
\overline{K_{f^{(n)}} \otimes w_{f(n+1)}}:=\Theta^{(m \mid n+1)}\left(\overline{K_{f^{(n)}}} \otimes w_{f(n+1)}\right) .
$$

Let $X_{a b}$ be endomorphisms of $\mathbb{W}$ such that $X_{a b} w_{c}=\delta_{a c} w_{b}, a, b, c \in \mathbb{Z}$. By exploiting the structure of the quasi $R$-matrix of $\mathcal{U}$ (Theorem 8.1 in $\overline{\mathrm{KT}}$, and Theorem 3 in $[\mathrm{KR}]$ ), we can show that there exist elements $\hat{E}_{a b}$ in $\mathcal{U}$ constructed from $E_{c, c+1}$ and $K_{c}^{ \pm 1}$ only and with the property $K_{c} \hat{E}_{a b} K_{c}^{-1}=q^{\delta_{a c}-\delta_{b c}} \hat{E}_{a b}$ such that

$$
\Theta^{(m \mid n+1)}=1 \otimes 1+\left(q-q^{-1}\right) \sum_{a<b} \hat{E}_{a b} \otimes X_{b a} .
$$

This is a variant of Jimbo's result Jim on the $R$-matrix in the tensor product of an arbitrary representation with the natural representation of $U_{q}(\mathfrak{g l}(k))$ for finite $k$. We have

$$
\begin{aligned}
\overline{K_{f(n)} \otimes w_{f(n+1)}}= & \overline{K_{f(n)}} \otimes w_{f(n+1)} \\
& +\left(q-q^{-1}\right) \sum_{a<f(n+1)} \hat{E}_{a f(n+1)} \overline{K_{f(n)}} \otimes w_{a} .
\end{aligned}
$$

Denote by $\widehat{\mathbb{T}}_{+}^{m \mid n, 1}$ the subspace of $\widehat{\mathbb{T}}^{m \mid n, 1}$ spanned by all $K_{f(n)} \otimes w_{f(n+1)}$ with $f^{(n)} \in \mathbb{Z}_{+}^{m \mid n}$ and $f(n+1) \leq n+1$. By (2.8), $\widehat{\mathbb{T}}_{+}^{m \mid n, 1}$ is invariant under the barinvolution. Introduce the following linear map:

$$
\check{\mathfrak{T}}_{n+1, n}: \widehat{\mathbb{T}}_{+}^{m \mid n, 1} \rightarrow \widehat{\mathcal{E}}^{m \mid n}, \quad K_{f^{(n)}} \otimes w_{f(n+1)} \mapsto \delta_{f(n+1), n+1} K_{f^{(n)}} .
$$

Applying it to (2.8) we see that $\check{\mathfrak{T}}_{n+1, n}$ commutes with the bar-involution.

Now $\widehat{\mathcal{E}}_{+}^{m \mid n+1}=\widehat{\mathcal{E}}^{m \mid n+1} \cap \widehat{\mathbb{T}}_{+}^{m \mid n, 1}$ and hence $\widehat{\mathcal{E}}_{+}^{m \mid n+1}$ is bar-invariant. The proposition follows since the restriction of $\check{\mathfrak{T}}_{n+1, n}$ to $\widehat{\mathcal{E}}_{+}^{m \mid n+1}$ coincides with $\mathfrak{T r}_{n+1, n}$.

Remark 2.9. It follows from Proposition 2.8 that $\mathfrak{T r}_{n^{\prime}, n}: \widehat{\mathcal{E}}_{+}^{m \mid n^{\prime}} \rightarrow \widehat{\mathcal{E}}_{+}^{m \mid n}$ commutes with the bar-involution for $n \leq n^{\prime} \leq \infty$.

Corollary 2.10. (1) $\left\{U_{f}\right\}_{f \in \mathbb{Z}_{++}^{m \mid n}}$ (respectively $\left\{L_{f}\right\}_{f \in \mathbb{Z}_{++}^{m \mid n}}$ ) is a basis for $\widehat{\mathcal{E}}_{+}^{m \mid n}$.

(2) $\mathfrak{T r}_{n+1, n}$ sends $U_{f} \in \widehat{\mathcal{E}}_{+}^{m \mid n+1}$ to $U_{f(n)}$ if $f(n+1)=n+1$, and to 0 otherwise.

(3) $\mathfrak{T r}_{n+1, n}$ sends $L_{f} \in \widehat{\mathcal{E}}_{+}^{m \mid n+1}$ to $L_{f^{(n)}}$ if $f(n+1)=n+1$, and to 0 otherwise. 
(4) For $f, g \in \mathbb{Z}_{++}^{m \mid n+1}$ such that $f(n+1)=g(n+1)=n+1$, we have

$$
u_{g, f}(q)=u_{g^{(n)}, f^{(n)}}(q), \quad \ell_{g, f}(q)=\ell_{g^{(n)}, f^{(n)}}(q) .
$$

Remark 2.11. For $n^{\prime}>n$ the map $\mathfrak{T r}_{n^{\prime}, n}$ is a $U_{q}\left(\mathfrak{s l}_{\leq n}\right)$-module homomorphism. Now for $f \in \mathbb{Z}_{+}^{m \mid \infty}$, Procedure 3.20 in Br1] is the same for all $f^{(n)}$ with $n \gg 0$, in the sense that it involves the same Chevalley generators and the same sequence of weights. The Chevalley generators lie in $U_{q}\left(\mathfrak{s} l_{\leq n_{0}}\right)$, for some fixed $n_{0} \gg 0$. This together with Corollary 2.10 implies that Procedure 3.20 in Br1 remains valid for $n=\infty$ as well.

2.7. The transition matrices. Let $n \in \mathbb{N}$ and $f \in \mathbb{Z}^{m \mid n}$ be $S_{m \mid n}$-conjugate to an element in $\mathbb{Z}_{+}^{m \mid n}$. For $-m \leq i<0<j \leq n$ with $f(i)=f(j)$, define Br1]

$$
\mathrm{L}_{i, j}(f):=f-a\left(d_{i}-d_{j}\right),
$$

where $a$ is the smallest positive integer such that $f-a\left(d_{i}-d_{j}\right)$ and all $\mathrm{L}_{k, l}(f)-$ $a\left(d_{i}-d_{j}\right)$ for $-m \leq i<k<0<l<j \leq n$ with $f(k)=f(l)$ are $S_{m \mid n}$-conjugate to elements of $\mathbb{Z}_{+}^{m \mid n}$. Furthermore define JZ

$$
\mathrm{R}_{i, j}(f):=f+b\left(d_{i}-d_{j}\right),
$$

where $b$ is the smallest positive integer such that $f+b\left(d_{i}-d_{j}\right)$ and all $\mathrm{R}_{k, l}(f)+$ $b\left(d_{i}-d_{j}\right)$ for $-m \leq k<i<0<j<l \leq n$ with $f(k)=f(l)$ are $S_{m \mid n}$-conjugate to elements of $\mathbb{Z}_{+}^{m \mid n}$.

Now let $f \in \mathbb{Z}_{+}^{m \mid n}$ and suppose \#f=k. Let $-m \leq i_{i}<i_{2}<\cdots<i_{k} \leq-1$ and $1 \leq j_{k}<j_{k-1}<\cdots<j_{1} \leq n$ be such that $f\left(i_{l}\right)=f\left(j_{l}\right)$, for $l=1, \cdots, k$. For a $k$-tuple $\theta=\left(\theta_{1}, \cdots, \theta_{k}\right) \in \mathbb{N}^{k}$ we define Br1]

$$
\begin{aligned}
& \mathrm{L}_{\theta}(f)=\left(\mathrm{L}_{i_{k}, j_{k}}^{\theta_{k}} \circ \cdots \circ \mathrm{L}_{i_{1}, j_{1}}^{\theta_{1}}(f)\right)^{+}, \quad \mathrm{L}_{\theta}^{\prime}(f)=\left(\mathrm{L}_{i_{1}, j_{1}}^{\theta_{1}} \circ \cdots \circ \mathrm{L}_{i_{k}, j_{k}}^{\theta_{k}}(f)\right)^{+}, \\
& \mathrm{R}_{\theta}(f)=\left(\mathrm{R}_{i_{1}, j_{1}}^{\theta_{1}} \circ \cdots \circ \mathrm{R}_{i_{k}, j_{k}}^{\theta_{k}}(f)\right)^{+}, \quad \mathrm{R}_{\theta}^{\prime}(f)=\left(\mathrm{R}_{i_{k}, j_{k}}^{\theta_{k}} \circ \cdots \circ \mathrm{R}_{i_{1}, j_{1}}^{\theta_{1}}(f)\right)^{+},
\end{aligned}
$$

where the superscript + here and further on stands for the unique $S_{m \mid n}$-conjugate in $\mathbb{Z}_{+}^{m \mid n}$. We shall denote $\mathrm{L}_{(1,1, \cdots, 1)}(f)$ and $\mathrm{R}_{(1,1, \cdots, 1)}(f)$ by $\mathrm{L}(f)$ and $\mathrm{R}(f)$, or sometimes by $f^{\mathrm{L}}$ and $f^{\mathrm{R}}$, respectively.

The corresponding operators $\mathrm{L}_{\theta}$ and $\mathrm{L}_{\theta}^{\prime}$ on $\mathbb{Z}_{+}^{m \mid \infty}$ are defined analogously, but it takes extra care to make sense of the R operators. Given $f, g \in \mathbb{Z}_{+}^{m \mid \infty}$ and $\theta \in \mathbb{N} \# f$, we say $\mathrm{R}_{\theta}(g)=f$ if there exists $n \gg 0$ (for $f$ and $g$ ) so that $\mathrm{R}_{\theta}\left(g^{(n)}\right)=f^{(n)}$. (Note the subtle point that $\mathrm{R}_{\theta}(g)$ is not defined for every $g \in \mathbb{Z}_{+}^{m \mid \infty}$.)

The next two lemmas follow from the definitions.

Lemma 2.12. Let $f=\left(f^{(n)}, n+1\right) \in \mathbb{Z}_{++}^{m \mid n+1}$ and $\# f=\# f^{(n)}$, and let $\theta \in \mathbb{N} \# f$ be fixed. If $L_{\theta}(f)=g$, then $g \in \mathbb{Z}_{++}^{m \mid n+1}$ and $g=\left(L_{\theta}\left(f^{(n)}\right), n+1\right)$. Similar statements hold for the operator $L^{\prime}$ as well.

Lemma 2.13. Let $n \in \mathbb{N}$ and $f=\left(f^{(n)}, n+1\right) \in \mathbb{Z}_{++}^{m \mid n+1}$ with $\# f=\# f^{(n)}$.

(1) Let $g \in \mathbb{Z}_{+}^{m \mid n+1}$ and $\theta \in \mathbb{N}^{\# g}$ such that $R_{\theta}(g)=f$. Then $g=\left(g^{(n)}, n+1\right) \in$ $\mathbb{Z}_{++}^{m \mid n+1}$ with $\# g=\# g^{(n)}$ and $R_{\theta}\left(g^{(n)}\right)=f^{(n)}$. 
(2) Let $\tilde{g} \in \mathbb{Z}_{+}^{m \mid n}$ and $\theta \in \mathbb{N}^{\# \tilde{g}}$ such that $R_{\theta}(\tilde{g})=f^{(n)}$. Then $\#(\tilde{g}, n+1)=\# \tilde{g}$ and $R_{\theta}((\tilde{g}, n+1))=f$.

Similar statements hold for the operator $R^{\prime}$ as well.

Lemmas 2.12, 2.13 and Corollary 2.10 imply the following $n=\infty$ analogue of [Br1, Theorem 3.34, Corollary 3.36].

Theorem 2.14. For $f \in \mathbb{Z}_{+}^{m \mid \infty}$ we have

(1) $U_{f}=\sum_{\theta \in\{0,1\} \# f} q^{|\theta|} K_{L_{\theta}(f)}$;

(2) $K_{f}=\sum_{\theta \in \mathbb{N} \# f}(-q)^{|\theta|} U_{L_{\theta}^{\prime}(f)}$;

(3) $K_{f}=\sum_{g} q^{-\left|\theta_{g}\right|} L_{g}$, where the sum is over all $g \in \mathbb{Z}_{+}^{m \mid \infty}$ such that $R_{\theta_{g}}(g)=f$ for some (unique) $\theta_{g} \in\{0,1\}^{\# g}$;

(4) $L_{f}=\sum(-q)^{-|\theta|} K_{g}$, summed over $g \in \mathbb{Z}_{+}^{m \mid \infty}$ and $\theta \in \mathbb{N}^{\# g}$ with $R_{\theta}^{\prime}(g)=f$.

Remark 2.15. Let $n \in \mathbb{N}$ and let $\mathbf{1}^{m \mid n}=(1, \cdots, 1 \mid 1, \cdots, 1)$. For $f, g \in \mathbb{Z}_{+}^{m \mid n}$, one has $\left(f-p \mathbf{1}^{m \mid n}\right)(n) \leq n,\left(g-p \mathbf{1}^{m \mid n}\right)(n) \leq n$ for $p$ large enough. One sees that

$$
u_{g, f}(q)=u_{g-p \mathbf{1}^{m \mid n}, f-p \mathbf{1}^{m \mid n}}(q), \quad \ell_{g, f}(q)=\ell_{g-p \mathbf{1}^{m \mid n}, f-p \mathbf{1}^{m \mid n}}(q) .
$$

The right-hand sides can be computed first by computing in $\widehat{\mathcal{E}}^{m \mid \infty}$ (Theorem 2.14) and then applying the truncation map $\mathfrak{T r}_{n}$. Thus, the structure of the (dual) canonical bases in $\widehat{\mathcal{E}}^{m \mid \infty}$ completely controls those in $\widehat{\mathcal{E}}^{m \mid n}$ via the truncation map.

\section{Representation theory of $\mathfrak{g l}(m \mid n)$}

The main goal of this section is to describe the relations between tilting modules, Kac modules, and irreducibles of $\mathfrak{g l}(m \mid \infty)$. To do this, we first study the connections between representations of $\mathfrak{g l}(m \mid n)$ and $\mathfrak{g l}(m \mid n+1)$.

3.1. The categories $\mathcal{O}_{m \mid n}^{+}$and $\mathcal{O}_{m \mid n}^{++}$. For $n \in \mathbb{N}$ the Lie superalgebra $\mathfrak{g}=\mathfrak{g l}(m \mid n)$ is generated by $e_{i j}$, where $i, j \in I(m \mid n)$. For $i \in I(m \mid n)$, let $\bar{i}=\overline{0}$ if $i>0$ and $i=\overline{1}$ if $i<0$. The subalgebra $\mathfrak{g}_{\overline{0}}$ of $\mathfrak{g}$ is generated by those $e_{i j}$ such that $\bar{i}+\bar{j}=\overline{0}$ and it is isomorphic to $\mathfrak{g l}(m) \oplus \mathfrak{g l}(n)$. Let $\mathfrak{h}$ be the standard Cartan subalgebra of $\mathfrak{g}$ consisting of all diagonal matrices, $\mathfrak{b}$ be the standard Borel subalgebra of all upper triangular matrices, and let $\mathfrak{p}=\mathfrak{g}_{0}+\mathfrak{b}$. The Lie superalgebra $\mathfrak{g}$ is endowed with a natural $\mathbb{Z}$-gradation

$$
\mathfrak{g}=\mathfrak{g l}(m \mid n)_{-1} \oplus \mathfrak{g l}(m \mid n)_{0} \oplus \mathfrak{g l}(m \mid n)_{+1},
$$

consistent with its $\mathbb{Z}_{2}$-gradation. Here $\mathfrak{g l}(m \mid n)_{ \pm 1}$ is the subalgebra spanned by the odd positive/negative root vectors. We let $\mathfrak{g l}(m \mid n)_{<0}=\mathfrak{g l}(m \mid n)_{0} \oplus \mathfrak{g l}(m \mid n)_{-1}$. By means of the natural inclusion $\mathfrak{g l}(m \mid n) \subseteq \mathfrak{g l}(m \mid n+1)$ via $I(m \mid n) \subseteq I(m \mid n+1)$, we let $\mathfrak{g l}(m \mid \infty):=\lim \mathfrak{g l}(m \mid n)$.

Let $\left.\left\{\delta_{i} \mid i \in I \overrightarrow{m \mid}\right)\right\}$ be the basis of $\mathfrak{h}^{*}$ dual to $\left\{e_{i i} \mid i \in I(m \mid n)\right\}$. Let $X_{m \mid n}$ be the set of integral weights $\lambda=\sum_{i \in I(m \mid n)} \lambda_{i} \delta_{i}, \lambda_{i} \in \mathbb{Z}$. A symmetric bilinear form on $\mathfrak{h}^{*}$ is defined by

$$
\left(\delta_{i} \mid \delta_{j}\right)=-\operatorname{sgn}(i) \delta_{i j}, \quad i, j \in I(m \mid n) .
$$

(Our bilinear form differs from the one in [Br1] by a sign.) Let $X_{m \mid n}^{+}$be the set of all $\lambda \in X_{m \mid n}$ such that $\lambda_{-m} \geq \cdots \geq \lambda_{-1}, \lambda_{1} \geq \cdots \geq \lambda_{n}$. Such a weight is called dominant. Let $X_{m \mid n}^{++}$be the set of all $\lambda \in X_{m \mid n}^{+}$with $\lambda_{n} \geq 0$. We may 
regard an element $\lambda$ in $X_{m \mid n}^{++}$as an element in $X_{m \mid n+1}^{++}$by letting $\lambda_{n+1}=0$ and let $X_{m \mid \infty}^{+}:=\lim _{m \mid n} X_{m \mid+}^{+}$. For $n \in \mathbb{N} \cup \infty$ define

$$
\rho=-\sum_{i \in I(m \mid n)} i \delta_{i} .
$$

Define a bijection

$$
X_{m \mid n} \longrightarrow \mathbb{Z}^{m \mid n}, \quad \lambda \mapsto f_{\lambda},
$$

where $f_{\lambda} \in \mathbb{Z}^{m \mid n}$ is given by $f_{\lambda}(i)=\left(\lambda+\rho \mid \delta_{i}\right)$, for $i \in I(m \mid n)$. This map induces bijections $X_{m \mid n}^{+} \rightarrow \mathbb{Z}_{+}^{m \mid n}$ (for $n$ possibly infinite), and $X_{m \mid n}^{++} \rightarrow \mathbb{Z}_{++}^{m \mid n}$ (for $n$ finite). Using this bijection we define the notions such as the degree of atypicality, the $\mathrm{L}$ and

$\mathrm{R}$ operators, $\epsilon$-weight, partial order $\preccurlyeq$, et cetera, for elements in $X_{m \mid n}^{+}$by requiring them to be compatible with those defined for elements in $\mathbb{Z}_{+}^{m \mid n}$.

For $\lambda \in X_{m \mid n}$, we define the Verma module and the Kac module to be

$$
M_{n}(\lambda):=U(\mathfrak{g}) \otimes_{U(\mathfrak{b})} \mathbb{C}_{\lambda} \text { and } K_{n}(\lambda):=U(\mathfrak{g}) \otimes_{U(\mathfrak{p})} L_{n}^{0}(\lambda),
$$

respectively. The irreducible module is denoted by $L_{n}(\lambda)$. Here $\mathbb{C}_{\lambda}$ is the standard one-dimensional module over $\mathfrak{h}$ extended trivially to $\mathfrak{b}$, and $L_{n}^{0}(\lambda)$ is the irreducible module of $\mathfrak{g}_{\overline{0}}$ of highest weight $\lambda$. Let $\left[M: L_{n}(\lambda)\right]$ denote the multiplicity of the irreducible module $L_{n}(\lambda)$ in a $\mathfrak{g l}(m \mid n)$-module $M$. When $n=\infty$ we will make it a convention to drop the subscript $n$.

For $n \in \mathbb{N}, \mathcal{O}_{m \mid n}^{+}$is the category of finite-dimensional $\mathfrak{g l}(m \mid n)$-modules $M$ with

$$
M=\bigoplus_{\gamma \in X_{m \mid n}} M_{\gamma},
$$

where as usual $M_{\gamma}$ denotes the $\gamma$-weight space of $M$ with respect to $\mathfrak{h}$. We denote by $\mathcal{O}_{m \mid n}^{++}$the full subcategory of $\mathcal{O}_{m \mid n}^{+}$which consists of modules whose composition factors are of the form $L_{n}(\lambda), \lambda \in X_{m \mid n}^{++}$.

We let $\mathcal{O}_{m \mid \infty}^{++}$be the category of $\mathfrak{h}$-semisimple finitely generated $\mathfrak{g l}(m \mid \infty)$-modules that are locally finite over $\mathfrak{g l}(m \mid N)$, for all finite $N$, and such that the composition factors are of the form $L(\lambda), \lambda \in X_{m \mid \infty}^{+}$.

Remark 3.1. When $n$ is finite one can pass all the relevant information between $\mathcal{O}_{m \mid n}^{+}$and $\mathcal{O}_{m \mid n}^{++}$by tensoring with the one-dimensional module $L_{n}\left(p \delta^{m \mid n}\right)$ for a suitable $p \in \mathbb{Z}$, where $\delta^{m \mid n}:=\sum_{i=-m}^{-1} \delta_{i}-\sum_{i=1}^{n} \delta_{i}$.

3.2. The truncation functor. Let $\operatorname{wt}(v)$ denote the weight (or $\delta$-weight) of a weight vector $v$ in a $\mathfrak{g l}(m \mid n)$-module.

Lemma 3.2. Let $n$ be finite.

(1) If $\lambda \in X_{m \mid n}^{++}$and $\left(\lambda \mid \delta_{n}\right)<0$ (respectively $\left.\leq 0\right)$, then for every weight vector $v$ in $K_{n}(\lambda)$ we have $\left(w t(v) \mid \delta_{n}\right)<0$ (respectively $\left.\leq 0\right)$.

(2) If $\lambda \in X_{m \mid n}^{++}$and $\left(\lambda \mid \delta_{n}\right)<0$ (respectively $\left.\leq 0\right)$, then for every weight vector $v$ in $L_{n}(\lambda)$ we have $\left(w t(v) \mid \delta_{n}\right)<0$ (respectively $\left.\leq 0\right)$.

(3) For every weight vector $v$ in any module $M \in \mathcal{O}_{m \mid n}^{++}$we have $\left(w t(v) \mid \delta_{n}\right) \leq 0$.

Proof. Since $K_{n}(\lambda)$ and $L_{n}(\lambda)$ are highest weight modules and every negative root $\alpha$ of $\mathfrak{g}$ satisfies $\left(\alpha \mid \delta_{n}\right) \leq 0,(1)$ and (2) are clear. Part (3) follows from (2). 
Corollary 3.3. For $n \in \mathbb{N}$ and $\lambda \in X_{m \mid n}^{++}$we have $K_{n}(\lambda) \in \mathcal{O}_{m \mid n}^{++}$.

Definition 3.4. For $n<n^{\prime} \leq \infty$, the truncation functor $\mathfrak{t r}_{n^{\prime}, n}: \mathcal{O}_{m \mid n^{\prime}}^{++} \longrightarrow \mathcal{O}_{m \mid n}^{++}$is defined by sending an object $M$ to

$$
\mathfrak{t r}_{n^{\prime}, n}(M):=\operatorname{span}\left\{v \in M \mid\left(\operatorname{wt}(v) \mid \delta_{k}\right)=0, \text { for all } n+1 \leq k \leq n^{\prime}\right\} .
$$

When $n^{\prime}$ is clear from the context we will also write $\mathfrak{t r}_{n}$ for $\mathfrak{t r}_{n^{\prime}, n}$.

We have a system of categories $\mathcal{O}_{m \mid n}^{++}$with a compatible sequence of functors $\mathfrak{t r}_{k, n}$ in the sense that $\mathfrak{t r}_{n^{\prime \prime}, n}=\mathfrak{t r}_{n^{\prime}, n} \circ \mathfrak{t r}_{n^{\prime \prime}, n^{\prime}}$ for $n^{\prime \prime}>n^{\prime}>n$.

\subsection{Kac and irreducible modules in $\mathcal{O}_{m \mid n}^{++}$and $\mathcal{O}_{m \mid \infty}^{++}$.}

Lemma 3.5. Let $n$ be finite. For $\lambda \in X_{m \mid n}^{++}$, we have the following natural inclusions of $\mathfrak{g l}(m \mid n)$-modules:

$$
L_{n}(\lambda) \subseteq L_{n+1}(\lambda), \quad K_{n}(\lambda) \subseteq K_{n+1}(\lambda) .
$$

Proof. The eigenvalue of the operator $e_{n+1, n+1} \in \mathfrak{g l}(m \mid n+1)$ provides an $\mathbb{N}$ gradation on the Kac module $K_{n+1}(\lambda)$, whose degree zero subspace is isomorphic to $K_{n}(\lambda)$. Similarly, we have $L_{n}(\lambda) \subseteq L_{n+1}(\lambda)$.

By Lemma 3.5 and the natural inclusions $\mathfrak{g l}(m \mid 1) \subset \cdots \subset \mathfrak{g l}(m \mid \infty), \bigcup_{n} K_{n}(\lambda)$ and $\bigcup_{n} L_{n}(\lambda)$ are naturally $\mathfrak{g l}(m \mid \infty)$-modules. They are direct limits of $\left\{K_{n}(\lambda)\right\}$ and $\left\{L_{n}(\lambda)\right\}$ and isomorphic to $K(\lambda)$ and $L(\lambda)$, respectively. Similarly $\bigcup_{n} L_{n}^{0}(\lambda)$ is an irreducible $\mathfrak{g l}(m) \oplus \mathfrak{g l}(\infty)$-module. The proof of Lemma 3.5 implies the following.

Corollary 3.6. Let $n<n^{\prime} \leq \infty$. Let $\lambda=\left(\lambda_{-m}, \cdots, \lambda_{n^{\prime}}\right) \in X_{m \mid n^{\prime}}^{++}$if $n^{\prime}$ is finite, and $\lambda \in X_{m \mid \infty}^{+}$otherwise. Then, for $Y=L$ or $K$ we have

$$
\mathfrak{t r}_{n^{\prime}, n}\left(Y_{n^{\prime}}(\lambda)\right)=\left\{\begin{array}{rr}
Y_{n}(\lambda), & \text { if } \lambda_{i}=0, \forall i>n, \\
0, & \text { otherwise. }
\end{array}\right.
$$

Remark 3.7. Fix $\lambda \in X_{m \mid n_{0}}^{++}$. Let $n \geq n_{0}$ and regard $\lambda \in X_{m \mid n}^{++}$. Let $\mathfrak{J}_{n}$ denote the set of the highest weights of the composition factors of $K_{n}(\lambda)$ and $r(n)$ denote the length of a composition series of $K_{n}(\lambda)$. By [Br1], $\mathfrak{J}_{n}$ consists precisely of those weights $\mu$ such that $\mathrm{R}_{\theta}(\mu)=\lambda$, for some $\theta \in\{0,1\}^{\# \lambda}$. As the operator $\mathrm{R}_{\theta}$ only affects the atypical parts of $\mu$, we see that $r(n)$ and $\mathfrak{J}_{n}$ (with the tail of zeros in a weight ignored) are independent of $n$, once we have chosen $n$ large enough so that $\# \lambda$ is the same when $\lambda$ is regarded as an element in $X_{m \mid n}^{++}$.

Lemma 3.8. Let $\lambda \in X_{m \mid \infty}^{+}$with $\lambda_{i}=0$ for $i \geq n_{0}$, and regard $\lambda \in X_{m \mid n}^{++}$for $n \geq n_{0}$. Suppose $n_{0}$ is chosen so that every $K_{n}(\lambda)$, for $n \geq n_{0}$, has the same number of composition factors (see Remark 3.7). Then $\mathfrak{t r}_{n^{\prime}, n}$ maps bijectively the set of Jordan-Hölder series of $K_{n^{\prime}}(\lambda)$ onto the set of Jordan-Hölder series of $K_{n}(\lambda)$, for all $n \leq n^{\prime} \leq \infty$. In particular $[K(\lambda): L(\mu)]=\left[K_{n}(\lambda): L_{n}(\mu)\right]$ for every $n \geq n_{0}$.

Proof. We denote by $U(\mathfrak{g l}(m \mid n))_{+}$, respectively $U(\mathfrak{g l}(m \mid n))_{-}$, the subalgebra of $U(\mathfrak{g l}(m \mid n))$ generated by the positive, respectively negative, root vectors of $\mathfrak{g l}(m \mid n)$.

For $n$ finite let $0 \subsetneq V_{n}^{1} \subsetneq V_{n}^{2} \subsetneq \cdots \subsetneq V_{n}^{r}=K_{n}(\lambda)$ be a composition series of $K_{n}(\lambda)$ with composition factors $V_{n}^{i} / V_{n}^{i-1} \cong L_{n}\left(\lambda^{i}\right)$. Attached to it we have a set of weight vectors, say $\left\{v^{1}, \cdots, v^{r}\right\}$, determined in such a way that the 
irreducible module $L_{n}\left(\lambda^{i}\right) \cong V_{n}^{i} / V_{n}^{i-1}$ is generated by the highest weight vector $v^{i}+V_{n}^{i-1}$ of highest weight $\lambda^{i}$. Viewing $v^{k} \in K_{n}(\lambda) \subseteq K_{n+1}(\lambda)$, we let $V_{n+1}^{i}:=\sum_{k=1}^{i} U(\mathfrak{g l}(m \mid n+1))_{-} v^{k}$. Now by Lemma 3.2. $e_{l, n+1} v^{k}=0$ for $l \leq n$, and hence $V_{n+1}^{i}$ is a $\mathfrak{g l}(m \mid n+1)$-submodule of $K_{n+1}(\lambda)$. Also $V_{n+1}^{i} / V_{n+1}^{i-1} \neq 0$, and so $0 \subsetneq V_{n+1}^{1} \subsetneq V_{n+1}^{2} \subsetneq \cdots \subsetneq V_{n+1}^{r}=K_{n+1}(\lambda)$ is a composition series for $K_{n+1}(\lambda)$. In this way we have defined a map $\phi_{n, n+1}$, which takes a composition series of $K_{n}(\lambda)$ to a composition series of $K_{n+1}(\lambda)$.

By construction $V_{n+1}^{i}=V_{n}^{i} \oplus C^{i}$, where $C^{i}$ is a $\mathfrak{g l}(m \mid n)$-submodule on which $e_{n+1, n+1}$ acts non-trivially, and thus $\mathfrak{t r}_{n+1, n} \circ \phi_{n, n+1}$ is the identity. Now for a composition series of $K_{n+1}(\lambda)$ let $\left\{v^{1}, \cdots, v^{r}\right\}$ be defined as before. It is clear that $\left\{v^{1}, \cdots, v^{r}\right\}$ also gives rise to a composition series for $\mathfrak{t r}_{n+1, n}\left(K_{n+1}(\lambda)\right)=K_{n}(\lambda)$. By construction of $\phi_{n, n+1}$ this composition series of $K_{n}(\lambda)$ lifts to the original composition series of $K_{n+1}(\lambda)$, and hence $\phi_{n, n+1} \circ \mathfrak{t r}_{n+1, n}$ is the identity.

Now let $V^{i}$ be the $\mathfrak{g l}(m \mid \infty)$-module $\bigcup_{n} V_{n}^{i}$. Since $v^{i} \notin V^{i-1}$, we have $0 \subsetneq V^{1} \subsetneq$ $V^{2} \subsetneq \cdots \subsetneq V^{r}=K(\lambda)$. It is straightforward to verify that each factor module $V^{i} / V^{i-1}$ is irreducible and hence isomorphic to $L\left(\lambda^{i}\right)$ by construction. Bijection of composition series now is established as before.

Corollary 3.9. The Kac module $K(\lambda)$ for $\lambda \in X_{m \mid \infty}^{+}$has a finite composition series. Furthermore its composition factors are of the form $L(\mu)$ with $\mu \in X_{m \mid \infty}^{+}$, and thus $K(\lambda)$ belongs to the category $\mathcal{O}_{m \mid \infty}^{++}$.

3.4. Relating tilting modules in $\mathcal{O}_{m \mid n}^{++}$and $\mathcal{O}_{m \mid n+1}^{++}$. Throughout this subsection we assume that $n$ is finite. An object $M \in \mathcal{O}_{m \mid n}^{+}$is said to have a Kac flag if it has a filtration of $\mathfrak{g l}(m \mid n)$-modules:

$$
0=M_{0} \subseteq \cdots \subseteq M_{r}=M
$$

such that each $M_{i} / M_{i-1}$ is isomorphic to $K_{n}\left(\lambda^{i}\right)$ for some $\lambda^{i} \in X_{m \mid n}^{+}$. We define $\left(M: K_{n}(\mu)\right)$ for $\mu \in X_{m \mid n}^{+}$to be the number of subquotients of a Kac flag of $M$ that are isomorphic to $K_{n}(\mu)$.

Definition 3.10. The tilting module associated to $\lambda \in X_{m \mid n}^{+}$in the category $\mathcal{O}_{m \mid n}^{+}$ is the unique indecomposable $\mathfrak{g l}(m \mid n)$-module $U_{n}(\lambda)$ satisfying:

(1) $U_{n}(\lambda)$ has a Kac flag with $K_{n}(\lambda)$ at the bottom,

(2) $\operatorname{Ext}^{1}\left(K_{n}(\mu), U_{n}(\lambda)\right)=0$, for all $\mu \in X_{m \mid n}^{+}$.

Let $n \in \mathbb{N}$ and $\lambda \in X_{m \mid n}^{++}$. By Corollary 3.3 and Theorem 4.37 (i) of [Br1] we see that $U_{n}(\lambda) \in \mathcal{O}_{m \mid n}^{++}$. Now let $\lambda=\left(\lambda_{-m}, \cdots, \lambda_{n+1}\right) \in X_{m \mid n+1}^{++}$and let $U_{n+1}(\lambda)$ be the tilting module of $\mathfrak{g l}(m \mid n+1)$ corresponding to $\lambda$. Let $\# \lambda$ denote the degree of atypicality of $\lambda$. By $\mathrm{Br1}, U_{n+1}(\lambda)$ has a Kac flag of the form:

$$
0=U_{n+1}^{0} \subsetneq U_{n+1}^{1} \subsetneq U_{n+1}^{2} \subsetneq \cdots \subsetneq U_{n+1}^{a_{n+1}}=U_{n+1}(\lambda),
$$

where $U_{n+1}^{i} / U_{n+1}^{i-1} \cong K_{n+1}\left(\lambda^{i, n+1}\right)$ for $i=1, \cdots, a_{n+1}$ and $\lambda^{1, n+1}=\lambda$. Furthermore $a_{n+1}=2^{\# \lambda}$, and the $\lambda^{i, n+1}$ 's are of the form $\lambda^{\mathrm{L}_{\theta}}$, where $\theta=\left(\theta_{1}, \cdots, \theta_{\# \lambda}\right) \in$ $\{0,1\}^{\# \lambda}$.

If $\left(\lambda \mid \delta_{n+1}\right)<0$, then $\left(\lambda^{\mathrm{L}_{\theta}} \mid \delta_{n+1}\right)<0$ for every $\theta$, since $\lambda^{\mathrm{L}_{\theta}} \preccurlyeq \lambda$. Thus by Lemma $3.2 \mathfrak{r}_{n}\left(U_{n+1}(\lambda)\right)=0$. 
Now assume that $\left(\lambda \mid \delta_{n+1}\right)=0$. Note that the degree of atypicality of $\lambda$, regarded as an element in $X_{m \mid n}^{++}$, is either $\# \lambda-1$ or $\# \lambda$, depending on whether $\lambda_{n+1}=0$ affects the atypicality. If it is $\# \lambda$, then $\lambda_{n+1}$ is not an atypical part of $\lambda$. Since the operators $\mathrm{L}_{\theta}$ only affect the atypical part of $\lambda$, it follows that $\left(\lambda^{\mathrm{L}_{\theta}} \mid \delta_{n+1}\right)=\left(\lambda \mid \delta_{n+1}\right)=0$ for each $\theta$, and the two sets $\left\{\lambda^{1, n}, \cdots, \lambda^{a_{n}, n}\right\}$ and $\left\{\lambda^{1, n+1}, \cdots, \lambda^{a_{n+1}, n+1}\right\}$ are identical.

On the other hand, if the degree of atypicality is $\# \lambda-1$, then for $\theta$ of the form $\left(1, \theta_{2}, \cdots, \theta_{\# \lambda}\right)$, we have $\left(\lambda^{\mathrm{L}_{\theta}} \mid \delta_{n+1}\right)<0$, and thus these $2^{\# \lambda-1}$ Kac modules will not contribute to $\mathfrak{t r}_{n}\left(U_{n+1}(\lambda)\right)$. The remaining $2^{\# \lambda-1}$ Kac modules have highest weights satisfying $\left(\lambda^{\mathrm{L}_{\theta}} \mid \delta_{n+1}\right)=0$, and under $\mathfrak{t r}_{n}$ will contribute $K_{n}\left(\lambda^{\mathrm{L}_{\theta}}\right)$ in a Kac flag of $\mathfrak{t r}_{n}\left(U_{n+1}(\lambda)\right)$. But these are precisely the factors in a Kac flag of $U_{n}(\lambda)$.

Denote by $J_{n}(\lambda)=\left\{\lambda^{1, n}, \cdots, \lambda^{a_{n}, n}\right\}$ the set of the highest weights of the Kac modules of a Kac flag of $U_{n}(\lambda)$. Summarizing we have the following.

Proposition 3.11. Let $\lambda \in X_{m \mid n+1}^{++}$with $\left(\lambda \mid \delta_{n+1}\right)=0$, and $\# \lambda$ be its degree of atypicality. Then $J_{n}(\lambda)$ consists of those $\mu$ 's from $J_{n+1}(\lambda)$ with $\left(\mu \mid \delta_{n+1}\right)=0$.

Proposition 3.12. Let $\lambda \in X_{m \mid \infty}^{+}$be such that $\lambda_{i}=0$ for $i \geq n_{0}$, and regard $\lambda \in X_{m \mid n}^{++}$for $n \geq n_{0}$. Suppose that $n_{0}$ is chosen such that the degree of atypicality of $\lambda$ regarded as an element in $X_{m \mid n}^{+}$is the same for $n \geq n_{0}$. Then $J_{n}(\lambda)=J_{n_{0}}(\lambda)$.

Proposition 3.13. For $\lambda \in X_{m \mid n+1}^{++}$the map $\operatorname{tr}_{n}$ sends $U_{n+1}(\lambda)$ to $U_{n}(\lambda)$, if $\left(\lambda \mid \delta_{n+1}\right)=0$, and to 0 , otherwise.

Proof. By Br1], $U_{n+1}(\lambda)$ has a Kac flag with subquotients isomorphic to $K_{n+1}(\mu)$, where $\lambda^{\mathrm{L}} \preccurlyeq \mu \preccurlyeq \lambda$. If $\left(\lambda \mid \delta_{n+1}\right)>0$, then Corollary 3.6 implies $\mathfrak{t r}_{n}\left(U_{n+1}(\lambda)\right)=0$.

Let $\lambda \in X_{m \mid n+1}^{++}$with $\left(\lambda \mid \delta_{n+1}\right)=0$ and consider $U_{n+1}(\lambda)$. Let

$$
\partial:=\frac{1}{2}\left(\sum_{i<0} e_{i i}-\sum_{j>0} e_{j j}\right) .
$$

Then $\partial$ equips $\mathfrak{g l}(m \mid n+1)$ with the $\mathbb{Z}$-gradation (3.1), which allows us to regard $\mathcal{O}_{m \mid n+1}^{++}$as a $\mathbb{Z}$-graded module category with gradation-preserving morphisms as in $\mathrm{So3}, \mathrm{Br} 2$. By the construction of So3] one has a filtration of indecomposable modules $3=\lambda(\partial):=|\lambda|)$

$$
K_{n+1}(\lambda)=T_{\geq a} \subseteq T_{\geq a-1} \subseteq T_{\geq a-2} \subseteq \cdots \subseteq T_{\geq r}=U_{n+1}(\lambda)
$$

According to Br1 tilting modules have multiplicity-free Kac flags, we have

$$
T_{\geq i-1} / T_{\geq i} \cong \bigoplus_{|\mu|=i-1} K_{n+1}(\mu),
$$

where the summation is over those $\mu$ 's in $X_{m \mid n+1}^{++}$with $\operatorname{Ext}^{1}\left(K_{n+1}(\mu), T_{\geq i}\right) \neq 0$. Furthermore $T_{\geq i-1}$ contains a non-trivial extension of $K_{n+1}(\mu)$ by $T_{\geq i}$ for every such $\mu$. Applying the truncation functor $\mathfrak{t r}_{n}$ to (3.4) we obtain a filtration

$$
K_{n}(\lambda) \subseteq S_{\geq a-1} \subseteq S_{\geq a-2} \subseteq \cdots \subseteq S_{\geq r}
$$

with $S_{\geq i-1} / S_{\geq i} \cong \bigoplus_{|\mu|=i-1} K_{n}(\mu)$, and with summation over $\mu$ with $\left(\mu \mid \delta_{n+1}\right)=0$.

\footnotetext{
${ }^{3} \mathrm{It}$ is asserted in $\mathrm{So} 3$ that the modules $T_{>k}$ are indecomposable. A simple proof of this fact can be read off from the proof of Proposition 3.1 in [So4. We learned of this proof from W. Soergel through the anonymous expert, and we thank both of them.
} 
We claim that the extension of each $K_{n}(\mu)$ by $S_{\geq i}$ in 3.5) is non-trivial, for $\mu$ satisfying $\left(\mu \mid \delta_{n+1}\right)=0,|\mu|=i-1$, and $\operatorname{Ext}^{1}\left(K_{n+1}(\mu), T_{\geq i}\right) \neq 0$. Suppose it were trivial for some $\mu$. Let us denote the extension of $K_{n+1}(\mu)$ by $T_{\geq i}$ by $T_{>i}^{\mu}$ and set $S_{\geq i}^{\mu}=\mathfrak{t r}_{n}\left(T_{\geq i}^{\mu}\right)$. Let $w \in T_{\geq i}^{\mu}$ of weight $\mu$ be such that its image in $T_{\geq i}^{\bar{\mu}} / T_{\geq i}$ generates over $\mathfrak{g l}(m \mid n+1)_{\leq 0}$ a Kac module $K_{n+1}(\mu)$. Since $S_{\geq_{i}}^{\mu}$ is a trivial extension of $K_{n}(\mu)$ by $S_{\geq i}$, we may assume (by adding to $w$ an element from $S_{\geq i}$ if necessary) that $e_{i j} w=0$, for all $-m \leq i<j \leq n$. By Lemma 3.2. $e_{l, n+1} w=0$, for $l \leq n$, and hence $w$ is a genuine $\mathfrak{g l}(m \mid n+1)$-highest weight vector of highest weight $\mu$ in $T_{\geq i-1}$. This implies that the extension $T_{\geq i}^{\mu}$ is split, which is a contradiction.

Now Proposition 3.11 and Soergel's construction for $U_{n}(\lambda)$ imply that (3.5) is a construction of the tilting module $U_{n}(\lambda)$ and hence $S_{\geq r} \cong U_{n}(\lambda)$.

Corollary 3.14. Let $\lambda \in X_{m \mid \infty}^{+}$with $\lambda_{i}=0$ for $i \geq n_{0}$, and regard $\lambda \in X_{m \mid n}^{++}$ for $n \geq n_{0}$. Suppose that $n_{0}$ is such that $J_{n}(\lambda)=J_{n_{0}}(\lambda)$, for $n \geq n_{0}$. Then $U_{n_{0}}(\lambda) \subseteq U_{n}(\lambda)$ and $\left(U_{n}(\lambda): K_{n}(\mu)\right)=\left(U_{n_{0}}(\lambda): K_{n_{0}}(\mu)\right)$. Furthermore a Kac flag of $U_{n}(\lambda)$, for every $n \geq n_{0}$, can be chosen to have the same ordered sequence of weights.

Proof. Consider the construction of $U_{n_{0}}(\lambda)$ as in (3.4). We define a total order on the $\mu$ 's that appear in the construction by requiring that $\mu>\nu$ if $|\mu|>|\nu|$, and among the $\mu$ 's with the same $|\mu|$ we choose an arbitrary total order. Starting with $K_{n_{0}}(\lambda)$, following this total order, we construct $U_{n_{0}}(\lambda)$. This gives a Kac flag of $U_{n_{0}}(\lambda)$. Regarding the same $\mu$ 's as weights of $\mathfrak{g l}\left(m \mid n_{0}+1\right)$ we construct in the same fashion $U_{n_{0}+1}(\lambda)$ with the a Kac flag having the same sequence of weights.

3.5. Tilting modules in $\mathcal{O}_{m \mid \infty}^{++}$. In the same way as in Definition 3.10 one defines tilting modules $U(\lambda)$ in the category $\mathcal{O}_{m \mid \infty}^{++}$, where $\lambda \in X_{m \mid \infty}^{+}$. The following lemma is standard using induction and the long exact sequence.

Lemma 3.15. Let $U$ be a $\mathfrak{g r}(m \mid \infty)$-module such that $\operatorname{Ext}^{1}(K(\mu), U)=0$, for all $\mu$. If $V$ is a $\mathfrak{g l}(m \mid \infty)$-module possessing a finite Kac flag, then we have $\operatorname{Ext}^{1}(V, U)=0$.

Theorem 3.16. Let $\lambda \in X_{m \mid \infty}^{+}$.

(1) There exists a unique (up to isomorphism) tilting module associated to $\lambda$ in $\mathcal{O}_{m \mid \infty}^{++} \cdot$ Moreover, $U(\lambda) \cong \bigcup_{n} U_{n}(\lambda)$.

(2) The functor $\mathfrak{t r}_{n}$ sends $U(\lambda)$ to $U_{n}(\lambda)$ if $\left(\lambda \mid \delta_{n+1}\right)=0$ and to 0 otherwise.

(3) We have $(U(\lambda): K(\mu))=\left(U_{n}(\lambda): K_{n}(\mu)\right)$ for $n \gg 0$, and hence $U(\lambda)$ has a Kac flag consisting of Kac modules of highest weights $\mu$ with $\lambda^{L} \preccurlyeq \mu \preccurlyeq \lambda$.

Proof. Corollary 3.14 allows us to define the $\mathfrak{g l}(m \mid \infty)$-module $U(\lambda)$ to be

$$
U(\lambda)=\bigcup_{n} U_{n}(\lambda) .
$$

Parts (2) and (3) follow from Corollary 3.14.

It remains to prove (1). First by Corollary 3.14 $U(\lambda)$ admits a Kac flag with the same ordered sequence of weights as $U_{n}(\lambda)$, for $n \gg 0$. Now for $\lambda \in X_{m \mid n}^{++}$the highest weights of the Kac modules that appear in a Kac flag of $U_{n}(\lambda)$ lie in $X_{m \mid n}^{++}$. Also the highest weights of the composition factors of a Kac module with highest weight lying in $X_{m \mid n}^{++}$also lie in $X_{m \mid n}^{++}$. Thus $U(\lambda)$ has a finite composition series 
and all its composition factors have highest weights lying in $X_{m \mid \infty}^{+}$. Thus $U(\lambda)$ belongs to the category $\mathcal{O}_{m \mid \infty}^{++}$.

Suppose $U(\lambda)=M_{1} \oplus M_{2}$ as $\mathfrak{g l}(m \mid \infty)$-modules. Choose $n$ so that $\left(\lambda_{k} \mid \delta_{n}\right)=0$, for all $\lambda_{k}$, where the $\lambda_{k}$ 's are all the highest weights of composition factors of $U(\lambda)$ (and hence of $M_{1}$ and $M_{2}$ as well). Now $U_{n}(\lambda)=\mathfrak{t r}_{n}\left(M_{1}\right) \oplus \mathfrak{t r}_{n}\left(M_{2}\right)$. But $M_{j} \neq 0$ would imply $\operatorname{tr}_{n}\left(M_{j}\right) \neq 0$, which contradicts the indecomposability of $U_{n}(\lambda)$. Thus, $U(\lambda)$ is indecomposable.

Given an exact sequence of $\mathfrak{g l}(m \mid \infty)$-modules of the form

$$
0 \longrightarrow U(\lambda) \longrightarrow M \longrightarrow K(\mu) \longrightarrow 0
$$

where $\mu \in X_{m \mid \infty}^{+}$, we apply $\mathfrak{t r}_{n}$ to (3.6) and obtain a split exact sequence

$$
0 \longrightarrow U_{n}(\lambda) \longrightarrow \mathfrak{t r}_{n}(M) \longrightarrow K_{n}(\mu) \longrightarrow 0
$$

of $\mathfrak{g l}(m \mid n)$-modules. Thus, we can choose $v_{\mu} \in \mathfrak{t r}_{n}(M)$ of weight $\mu$ such that $e_{i j} v_{\mu}=0$, for all $-m \leq i<0<j \leq n$. Observe that $e_{k l} v_{\mu}=0$ for $k, l$ such that $k<l$ and $l>n$ by Lemma 3.2. Thus $v_{\mu}$ is a genuine $\mathfrak{g l}(m \mid \infty)$-highest weight vector and hence (3.6) is split. Thus $U(\lambda)$ is a tilting module.

A standard Fitting-type argument as in So3 proves the uniqueness of $U(\lambda)$.

Denote by $G\left(\mathcal{O}_{m \mid \infty}^{++}\right)$the Grothendieck group of $\mathcal{O}_{m \mid \infty}^{++}$and let $G\left(\mathcal{O}_{m \mid \infty}^{++}\right)_{\mathbb{Q}}:=$ $G\left(\mathcal{O}_{m \mid \infty}^{++}\right) \otimes_{\mathbb{Z}} \mathbb{Q}$. For $M \in \mathcal{O}_{m \mid \infty}^{++}$let $[M]$ denote the corresponding element in $G\left(\mathcal{O}_{m \mid \infty}^{++}\right) \mathbb{Q}$. Let $\mathcal{E}_{q=1}^{m \mid \infty}$ denote the specialization of $\mathcal{E}^{m \mid \infty}$ at $q \rightarrow 1$. The topological completion $\widehat{\mathcal{E}}^{m \mid \infty}$ of $\mathcal{E}^{m \mid \infty}$ induces a topological completion $\widehat{\varepsilon}_{q=1}^{m \mid \infty}$ of $\varepsilon_{q=1}^{m \mid \infty}$. Using the bijection between $\mathcal{E}_{q=1}^{m \mid \infty}$ and $G\left(\mathcal{O}_{m \mid \infty}^{++}\right) \mathbb{Q}$, induced by sending $K_{f_{\lambda}}$ to $[K(\lambda)]$ for $\lambda \in X_{m \mid \infty}^{+}$, we may define a topological completion $\widehat{G}\left(\mathcal{O}_{m \mid \infty}^{++}\right)_{\mathbb{Q}}$ of $G\left(\mathcal{O}_{m \mid \infty}^{++}\right) \mathbb{Q}$.

Proposition 3.17. (1) The linear map $j:\left.\widehat{G}\left(\mathcal{O}_{m \mid \infty}^{++}\right)_{\mathbb{Q}} \rightarrow \widehat{\mathcal{E}}^{m \mid \infty}\right|_{q=1}$ that sends $[K(\lambda)]$ to $K_{f_{\lambda}}(1)$ for each $\lambda \in X_{m \mid \infty}^{+}$is an isomorphism of vector spaces.

(2) $j$ sends $[U(\lambda)]$ to $U_{f_{\lambda}}(1)$ for each $\lambda \in X_{m \mid \infty}^{+}$.

(3) $j$ sends $[L(\lambda)]$ to $L_{f_{\lambda}}(1)$ for each $\lambda \in X_{m \mid \infty}^{+}$.

Proof. Clearly $j$ is an isomorphism of vector spaces. Write

$$
[K(\lambda)]=\sum_{\mu} a_{\lambda, \mu}[L(\mu)], \quad\left[K_{n}(\lambda)\right]=\sum_{\mu} a_{\lambda, \mu ; n}\left[L_{n}(\mu)\right],
$$

for some $a_{\lambda, \mu}, a_{\lambda, \mu ; n} \in \mathbb{Z}$. Lemma 3.8 says that $a_{\lambda, \mu}=a_{\lambda, \mu ; n}$ for $n \gg 0$ relative to $\lambda, \mu$. Corollary 2.10 implies that $b_{\lambda, \mu}=b_{\lambda, \mu ; n}$ for $n \gg 0$ if we write

$$
K_{f_{\lambda}}=\sum_{\mu} b_{\lambda, \mu} L_{f_{\mu}}, \quad K_{f_{\lambda}^{(n)}}=\sum_{\mu} b_{\lambda, \mu ; n} L_{f_{\mu}^{(n)}} .
$$

The finite $n$ analogue of (3) in Br1] says that $a_{\lambda, \mu ; n}=b_{\lambda, \mu ; n}$. Thus (3) follows.

In a similar fashion (2) follows from Corollary 2.10, Theorem 3.16 , and Brundan's finite $n$ analogue of (2).

Remark 3.18. We note that the operator L : $X_{m \mid \infty}^{+} \rightarrow X_{m \mid \infty}^{+}$is not surjective in contrast to the finite $n$ case. For example the trivial weight does not lie in the image of L. 


\section{Kazhdan-Lusztig polynomials And CANONiCAL BASES FOR $\mathcal{E}^{m+n}$}

The goal of this section is to establish closed formulas for the (dual) canonical bases and Kazhdan-Lusztig polynomials in the Fock space $\varepsilon^{m+n}$. We begin with some standard results on canonical bases on tensor and exterior spaces, which seem to be well known (cf. e.g. [Lu2, FKK, Br1]).

4.1. Bases for $\mathbb{T}^{m+n}$. We say that $f \in \mathbb{Z}^{m+n}$ is anti-dominant if $f(-m) \leq \cdots \leq$ $f(-1) \leq f(1) \leq \cdots \leq f(n)$. The following is standard.

Proposition 4.1. Let $n$ be finite. There exists a unique anti-linear bar map ${ }^{-}$: $\mathbb{T}^{m+n} \rightarrow \mathbb{T}^{m+n}$ such that

(1) $\overline{\nu_{f}}=V_{f}$ for all anti-dominant $f \in \mathbb{Z}^{m+n}$;

(2) $\overline{X u H}=\bar{X} \bar{u} \bar{H}$ for all $X \in \mathcal{U}, u \in \mathbb{T}^{m+n}, H \in \mathcal{H}_{m+n}$;

(3) the bar map is an involution;

(4) $\overline{\mathcal{V}_{f}}=\mathcal{V}_{f}+(*)$ where $(*)$ is a finite $\mathbb{Z}\left[q, q^{-1}\right]$-linear combination of $\mathcal{V}_{g}$ 's for $g<f$.

By standard arguments again, Proposition 4.1 implies the existence and uniqueness of the canonical basis $\left\{\mathcal{T}_{f}\right\}$ and dual canonical basis $\left\{\mathcal{L}_{f}\right\}$ for $\mathbb{T}^{m+n}$.

Theorem 4.2. Let $n$ be finite. There exist unique bases $\left\{\mathcal{T}_{f}\right\},\left\{\mathcal{L}_{f}\right\}$ for $\mathbb{T}^{m+n}$, where $f \in \mathbb{Z}^{m+n}$, such that

(1) $\overline{\mathcal{T}}_{f}=\mathcal{T}_{f}$ and $\overline{\mathcal{L}}_{f}=\mathcal{L}_{f}$;

(2) $\mathcal{T}_{f} \in \mathcal{V}_{f}+\sum_{g \in \mathbb{Z}^{m+n}} q \mathbb{Z}[q] \mathcal{V}_{g}$ and $\mathcal{L}_{f} \in \mathcal{V}_{f}+\sum_{g \in \mathbb{Z}^{m+n}} q^{-1} \mathbb{Z}\left[q^{-1}\right] \mathcal{V}_{g}$;

(3) $\mathcal{T}_{f}=\mathcal{V}_{f}+(*)$ and $\mathcal{L}_{f}=\mathcal{V}_{f}+(* *)$ where $(*)$ and $(* *)$ are finite $\mathbb{Z}\left[q, q^{-1}\right]$ linear combinations of $\mathcal{V}_{g}$ 's for $g \in \mathbb{Z}^{m+n}$ with $g<f$.

We define $\mathfrak{t}_{g, f}(q) \in \mathbb{Z}[q], \mathfrak{l}_{g, f}(q) \in \mathbb{Z}\left[q^{-1}\right]$ associated to $f, g \in \mathbb{Z}^{m+n}$ by

$$
\mathcal{T}_{f}=\sum_{g \in \mathbb{Z}^{m+n}} \mathfrak{t}_{g, f}(q) \mathcal{V}_{g}, \quad \mathcal{L}_{f}=\sum_{g \in \mathbb{Z}^{m+n}} \mathfrak{l}_{g, f}(q) \mathcal{V}_{g} .
$$

Note that $\mathfrak{t}_{g, f}(q)=\mathfrak{l}_{g, f}(q)=0$ unless $g \leq f$ and $\mathfrak{t}_{f, f}(q)=\mathfrak{l}_{f, f}(q)=1$. We will refer to these polynomials as Kazhdan-Lusztig polynomials (see Proposition 5.3).

Remark 4.3. One can show that the matrices $\left[\mathfrak{t}_{-f,-g}\left(q^{-1}\right)\right]$ and $\left[\mathfrak{l}_{f, g}(q)\right]$ are inverses of each other. In light of Proposition 5.3, this can be regarded as a reformulation of the inversion formula of the parabolic Kazhdan-Lusztig polynomials. Alternatively, this follows from similar arguments as in $\S 2$-i of Br1]. Thus (4.1) implies the following duality formulas:

$$
\mathcal{V}_{f}=\sum_{g \in \mathbb{Z}^{m+n}} \mathfrak{t}_{-f,-g}\left(q^{-1}\right) \mathcal{L}_{g}=\sum_{g \in \mathbb{Z}^{m+n}} \mathfrak{l}_{-f,-g}\left(q^{-1}\right) \mathcal{T}_{g}, \quad f \in \mathbb{Z}^{m+n}
$$

4.2. Bases for $\mathcal{E}^{m+n}$ and $\widehat{\mathcal{\varepsilon}}^{m+\infty}$. For $n \in \mathbb{N}$, let $\mathcal{E}_{+}^{m+n}$ denote the subspace of $\mathcal{E}^{m+n}$ spanned by elements of the form $\mathcal{K}_{f}, f \in \mathbb{Z}_{++}^{m+n}$. Define the truncation map $\operatorname{Tr}_{n+1, n}: \mathcal{E}_{+}^{m+(n+1)} \longrightarrow \mathcal{E}_{+}^{m+n}$ by sending $\mathcal{K}_{f}$ to $\mathcal{K}_{f(n)}$ if $f(n+1)=-n$, and to 0 otherwise. For $n^{\prime}>n$, $\operatorname{Tr}_{n^{\prime}, n}: \varepsilon_{+}^{m+n^{\prime}} \longrightarrow \mathcal{E}_{+}^{m+n}$ is defined similarly. This gives rise to $\operatorname{Tr}_{n}: \mathcal{E}^{m+\infty} \rightarrow \mathcal{E}_{+}^{m+n}$, for all $n$, which in turn allows us to define a topological completion $\widehat{\mathcal{E}}^{m+\infty}:=\lim \mathcal{E}_{+}^{m+n}$, similarly as in [Br1, $\left.\S 2-\mathrm{d}\right]$. We call the $\operatorname{map} \operatorname{Tr}_{n}: \widehat{\mathcal{E}}^{m+\infty} \rightarrow \mathcal{E}_{+}^{m+n}$ also the truncation map. 
Lemma 4.4. Let $n \in \mathbb{N} \cup \infty$ and let $f, g \in \mathbb{Z}_{+}^{m+n}$. Then $f>g$ in the Bruhat ordering if and only if there exists $f^{0}, \cdots, f^{s} \in \mathbb{Z}_{+}^{m+n}$ such that $f=f^{0}>f^{1}>$ $\cdots>f^{s}=g$ and for each $0 \leq a<s$ there exists $i_{a}<0<j_{a}$ with $f^{a+1}=$ $\left(f^{a} \cdot \tau_{i_{a} j_{a}}\right)^{+}, f^{a}\left(i_{a}\right)>f^{a}\left(j_{a}\right)$, and $f^{a}\left(i_{a}\right) \neq f^{a}(k), \forall k>0$.

Sketch of a proof. This follows from an equivalent formulation of the Bruhat ordering in terms of the $\mathrm{wt}^{\epsilon}$ combined with an analogous proof of the reductive analog of Brundan's Lemma 3.42 in [Br1].

For $n \in \mathbb{N} \cup \infty$, a weight $f$ in $\mathbb{Z}_{+}^{m+n}$ is called $J$-typical if it is minimal in the Bruhat ordering among elements in $\mathbb{Z}_{+}^{m+n}$. The following proposition can be established in the same way as a similar statement in [Br1, Theorem 3.5]. For a finite $n$ let $\widehat{\mathcal{E}}^{m+n}:=\mathcal{E}^{m+n}$.

Proposition 4.5. Let $n \in \mathbb{N} \cup \infty$. There exists a unique anti-linear bar map - : $\widehat{\mathcal{E}}^{m+n} \rightarrow \widehat{\mathcal{E}}^{m+n}$ such that

(1) $\overline{\mathcal{K}_{f}}=\mathcal{K}_{f}$ for all $J$-typical $f \in \mathbb{Z}_{+}^{m+n}$;

(2) $\overline{X u}=\bar{X} \bar{u}$ for all $X \in \mathcal{U}$ and $u \in \widehat{\mathcal{E}}^{m+n}$;

(3) the bar map is an involution;

(4) $\overline{\mathcal{K}_{f}}=\mathcal{K}_{f}+(*)$ where $(*)$ is a (possibly infinite when $\left.n=\infty\right) \mathbb{Z}\left[q, q^{-1}\right]$-linear combination of $\mathcal{K}_{g}$ 's for $g \in \mathbb{Z}_{+}^{m+n}$ such that $g<f$ in the Bruhat ordering.

The next theorem follows from Proposition 4.5

Theorem 4.6. Let $n \in \mathbb{N} \cup \infty$. There exist unique topological bases $\left\{\mathcal{U}_{f}\right\},\left\{\mathcal{L}_{f}\right\}$, where $f \in \mathbb{Z}_{+}^{m+n}$, for $\widehat{\mathcal{E}}^{m+n}$ such that

(1) $\overline{\mathcal{U}}_{f}=\mathcal{U}_{f}$ and $\overline{\mathcal{L}}_{f}=\mathcal{L}_{f}$;

(2) $\mathcal{U}_{f} \in \mathcal{K}_{f}+\widehat{\sum}_{g \in \mathbb{Z}_{+}^{m+n}} q \mathbb{Z}[q] \mathcal{K}_{g}$ and $\mathcal{L}_{f} \in \mathcal{K}_{f}+\widehat{\sum}_{g \in \mathbb{Z}_{+}^{m+n}} q^{-1} \mathbb{Z}\left[q^{-1}\right] \mathcal{K}_{g}$;

(3) $\mathcal{U}_{f}=\mathcal{K}_{f}+(*)$ and $\mathcal{L}_{f}=\mathcal{K}_{f}+(* *)$ where $(*)$ and $(* *)$ are (possibly infinite when $n=\infty) \mathbb{Z}\left[q, q^{-1}\right]$-linear combinations of $\mathcal{K}_{g}$ 's for dominant $g<f$. For $n$ finite, $(*)$ and $(* *)$ are always finite sums.

We define $\mathfrak{u}_{g, f}(q) \in \mathbb{Z}[q], \mathfrak{l}_{g, f}(q) \in \mathbb{Z}\left[q^{-1}\right]$ for $f, g \in \mathbb{Z}_{+}^{m+n}$ by

$$
\mathcal{U}_{f}=\sum_{g \in \mathbb{Z}_{+}^{m+n}} \mathfrak{u}_{g, f}(q) \mathcal{K}_{g}, \quad \mathcal{L}_{f}=\sum_{g \in \mathbb{Z}_{+}^{m+n}} \mathfrak{l}_{g, f}(q) \mathcal{K}_{g} .
$$

Note that $\mathfrak{u}_{g, f}(q)=\mathfrak{l}_{g, f}(q)=0$ unless $g \leq f$ and $\mathfrak{u}_{f, f}(q)=\mathfrak{l}_{f, f}(q)=1$. For reasons that will become clear in Section 5.3 we will refer to these polynomials as (parabolic) Kazhdan-Lusztig polynomials.

Remark 4.7. In the same way as in Br1, (3.7)] we can show that our notation of $\mathcal{L}_{f}$ and $\mathfrak{l}_{g, f}(q)$ here is consistent with the same notation introduced in Section 4.1. Also, similar to [Br1, Lemma 3.8], we have $\mathfrak{U}_{f}=\mathcal{T}_{f \cdot w_{0}} \widehat{H}_{0}$, for $f \in \mathbb{Z}_{+}^{m+n}$.

Remark 4.8. By modifying the arguments in $\S 3-\mathrm{c}$ of Br1 we can introduce a symmetric bilinear form $\langle\cdot, \cdot\rangle$ on $\mathcal{E}^{m+n}$ such that $\left\langle\mathcal{L}_{f}, \mathcal{U}_{-g \cdot w_{0}}\right\rangle=\delta_{f, g}$, which readily implies that the matrices $\left[\mathfrak{u}_{-f \cdot w_{0},-g \cdot w_{0}}(q)\right]$ and $\left[\mathfrak{l}_{f, g}\left(q^{-1}\right)\right]$ are inverses of each other. Equivalently, we have

$$
\mathcal{K}_{f}=\sum_{g \in \mathbb{Z}_{+}^{m+n}} \mathfrak{u}_{-f \cdot w_{0},-g \cdot w_{0}}\left(q^{-1}\right) \mathcal{L}_{g}=\sum_{g \in \mathbb{Z}_{+}^{m+n}} \mathfrak{l}_{-f \cdot w_{0},-g \cdot w_{0}}\left(q^{-1}\right) \mathfrak{U}_{g}, \quad f \in \mathbb{Z}_{+}^{m+n} .
$$


4.3. Degree of $J$-atypicality. Let $n$ be finite and let $f \in \mathbb{Z}^{m+n}$ be $S_{m \mid n^{-}}$-conjugate to an element $f^{+}$in $\mathbb{Z}_{+}^{m+n}$.

For a pair of integers $(i \mid j)$, with $-m \leq i \leq-1$ and $1 \leq j \leq n$, we define the distance of $(i \mid j)$ to be $d(i \mid j):=f(i)-f(j)$. Such a pair is called an atypical pair for $f$ if the following three conditions are satisfied:

$(\mathrm{A} 1+) f(i)>f(j)$;

$(\mathrm{A} 2+)$ for every $k$ with $1 \leq k \leq n$ we have $f(i) \neq f(k)$;

$(\mathrm{A} 3+)$ for every $l$ with $-m \leq l \leq-1$ we have $f(l) \neq f(j)$.

Two atypical pairs $\left(i_{1} \mid j_{1}\right)$ and $\left(i_{2} \mid j_{2}\right)$ for $f$ are said to be disjoint if $i_{1} \neq i_{2}$ and $j_{1} \neq j_{2}$. Two subsets $A_{1}$ and $A_{2}$ of atypical pairs of $f$ are said to be disjoint if any two atypical pairs $\left(i_{1} \mid j_{1}\right) \in A_{1}$ and $\left(i_{2} \mid j_{2}\right) \in A_{2}$ are disjoint. Let $A_{f}^{+}$denote the set of all atypical pairs of $f$. For $k \geq 1$ we define the set $\Sigma_{f}^{k}$ recursively as follows:

$$
\begin{aligned}
& \Sigma_{f}^{k}:=\left\{(i \mid j) \in A_{f}^{+} \mid d(i \mid j)=k \text { and }(i \mid j) \text { is disjoint from } \bigcup_{s=1}^{k-1} \Sigma_{f}^{s}\right\}, \\
& \Sigma_{f}^{+}:=\bigcup_{k \geq 1} \Sigma_{f}^{k} .
\end{aligned}
$$

Definition 4.9. An element in $\Sigma_{f}^{+}$will be called a positive pair of $f$. The degree of $J$-atypicality of $f$, denoted by $* f$, is defined to be the cardinality of $\Sigma_{f}^{+}$.

In particular $* f \leq \min (m, n)$. By Lemma 4.4, $* f=0$ if and only if $f$ is minimal in the Bruhat ordering among elements in $\mathbb{Z}_{+}^{m+n}$; i.e. it is $J$-typical.

Remark 4.10. Our definition of $J$-atypicality was partly inspired by the definition of Leclerc and Miyachi's function $\psi$ in [LM, 2.5]. Their $\psi$ was in turn inspired by Lusztig's notion of "admissible involutions".

We denote by $A_{f}^{-}$the collection of all pairs $(i \mid j), i<0$ and $j>0$, satisfying the following three conditions:

(A1-) $f(i)<f(j)$;

(A2-) for every $k$ with $1 \leq k \leq n$ we have $f(i) \neq f(k)$;

(A3-) for every $l$ with $-m \leq l \leq-1$ we have $f(l) \neq f(j)$.

Disjointness of subsets of $A_{f}^{-}$is defined similarly. We define the set $\Sigma_{f}^{-k}$ recursively:

$$
\Sigma_{f}^{-k}:=\left\{(i \mid j) \in A_{f}^{-} \mid d(i \mid j)=-k \text { and }(i \mid j) \text { is disjoint from } \bigcup_{s=1}^{k-1} \Sigma_{f}^{-s}\right\}, \text { for } k \geq 1 \text {. }
$$

Set $\Sigma_{f}^{-}:=\bigcup_{k \geq 1} \Sigma_{f}^{-k}$. Elements in $\Sigma_{f}^{-}$will be called negative pairs of $f$.

4.4. An algorithm for canonical bases. Recall $d_{i} \in \mathbb{Z}^{m+n}$ from Subsection 2.3 . We give a procedure to reduce the degree of $J$-atypicality in a controlled manner (see Lemma 4.14).

Procedure 4.11. Let $f \in \mathbb{Z}_{+}^{m+n}$. We construct an element $h \in \mathbb{Z}_{+}^{m+n}$ and a Chevalley generator $X_{a}$ according to the following procedure.

(0) Choose $i<0$ such that $i=\max \left\{l \mid\right.$ there exists $k$ with $\left.(l \mid k) \in \Sigma_{f}^{+}\right\}$.

(1) If $i<-1$ and $f(i)-1 \neq f(i+1)$ or $i=-1$, then put $X_{a}=F_{f(i)-1}$, $h=f-d_{i}$ and the procedure stops. Otherwise go to step (2). 
(2) There exists an $s>0$ such that $f(i+1)=f(s)$ (see Remark 4.12). We let $x_{a}=E_{f(i)-1}, h=f-d_{s}$ and the procedure stops.

Remark 4.12. To justify step (2) above, we note that $(i \mid j)$ is an atypical pair for some $j>0$. Thus $f(j) \neq f(t)$ for all $t<0$, and in addition $f(i)>f(j)$, which implies that $f(i+1)=f(i)-1 \geq f(j)$. If $f(i+1) \neq f(s)$ for all $s>0$, then $(i+1 \mid j)$ is an atypical pair, and so there exists $t>0$ with $(i+1 \mid t) \in \Sigma_{f}^{+}$, contradicting the choice of $i$ in step (0).

Remark 4.13. Notice that step (1) produces an $h \in \mathbb{Z}_{+}^{m+n}$ with $\sum_{i<0} f(i)-1=$ $\sum_{i<0} h(i)$, while step (2) produces an $h \in \mathbb{Z}_{+}^{m+n}$ with $\sum_{j>0} f(j)+1=\sum_{j>0} h(j)$. It follows that a finite number of applications of the procedure reduces the degree of $J$-atypicality, and thus a finite number of repeated applications of Procedure 4.11 will produce a $J$-typical element. Procedure 4.11 may be regarded as a reductive analogue of Brundan's Procedure 3.20 in Br1].

We will write $h \rightsquigarrow f$ to denote that $h$ is obtained from $f$ via Procedure 4.11. Thus we have $h_{k} \rightsquigarrow h_{k-1} \rightsquigarrow \cdots \rightsquigarrow h_{1} \rightsquigarrow f$, with $h_{k} J$-typical. Denote the Chevalley generator corresponding to $h_{i} \rightsquigarrow h_{i-1}$ by $X_{a_{i}}$. We have the following lemma.

Lemma 4.14. Let $f \in \mathbb{Z}_{+}^{m+n}$ with $* f>0$ and let $h$ and $X_{a}$ be defined as in Procedure 4.11.

(1) If $* f=* h$, then $X_{a} \mathcal{U}_{h}=\mathcal{U}_{f}$ and $X_{a} \mathcal{K}_{h}=\mathcal{K}_{f}$.

(2) If $* f=* h+1$, then $h=f-d_{i}$, for some $i<0$. In this case we have $\mathcal{X}_{a}=F_{f(i)-1}$ and $\mathcal{X}_{a} \mathcal{U}_{h}=\mathcal{U}_{f}$ and $\mathcal{X}_{a} \mathcal{K}_{h}=\mathcal{K}_{f}+q \mathcal{K}_{h^{\prime}}$, where $h^{\prime}=$ $\left(h^{<0} \mid \tilde{e}_{f(i), f(i)-1} h^{>0}\right)$. In particular $f>h^{\prime}$.

(3) Suppose $h_{k} \rightsquigarrow h_{k-1} \rightsquigarrow \cdots \rightsquigarrow h_{1}=f$ with $h_{k} J$-typical. Then we have $\mathcal{U}_{f}=X_{a_{1}} x_{a_{2}} \ldots X_{a_{k}} \mathcal{K}_{h_{k}}$.

Proof. As usual we shall write $f=\left(f^{<0} \mid f^{>0}\right)$ and $g=\left(g^{<0} \mid g^{>0}\right)$ et cetera.

From the description of Procedure 4.11, there are the following three cases to be considered depending on the forms of $f$ and $h$, with $a+1=f(i)$. Here, $\cdots$ denotes entries different from $a$ and $a+1$.

(i) $f=(\cdots, a+1, a, \cdots \mid \cdots, a, \cdots), \quad h=(\cdots, a+1, a, \cdots \mid \cdots, a+1, \cdots)$;

(ii) $f=(\cdots, a+1, \cdots \mid \cdots), \quad h=(\cdots, a, \cdots \mid \cdots)$;

(iii) $f=(\cdots, a+1, \cdots \mid \cdots, a, \cdots), \quad h=(\cdots, a, \cdots \mid \cdots, a, \cdots)$.

We have the following description of $\Sigma_{h}^{+}$in these cases:

(i) $\Sigma_{h}^{+}=\left(\Sigma_{f}^{+} \backslash\{(i \mid k)\}\right) \cup\{(i+1 \mid k)\}$, with unique $k>0$ such that $(i \mid k) \in \Sigma_{f}^{+}$;

(ii) $\Sigma_{h}^{+}=\Sigma_{f}^{+}$;

(iii) $\Sigma_{h}^{+}=\Sigma_{f}^{+} \backslash\{(i \mid s)\}$, with unique $s>0$ such that $f(s)=a$.

Thus in (i) and (ii) above $* h=* f$, while in (iii) $* h=* f-1$.

The formulas for $\mathcal{X}_{a} \mathcal{K}_{h}$ in (1) and (2) are verified case by case using Lemma 2.2 It remains to verify the action of $\mathcal{X}_{a}$ on $\mathcal{U}_{h}$.

In (i), by Procedure 4.11, $X_{a}$ is of the form $E_{a}$. We have

$$
\mathcal{U}_{h}=\mathcal{K}_{h}+\sum_{g<h} \mathfrak{u}_{g, h}(q) \mathcal{K}_{g}, \quad \text { for } \mathfrak{u}_{g, h}(q) \in q \mathbb{Z}[q] .
$$

Observe that any $g$ such that $g<h$ is of the form

$$
g_{1}=(\cdots, a+1, a, \cdots \mid \cdots, a+1, \cdots) \text { or } g_{2}=(\cdots, a+1, \cdots \mid \cdots, a+1, a, \cdots) \text {. }
$$


One checks that

$$
x_{a} \mathcal{U}_{h}=\mathcal{K}_{f}+\sum_{g_{1}<h} \mathfrak{u}_{g_{1}, h}(q) \mathcal{K}_{g_{1}^{\prime}}+\sum_{g_{2}<h} \mathfrak{u}_{g_{2}, h}(q) \mathcal{K}_{g_{2}^{\prime}},
$$

where

$$
g_{1}^{\prime}=\left(g_{1}^{<0} \mid \tilde{e}_{a, a+1} g_{1}^{>0}\right), \quad g_{2}^{\prime}=\left(\tilde{e}_{a, a+1} g_{2}^{<0} \mid g_{2}^{>0}\right) .
$$

Note that $g_{1}^{\prime}<f$ and $g_{2}^{\prime}<f$, for $g_{1}<h$ and $g_{2}<h$. Hence $X_{a} \mathcal{U}_{h}$ is of the form $\mathcal{K}_{f}+\sum_{g<f} q \mathbb{Z}[q] \mathcal{K}_{g}$ and it is bar-invariant since

$$
\overline{x_{a} u_{h}}=\overline{x_{a}} \cdot \overline{u_{h}}=x_{a} u_{h} .
$$

Thus $\mathcal{X}_{a} \mathfrak{U}_{h}=\mathcal{U}_{f}$ by the uniqueness of the canonical basis elment $\mathfrak{U}_{f}$.

In case (ii), $X_{a}=F_{a}$. Now if $g<h$, then $g$ can be either $(\cdots, a, \cdots \mid \cdots)$ or $(\cdots \mid \cdots, a, \cdots)$. Thus $F_{a} \mathcal{K}_{g}=\mathcal{K}_{g^{\prime}}$, where $g^{\prime}$ is either $(\cdots, a+1, \cdots \mid \cdots)$ or $(\cdots \mid \cdots, a+1, \cdots)$. In either case $g^{\prime}<f$. From a similar argument as in case (i), we conclude that $X_{a} \mathcal{U}_{h}=\mathcal{U}_{f}$. In case (iii), $X_{a}=F_{a}$ and if $g<h$, then $g$ is of the same form as $h$. Now this case is straightforward to check, thus completing the proofs of (1) and (2). By (1) and (2), $x_{a_{1}} x_{a_{2}} \ldots \mathcal{X}_{a_{k}} \mathcal{K}_{h_{k}}$ is a bar-invariant element of the form $\mathcal{K}_{f}+\sum_{g<f} q \mathbb{Z}[q] \mathcal{K}_{g}$, hence equals $\mathcal{U}_{f}$, proving (3).

Example 4.15. Consider $f=(2,1,0 \mid 3,0,-2)$ with $* f=1$. We will use Procedure 4.11 to compute $\mathcal{U}_{f}$. We have the following sequence:

$(2,1,-2 \mid 3,1,-2) \rightsquigarrow(2,1,-1 \mid 3,1,-2) \rightsquigarrow(2,1,0 \mid 3,1,-2) \rightsquigarrow(2,1,0 \mid 3,0,-2)$,

Hence, $\mathcal{U}_{f}=E_{0} F_{-1} F_{-2} \mathcal{K}_{(2,1,-2 \mid 3,1,-2)}=\mathcal{K}_{(2,1,0 \mid 3,0,-2)}+q \mathcal{K}_{(2,0,-2 \mid 3,1,0)}$.

Let $\Sigma=\left\{\left(i_{1} \mid j_{1}\right), \cdots,\left(i_{k} \mid j_{k}\right)\right\}$ be a subset of $\{-m, \cdots,-1\} \times\{1, \cdots, n\}$ such that all the $i_{s}$ 's and also all the $j_{s}$ 's are distinct. Let $f \in \mathbb{Z}_{+}^{m+n}$. Define an element $f_{\Sigma} \in \mathbb{Z}_{+}^{m+n}$ obtained from $f$ as follows. It is enough to specify the values of $f_{\Sigma}^{<0}$ and $f_{\Sigma}^{>0}$. The values of $f_{\Sigma}^{<0}$ are obtained from the values of $f^{<0}$ with $f\left(j_{s}\right)$ replacing $f\left(i_{s}\right)$, and the values of $f_{\Sigma}^{>0}$ are obtained from the values of $f^{>0}$ with $f\left(i_{s}\right)$ replacing $f\left(j_{s}\right)$, for all $s=1, \cdots, k$.

Theorem 4.16. Let $f \in \mathbb{Z}_{+}^{m+n}$. We have

$$
\mathcal{U}_{f}=\sum_{\Sigma \subseteq \Sigma_{f}^{+}} q^{|\Sigma|} \mathcal{K}_{f_{\Sigma}}
$$

where the summation is over all subsets $\Sigma$ of $\Sigma_{f}^{+}$.

Proof. If $f$ itself is $J$-typical, then $\mathcal{K}_{f}=\mathcal{U}_{f}$ and the theorem is true in this case, since $\Sigma_{f}^{+}=\emptyset$.

Suppose that $* f>0$. Using the notation of Procedure 4.11 and Lemma 4.14 we have $\mathfrak{U}_{f}=X_{a} \mathfrak{U}_{h}$. We may assume by induction based on Procedure 4.11 that $\mathcal{U}_{h}$ is of the form $\mathcal{U}_{h}=\sum_{\Sigma \subseteq \Sigma_{h}^{+}} q^{|\Sigma|} \mathcal{K}_{h_{\Sigma}}$. There are three possibilities for $\mathcal{X}_{a}$, where $a+1=f(i)$.

In the first case, $X_{a}=E_{a}$ and the $J$-atypicality is not changed. Here we must have $f=(\cdots, a+1, a, \cdots \mid \cdots, a, \cdots)$ and $h=(\cdots, a+1, a, \cdots \mid \cdots, a+1, \cdots)$. In this case if $(i \mid k) \in \Sigma_{f}^{+}$, then $\Sigma_{h}^{+}=\left(\Sigma_{f}^{+} \backslash\{(i \mid k)\}\right) \cup\{(i+1, k)\}$. Now one checks that

$$
\mathcal{U}_{f}=E_{a} \mathcal{U}_{h}=\sum_{\Sigma \subseteq \Sigma_{h}^{+}} q^{|\Sigma|} E_{a} \mathcal{K}_{h_{\Sigma}}=\sum_{\Sigma \subseteq \Sigma_{f}^{+}} q^{|\Sigma|} \mathcal{K}_{f_{\Sigma}}
$$


In the second case, $X_{a}=F_{a}$ and the $J$-atypicality is not changed. In this case, $f=(\cdots, a+1, \cdots \mid \cdots)$ and $h$ is of the form $(\cdots, a, \cdots \mid \cdots)$, and we have $\Sigma_{h}^{+}=\Sigma_{f}^{+}$. Thus

$$
\mathcal{U}_{f}=F_{a} \mathcal{U}_{h}=\sum_{\Sigma \subseteq \Sigma_{h}^{+}} q^{|\Sigma|} F_{a} \mathcal{K}_{h_{\Sigma}}=\sum_{\Sigma \subseteq \Sigma_{f}^{+}} q^{|\Sigma|} \mathcal{K}_{f_{\Sigma}}
$$

Finally suppose that $X_{a}=F_{a}$ and the atypicality is changed. In this case, $f=(\cdots, a+1, \cdots \mid \cdots, a, \cdots)$ and $h=(\cdots, a, \cdots \mid \cdots, a, \cdots)$. Let $s>0$ be such that $f(s)=a$. Then $\Sigma_{h}^{+}=\Sigma_{f}^{+} \backslash\{(i \mid s)\}$. Thus any $h_{\Sigma}$ is of the form $(\cdots, a, \cdots \mid \cdots, a, \cdots)$ and we have

$$
\begin{aligned}
\mathcal{U}_{f}=F_{a} \mathcal{U}_{h} & =\sum_{\Sigma \subseteq \Sigma_{h}^{+}} q^{|\Sigma|} F_{a} \mathcal{K}_{h_{\Sigma}} \\
& =\sum_{\Sigma \subseteq \Sigma_{h}^{+}} q^{|\Sigma|} \mathcal{K}_{f_{\Sigma}}+\sum_{\Sigma \subseteq \Sigma_{h}^{+}} q^{|\Sigma|+1} \mathcal{K}_{f_{\Sigma \cup\{(i \mid s)\}}}=\sum_{\Sigma \subseteq \Sigma_{f}^{+}} q^{|\Sigma|} \mathcal{K}_{f_{\Sigma}} .
\end{aligned}
$$

This completes the proof.

Remark 4.17. Theorem 4.16 is a generalization of Theorem 3 of [LM], where the canonical basis of the irreducible $\mathcal{U}$-module generated by the vacuum vector of the Fock space was computed. The same combinatorics was used in Lec to describe the composition factors of tensor products of evaluation modules for quantum affine algebras.

Let $f \in \mathbb{Z}_{+}^{m+n}$ and let $w_{0}$ be the longest element in $S_{m \mid n}$. From the definitions the negative pairs of $f$ are precisely the positive pairs of $-f \cdot w_{0}$ via the correspondence

$$
(i \mid j) \leftrightarrow(-m-i-1 \mid n-j+1) .
$$

We have the following consequence of Remark 4.8 and Theorem 4.16

Corollary 4.18. Let $f \in \mathbb{Z}_{+}^{m+n}$. We have

$$
\mathcal{K}_{f}=\sum_{g} q^{-|\Sigma|} \mathcal{L}_{g}
$$

summed over all $g \in \mathbb{Z}_{+}^{m+n}$ such that there exists $\Sigma \subseteq \Sigma_{g}^{-}$with $g_{\Sigma}=f$.

4.5. The operators $\mathbb{L}$ and $\mathbb{R}$. Theorem 4.16 computes $\mathfrak{u}_{g, f}$, and Corollary 4.18 computes the polynomials $\mathfrak{l}_{g, f}$ upon inversion. In the remainder of this section we will introduce some combinatorics and establish the reductive counterparts of Theorem 3.34 and Corollary 3.36 of Br1] that allow us to describe this inversion explicitly. We start with the following lemma, which follows from the definition.

Lemma 4.19. Let $f \in \mathbb{Z}^{m+n}$ be $S_{m \mid n}$-conjugate to an element in $\mathbb{Z}_{+}^{m+n}$ and suppose that $(i \mid j)$ is an atypical pair of $f$. Then $(i \mid j)$ and $\Sigma_{f}^{+}$are not disjoint.

Corollary 4.20. Let $f \in \mathbb{Z}^{m+n}$ be $S_{m \mid n}$-conjugate to an element in $\mathbb{Z}_{+}^{m+n}$, and suppose that $(i \mid j)$ is an atypical pair of $f$. Then there exists $i^{\prime}<0$ with $f\left(i^{\prime}\right) \leq f(i)$ such that $\left(i^{\prime} \mid j\right) \in \Sigma_{f}^{+}$or there exists $j^{\prime}>0$ with $f\left(j^{\prime}\right) \geq f(j)$ such that $\left(i \mid j^{\prime}\right) \in \Sigma_{f}^{+}$.

Next we define the reductive analogue of the L-operators and R-operators. Let $f \in \mathbb{Z}^{m+n}$ be $S_{m \mid n^{-c o n j u g a t e}}$ to an element in $\mathbb{Z}_{+}^{m+n}$, with positive pairs $\Sigma_{f}^{+}$and 
negative pairs $\Sigma_{f}^{-}$. Recalling $\tau_{i j} \in S_{m+n}$ from Subsection 2.2 we define for each $i=-m, \cdots,-1$

$$
\begin{aligned}
& \mathbb{L}_{i}(f):= \begin{cases}f \cdot \tau_{i j}, & \text { if there exists } j \text { with }(i \mid j) \in \Sigma_{f}^{+}, \\
\emptyset, & \text { otherwise. }\end{cases} \\
& \mathbb{R}_{i}(f):= \begin{cases}f \cdot \tau_{i j}, & \text { if there exists } j \text { with }(i \mid j) \in \Sigma_{f}^{-}, \\
\emptyset, & \text { otherwise. }\end{cases}
\end{aligned}
$$

Suppose now that $f \in \mathbb{Z}_{+}^{m+n}$. For $\theta=\left(\theta_{-m}, \cdots, \theta_{-1}\right) \in \mathbb{N}^{m}$ we define

$$
\begin{aligned}
& \mathbb{L}_{\theta}(f):=\left(\mathbb{L}_{-1}^{\theta_{-1}} \circ \mathbb{L}_{-2}^{\theta_{-2}} \circ \cdots \circ \mathbb{L}_{-m}^{\theta_{-m}}(f)\right)^{+}, \quad \mathbb{L}_{\theta}^{\prime}(f):=\left(\mathbb{L}_{-m}^{\theta_{-m}} \circ \cdots \circ \mathbb{L}_{-1}^{\theta_{-1}}(f)\right)^{+}, \\
& \mathbb{R}_{\theta}^{\prime}(f):=\left(\mathbb{R}_{-1}^{\theta_{-1}} \circ \mathbb{R}_{-2}^{\theta_{-2}} \circ \cdots \circ \mathbb{R}_{-m}^{\theta_{-m}}(f)\right)^{+}, \quad \mathbb{R}_{\theta}(f):=\left(\mathbb{R}_{-m}^{\theta_{-m}} \circ \cdots \circ \mathbb{R}_{-1}^{\theta_{-1}}(f)\right)^{+} .
\end{aligned}
$$

Write $|\theta|:=\sum_{i=-m}^{-1} \theta_{i}$. Let $\Sigma_{f}^{+}=\left\{\left(i_{1} \mid j_{1}\right), \cdots,\left(i_{k} \mid j_{k}\right)\right\}$, where $i_{1}<i_{2}<\cdots<i_{k}<$

0 . We shall denote by $\mathbb{L}(f)$ (or $f^{\mathbb{L}}$ ) the expression

$$
\mathbb{L}(f):=\left(\mathbb{L}_{i_{k}} \circ \mathbb{L}_{i_{k-1}} \cdots \circ \mathbb{L}_{i_{1}}(f)\right)^{+} .
$$

Similarly, if $\Sigma_{f}^{-}=\left\{\left(i_{1} \mid j_{1}\right), \cdots,\left(i_{k} \mid j_{k}\right)\right\}$, with $i_{1}<i_{2}<\cdots<i_{k}<0$, we shall write $\mathbb{R}(f)\left(\right.$ or $\left.f^{\mathbb{R}}\right)$ for the expression

$$
\mathbb{R}(f):=\left(\mathbb{R}_{i_{1}} \circ \mathbb{R}_{i_{2}} \cdots \circ \mathbb{R}_{i_{k}}(f)\right)^{+} .
$$

The following follows from the definitions.

Lemma 4.21. Let $f \in \mathbb{Z}_{+}^{m+n}$ and let $w_{0}$ be the longest element in $S_{m \mid n}$. For $\theta=\left(\theta_{-m}, \cdots, \theta_{-1}\right) \in \mathbb{N}^{m}$ let $\varphi=\left(\theta_{-1}, \cdots, \theta_{-m}\right)$. We have

$$
-\mathbb{L}_{\theta}\left(-f \cdot w_{0}\right) \cdot w_{0}=\mathbb{R}_{\varphi}(f), \quad-\mathbb{L}_{\theta}^{\prime}\left(-f \cdot w_{0}\right) \cdot w_{0}=\mathbb{R}_{\varphi}^{\prime}(f) .
$$

4.6. Transition matrices between monomial and canonical bases in $\mathcal{E}^{m+n}$. We start with several simple lemmas whose proofs are straightforward.

Lemma 4.22. Let $f \in \mathbb{Z}^{m+n}$ be $S_{m \mid n^{-}}$conjugate to an element in $\mathbb{Z}_{+}^{m+n}$. Suppose that $\left(i_{0} \mid j_{0}\right) \in \Sigma_{f}^{+}$.

(i) If there exists $b>0$ such that $f(b) \notin\{f(i) \mid i<0\}$ and $f\left(i_{0}\right)>f(b)>f\left(j_{0}\right)$, then there exists $a<0$ with $f\left(i_{0}\right)>f(a)>f(b)$ and $(a \mid b) \in \Sigma_{f}^{+}$.

(ii) If there exists $a<0$ with $f(a) \notin\{f(j) \mid j>0\}$ such that $f\left(i_{0}\right)>f(a)>$ $f\left(j_{0}\right)$, then there exists $b>0$ with $f(a)>f(b)>f\left(j_{0}\right)$ and $(a \mid b) \in \Sigma_{f}^{+}$.

Lemma 4.23. Let $f \in \mathbb{Z}^{m+n}$ be $S_{m \mid n}$-conjugate to an element in $\mathbb{Z}_{+}^{m+n}$, and let $(i \mid k),(j \mid l) \in \Sigma_{f}^{+}$with $f(i)>f(j)$. Then $(j \mid l) \in \Sigma_{\mathbb{L}_{i}(f)}^{+}$.

Lemma 4.24. Let $f \in \mathbb{Z}^{m+n}$ be $S_{m \mid n}$-conjugate to an element in $\mathbb{Z}_{+}^{m+n}$, and let $(i \mid k) \in \Sigma_{f}^{+}$. Suppose $i<0, j<0$ and $f(i)>f(j)$, and there is no $l>0$ such that $(j \mid l) \in \Sigma_{f}^{+}$. Then there is no $l$ such that $(j \mid l) \in \Sigma_{\mathbb{L}_{i}(f)}^{+}$.

Proof. The lemma is obvious if there exists $b>0$ such that $f(j)=f(b)$. Hence we may assume that this is not the case.

Suppose there exists an $l>0$ such that $f(l) \neq f(c)$, for every $c<0$, and $f(j)>f(l)$. Then there exists an $a<0$ with $f(j)>f(a)>f(l)$ and $(a \mid l) \in \Sigma_{f}^{+}$. But then by Lemma 4.23 we have $(a \mid l) \in \Sigma_{\mathbb{L}_{i}(f)}^{+}$, and hence $(j \mid l) \notin \Sigma_{\mathbb{L}_{i}(f)}^{+}$. 
Theorem 4.25. Let $f \in \mathbb{Z}_{+}^{m+n}$. We have

(1) $\mathcal{U}_{f}=\sum_{\theta \in\{0,1\}^{m}} q^{|\theta|} \mathcal{K}_{\mathbb{L}_{\theta}(f)}$;

(2) $\mathcal{K}_{f}=\sum_{\theta \in \mathbb{N}^{m}}(-q)^{|\theta|} \mathcal{U}_{\mathbb{L}_{\theta}^{\prime}(f)}$.

Proof. Part (1) follows from Theorem 4.16 and Lemmas 4.23 and 4.24 .

Part (2) can be proved using (1) following the strategy of the proof of Theorem 3.34 (ii) in $\mathrm{Br} 1$ as follows. We let $M^{m+n}$ be the free $\mathbb{Z}\left[q, q^{-1}\right]$-module with basis $\{[f]\}$, where the $f$ 's are all the $S_{m \mid n}$-conjugates of elements in $\mathbb{Z}_{+}^{m+n}$. We define linear maps $\mathcal{U}, \mathcal{K}: M^{m+n} \rightarrow \mathcal{E}^{m+n}$ by $\mathcal{K}([f])=\mathcal{K}_{f+}, \mathcal{U}([f])=\mathcal{U}_{f+}$. Let $\mathcal{K}^{-1}, \mathcal{U}^{-1}$ denote the right inverse functions of $\mathcal{K}$ and $\mathcal{U}$, respectively, given by $\mathcal{K}^{-1}\left(\mathcal{K}_{f}\right)=$ $[f], \mathcal{U}^{-1}\left(\mathcal{U}_{f}\right)=[f]$. Now for $-m \leq i \leq-1$ define $\lambda_{i}: M^{m+n} \rightarrow M^{m+n}$ by

$$
\lambda_{i}([f]):=\left\{\begin{array}{l}
{\left[f \cdot \tau_{i j}\right], \quad \text { if there exists } j \text { with }(i \mid j) \in \Sigma_{f}^{+},} \\
0, \quad \text { otherwise. }
\end{array}\right.
$$

Consider the maps

$$
\begin{aligned}
& \mathcal{P}:=\mathcal{K} \circ\left(\left(1+q \lambda_{-1}\right) \cdots\left(1+q \lambda_{-m}\right)\right) \circ \mathcal{U}^{-1}: \mathcal{E}^{m+n} \rightarrow \mathcal{E}^{m+n} \\
& \mathcal{Q}:=\mathcal{U} \circ\left(\frac{1}{1+q \lambda_{-m}} \cdots \frac{1}{1+q \lambda_{-1}}\right) \circ \mathcal{K}^{-1}: \mathcal{E}^{m+n} \rightarrow \mathcal{E}^{m+n}
\end{aligned}
$$

Note that every $\lambda_{i}$ is nilpotent, so the expression $1+q \lambda_{i}$ is invertible.

Now (1) says that $\mathcal{P}\left(\mathcal{U}_{f}\right)=\mathcal{U}_{f}$ and hence $\mathcal{P}$ is the identity map. Part (2) is equivalent to saying that $\mathcal{Q}$ is the identity map. So it suffices to show that $\mathcal{P} \circ \mathcal{Q}$ is the identity map, which amounts to removing $\mathcal{U}^{-1} \circ \mathcal{U}$ in $\mathcal{P} \circ \mathcal{Q}$. Now this would follow, once we can show that, given $f \in \mathbb{Z}_{+}^{m+n}$ and a nonzero summand $[g]$ of $\frac{1}{1+q \lambda_{-m}} \cdots \frac{1}{1+q \lambda_{-1}}[f]$,

$$
\mathcal{K} \circ\left(1+q \lambda_{-1}\right) \cdots\left(1+q \lambda_{-m}\right)[g]=\mathcal{K} \circ\left(1+q \lambda_{-1}\right) \cdots\left(1+q \lambda_{-m}\right)\left[g^{+}\right] .
$$

We make the following three observations for $[g]$ a summand of $\frac{1}{1+q \lambda_{-m}} \cdots \frac{1}{1+q \lambda_{-1}}[f]$, for $f \in \mathbb{Z}_{+}^{m+n}$ :

(i) If $g$ is of the form $(\cdots g(i) \cdots g(j) \cdots \mid \cdots)$ with $g(i)<g(j), i<j$ and $(i \mid k),(j \mid l) \in \Sigma_{g}^{+}$, for some $k, l>0$, then $g(l)>g(i)$.

(ii) For $g$ having the form of (i), $\mathbb{L}_{i} \mathbb{L}_{j}(g)$ and $\mathbb{L}_{j} \mathbb{L}_{i}(g)$ are $S_{m \mid n}$-conjugates.

(iii) For $g$ of the form (i) and $-m \leq i_{1}<i_{2}<\cdots<i_{k}<j<0$ with $i \neq i_{s}$ for $s=1, \cdots, k$, the element $\mathbb{L}_{i_{k}} \cdots \mathbb{L}_{i_{1}}(g)$ is also of the form (i).

Now let $g$ be such a summand. For $-m \leq i_{1}<i_{2}<\cdots<i_{k}<0$ consider $h=\mathbb{L}_{i_{k}} \cdots \mathbb{L}_{i_{1}}(g)$. Of course there exist distinct $-m \leq j_{1}, \cdots, j_{k}<0$ such that $\mathbb{L}_{j_{k}} \cdots \mathbb{L}_{j_{1}}\left(g^{+}\right)$is $S_{m \mid n}$-conjugate to $h$. In order to establish (4.5) we only need to show that if we rearrange the $j_{1}, \cdots, j_{k}$ in decreasing order and apply the corresponding $\mathbb{L}$-operators to $g^{+}$we get an element that is also $S_{m \mid n}$-conjugate to $h$. This follows from (ii) and (iii). Below is an example to illustrate this. Suppose

$$
\begin{aligned}
& g=\left(\cdots, g\left(i_{1}\right), \cdots, g\left(i_{2}\right), \cdots, g\left(i_{3}\right) \cdots \mid \cdots\right), \\
& g^{+}=\left(\cdots, g\left(i_{3}\right), \cdots, g\left(i_{2}\right), \cdots, g\left(i_{1}\right), \cdots \mid \cdots\right),
\end{aligned}
$$

with $g\left(i_{s}\right)=g^{+}\left(j_{s}\right)$ with $s=1,2,3$. We write $h \sim h^{\prime}$ if $h$ and $h^{\prime}$ are $S_{m \mid n^{-}}$ conjugates. We have by (ii) and (iii),

$$
\mathbb{L}_{i_{3}} \mathbb{L}_{i_{2}} \mathbb{L}_{i_{1}} g \sim \mathbb{L}_{i_{3}} \mathbb{L}_{i_{1}} \mathbb{L}_{i_{2}} g \sim \mathbb{L}_{i_{1}} \mathbb{L}_{i_{3}} \mathbb{L}_{i_{2}} g \sim \mathbb{L}_{i_{1}} \mathbb{L}_{i_{2}} \mathbb{L}_{i_{3}} g
$$

But $\mathbb{L}_{i_{1}} \mathbb{L}_{i_{2}} \mathbb{L}_{i_{3}} g$ is $S_{m \mid n^{-}}$-conjugate to $\mathbb{L}_{j_{3}} \mathbb{L}_{j_{2}} \mathbb{L}_{j_{1}} g^{+}$. 
Remark 4.26. The formula for $\mathcal{U}_{f}$ in Theorem 4.25 (or in Theorem 4.16) implies that $\mathcal{U}_{f}$ is a sum of precisely $2^{* f}$ monomial basis elements.

\subsection{Transition matrices with dual canonical basis in $\mathcal{E}^{m+n}$.}

Corollary 4.27. Let $f \in \mathbb{Z}_{+}^{m+n}$. We have

(1) $\mathcal{K}_{f}=\sum_{\mathbb{R}_{\theta}(g)=f} q^{-|\theta|} \mathcal{L}_{g}$,

(2) $\mathcal{L}_{f}=\sum_{\mathbb{R}_{\theta}^{\prime}(g)=f}(-q)^{-|\theta|} \mathcal{K}_{g}$,

where the summation in (1) is over all $g \in \mathbb{Z}_{+}^{m+n}$ such that there exists $\theta \in\{0,1\}^{m}$ with $\mathbb{R}_{\theta}(g)=f$, and the summation in (2) is over all $g \in \mathbb{Z}_{+}^{m+n}$ and $\theta \in \mathbb{N}^{m}$ such that $\mathbb{R}_{\theta}^{\prime}(g)=f$.

Proof. In order to establish part (1) we need to compute $\mathfrak{u}_{-f w_{0},-g w_{0}}\left(q^{-1}\right)$ according to Remark 4.8. By Theorem 4.25 (1) it is equal to $q^{-|\theta|}$ if $-f \cdot w_{0}=\mathbb{L}_{\theta}\left(-g \cdot w_{0}\right)$, where $\theta \in\{0,1\}^{m}$, which is equivalent to saying that $f=-\mathbb{L}_{\theta}\left(-g \cdot w_{0}\right) \cdot w_{0}$. Now by Lemma 4.21 we have $-\mathbb{L}_{\theta}\left(-g \cdot w_{0}\right) \cdot w_{0}=\mathbb{R}_{\varphi}(g)$, where we recall that the expression $\varphi$ means $\theta$ in reversed order. Thus we obtain $\mathbb{R}_{\varphi}(g)=f$, as desired.

Part (2) is proved in a similar way using the second identity in Remark 4.8 together with Theorem 4.25 (2) and Lemma 4.21.

Corollary 4.28. Both $\mathfrak{u}_{g, f}(q)$ and $\mathfrak{l}_{g, f}\left(-q^{-1}\right)$ are polynomials in $q$ with positive integer coefficients. More explicitly,

(1) $\mathfrak{u}_{g, f}(q)=q^{|\theta|}$ if $g=\mathbb{L}_{\theta}(f)$ for some $\theta \in\{0,1\}^{m}$, and $\mathfrak{u}_{g, f}(q)=0$ otherwise;

(2) $\mathfrak{l}_{g, f}\left(-q^{-1}\right)=\sum_{\theta} q^{|\theta|}$, where the sum is over all $\theta \in \mathbb{N}^{m}$ with $\mathbb{R}_{\theta}^{\prime}(g)=f$.

4.8. Transition matrices for bases in $\widehat{\mathcal{E}}^{m+\infty}$.

Proposition 4.29. The truncation map $\operatorname{Tr}_{n+1, n}: \mathcal{E}_{+}^{m+n+1} \rightarrow \mathcal{E}_{+}^{m+n}$ commutes with the bar-involution.

Proof. The proposition can be proved by an almost identical argument as in Proposition 2.8. We will not give the details except for pointing out that we now work over the space $\mathcal{E}^{m+n} \otimes \mathbb{V}$ and we have the following counterparts of (2.7) and (2.8):

$$
\begin{aligned}
\Theta^{(m+n, 1)}= & 1 \otimes 1+\left(q-q^{-1}\right) \sum_{a, b: a<b} \tilde{E}_{a b} \otimes e_{b a}, \\
\overline{\mathcal{K}_{f(n)} \otimes v_{f(n+1)}=} & \overline{\mathcal{K}_{f(n)}} \otimes v_{f(n+1)} \\
& +\left(q-q^{-1}\right) \sum_{b>f(n+1)} \tilde{E}_{f(n+1), b} \overline{\mathcal{K}_{f(n)}} \otimes v_{b} .
\end{aligned}
$$

Similarly as in Subsection 2.6. Proposition 4.29 implies the following.

Corollary 4.30. (1) $\operatorname{Tr}_{n+1, n}$ sends $\mathcal{U}_{f}$ (respectively $\mathcal{L}_{f}$ ) to $\mathcal{U}_{f^{(n)}}$ (respectively $\left.\mathcal{L}_{f(n)}\right)$ if $f(n+1)=-n$ and to 0 otherwise.

(2) For $f, g \in \mathbb{Z}_{++}^{m+(n+1)}$ such that $f(n+1)=g(n+1)=-n$, we have

$$
\mathfrak{u}_{g, f}(q)=\mathfrak{u}_{g^{(n)}, f^{(n)}}(q), \quad \mathfrak{l}_{g, f}(q)=\mathfrak{l}_{g^{(n)}, f^{(n)}}(q) .
$$

Remark 4.31. By Proposition 4.29 and stabilizing its finite $n$ counterparts (Theorem 4.25. Corollary 4.27) we see that Theorem 4.25 and Corollary 4.27 remain to be valid for $n=\infty$. 


\section{Representation theORY of $\mathfrak{g l}(m+n)$}

5.1. The categories $\mathcal{O}_{m+n}, \mathcal{O}_{m+n}^{+}$and $\mathcal{O}_{m+n}^{++}$. Let $n \in \mathbb{N}$. We shall think of $\mathfrak{g l}(m+n)$ as the Lie algebra of complex matrices whose rows and columns are parameterized by $I(m \mid n)$. We denote by $\mathfrak{h}_{c}$ (respectively $\mathfrak{b}_{c}$ ) the standard Cartan (respectively Borel) subalgebra of $\mathfrak{g l}(m+n)$, which consists of all diagonal (respectively upper triangular) matrices. Let $\left\{\delta_{i}^{\prime}, i \in I(m \mid n)\right\}$ be the basis of $\mathfrak{h}_{c}^{*}$ dual to $\left\{e_{i i}, i \in I(m \mid n)\right\}$. Set $\mathfrak{g}_{0}=\mathfrak{g l}(m) \oplus \mathfrak{g l}(n)$. Let $\mathfrak{q}$ be a parabolic subalgebra $\mathfrak{g}_{0}+\mathfrak{b}_{c}$. Similarly as in Section 3 the Lie algebra $\mathfrak{g l}(m+\infty)$ is defined as $\lim _{\vec{n}} \mathfrak{g l}(m+n)$.

Define the symmetric bilinear form $(\cdot \mid \cdot)_{c}$ on $\mathfrak{h}_{c}^{*}$ by

$$
\left(\delta_{i}^{\prime} \mid \delta_{j}^{\prime}\right)_{c}=\delta_{i j}, \quad i, j \in I(m \mid n),
$$

and let

$$
\rho_{c}=-\sum_{i=-m}^{-1} i \delta_{i}^{\prime}+\sum_{j=1}^{n}(1-j) \delta_{j}^{\prime}
$$

Let $X_{m+n}$ be the set of integral weights $\lambda=\sum_{i \in I(m \mid n)} \lambda_{i} \delta_{i}^{\prime}, \lambda_{i} \in \mathbb{Z}$, let $X_{m+n}^{+}$be the set of all $\lambda \in X_{m+n}$ such that $\lambda_{-m} \geq \cdots \geq \lambda_{-1}, \lambda_{1} \geq \cdots \geq \lambda_{n}$ (such a weight will be referred to as dominant), and let $X_{m+n}^{++}$be the set of all $\lambda \in X_{m+n}^{+}$such that $\lambda_{n} \geq 0$. We may regard an element $\lambda$ in $X_{m+n}^{++}$as an element in $X_{m+n+1}^{++}$by letting $\lambda_{n+1}=0$. Analogously we denote by $X_{m+\infty}^{+}$the set of all dominant integral weights $\lambda=\sum_{i \in I(m \mid \infty)} \lambda_{i} \delta_{i}^{\prime} \in X_{m+\infty}$ such that $\lambda_{-m} \geq \cdots \geq \lambda_{-1}, \lambda_{1} \geq \lambda_{2} \geq \cdots$, and $\lambda_{i}=0$ for $i \gg 0$.

Given $\lambda \in X_{m+n}^{+}, n \in \mathbb{N} \cup \infty$, we denote the Verma and generalized Verma modules by

$$
\mathcal{V}_{n}(\lambda):=U(\mathfrak{g l}(m+n)) \otimes_{U\left(\mathfrak{b}_{c}\right)} \mathbb{C}_{\lambda}, \quad \mathcal{K}_{n}(\lambda):=U(\mathfrak{g l}(m+n)) \otimes_{U(\mathfrak{q})} L_{n}^{0}(\lambda),
$$

respectively. Here $L_{n}^{0}(\lambda)$ is extended trivially to a $\mathfrak{q}$-module, and $\mathbb{C}_{\lambda}$ is the standard one-dimensional $\mathfrak{h}_{c}$-module extended trivially to a $\mathfrak{b}_{c}$-module. Let $\mathcal{L}_{n}(\lambda)$ be the irreducible $\mathfrak{g r}(m+n)$-module of highest weight $\lambda$.

Let $n \in \mathbb{N} \cup \infty$. Define a bijection

$$
X_{m+n} \longrightarrow \mathbb{Z}^{m+n}, \quad \lambda \mapsto f_{\lambda},
$$

where $f_{\lambda} \in \mathbb{Z}^{m+n}$ is given by $f_{\lambda}(i)=\left(\lambda+\rho_{c} \mid \delta_{i}^{\prime}\right)_{c}$ for $i \in I(m \mid n)$. This map induces bijections $X_{m+n}^{+} \rightarrow \mathbb{Z}_{+}^{m+n}$ (for $n$ possibly infinite) and $X_{m+n}^{++} \rightarrow \mathbb{Z}_{++}^{m+n}$ (for finite $n)$. Notions, such as Bruhat ordering and degree of $J$-atypicality etc., are defined on elements of $X_{m+n}$ in a way that is compatible with the ones defined on elements of $\mathbb{Z}^{m+n}$.

Let $n \in \mathbb{N}$. Denote by $\mathcal{O}_{m+n}$ the category of finitely generated $\mathfrak{g l}(m+n)$-modules $M$ that are locally finite over $\mathfrak{b}_{c}$, semisimple over $\mathfrak{h}_{c}$ and

$$
M=\bigoplus_{\gamma \in X_{m+n}} M_{\gamma},
$$

where as usual $M_{\gamma}$ denotes the $\gamma$-weight space of $M$ with respect to $\mathfrak{h}_{c}$. The objects in $\mathcal{O}_{m+n}$ include the Verma module $\mathcal{V}_{n}(\lambda)$ and the irreducible module $\mathcal{L}_{n}(\lambda)$ for $\lambda \in X_{m+n}$. Denote by $\mathcal{O}_{m+n}^{+}$the full subcategory of finitely generated $\mathfrak{g l}(m+n)$ modules $M$ which are locally finite over $\mathfrak{q}$. The generalized Verma module $\mathcal{K}_{n}(\lambda)$ and the irreducible module $\mathcal{L}_{n}(\lambda)$ for $\lambda \in X_{m+n}^{+}$belong to $\mathcal{O}_{m+n}^{+}$. Denote by 
$\mathcal{O}_{m+n}^{++}$the full subcategory of $\mathcal{O}_{m+n}^{+}$which consists of $\mathfrak{g l}(m+n)$-modules $M$ whose composition factors are isomorphic to $\mathcal{L}_{n}(\lambda)$ with $\lambda \in X_{m+n}^{++}$.

Given $M \in \mathcal{O}_{m+n}^{+}$, we endow the restricted dual $M^{*}$ with the usual $\mathfrak{g l}(m+n)$ module structure. Further twisting the $\mathfrak{g l}(m+n)$-action on $M^{*}$ by the automorphism given by the negative transpose of $\mathfrak{g l}(m+n)$, we obtain another $\mathfrak{g}$-module denoted by $M^{\tau}$. For $M$ with finite-dimensional weight spaces we have $\left(M^{\tau}\right)^{\tau} \cong M$.

The category $\mathcal{O}_{m+\infty}^{++}$consists of finitely generated $\mathfrak{g l}(m+\infty)$-modules that are locally finite over $\mathfrak{q} \cap \mathfrak{g l}(m+N)$, for every $N$, and such that the composition factors are of the form $\mathcal{L}(\lambda), \lambda \in X_{m+\infty}^{+}$.

A $\mathfrak{g l}(m+n)$-module $M$ is said to have a generalized Verma flag if there exists a filtration of $\mathfrak{g l}(m+n)$-modules $0=M_{0} \subseteq M_{1} \subseteq \cdots \subseteq M_{r}=M$ such that $M_{i} / M_{i-1}$ is isomorphic to some generalized Verma module for each $i$. Denote by $\left(M: \mathcal{K}_{n}(\lambda)\right)$ the number of subquotients of $M$ which are isomorphic to $\mathcal{K}_{n}(\lambda)$.

Tilting modules in $\mathcal{O}_{m+n}$ and $\mathcal{O}_{m+n}^{+}$are defined similarly as before; cf. Definition 3.10. The role of standard modules there is played by Kac modules, while here it is played by generalized Verma modules. For a finite $n$, the notion of the tilting modules in $\mathcal{O}_{m+n}$ and $\mathcal{O}_{m+n}^{+}$first appeared in $\mathrm{CoI}$ in a somewhat different formulation (see [So3] for generalizations based on the work of Ringel and others; also cf. [Br2]).

The character formula of the tilting module $\mathcal{T}_{n}(\mu)$ in $\mathcal{O}_{m+n}$ is given as follows [So3] (also see [Br2]):

$$
\left(\mathcal{T}_{n}(\mu): \mathcal{V}_{n}(\nu)\right)=\left[\mathcal{V}_{n}\left(-\nu-2 \rho_{c}\right): \mathcal{L}_{n}\left(-\mu-2 \rho_{c}\right)\right], \quad \mu, \nu \in X_{m+n} .
$$

Let $\beta=-n\left(\delta_{-m}^{\prime}+\cdots+\delta_{-1}^{\prime}\right)+m\left(\delta_{1}^{\prime}+\cdots+\delta_{n}^{\prime}\right)$ be the sum of all the negative roots of $\mathfrak{g l}(m+n)$ that are not roots of $\mathfrak{g l}(m) \oplus \mathfrak{g l}(n)$. Then Theorem 6.7 (and Lemma 7.4) of [So3] imply the following character formula for the tilting module $\mathcal{U}_{n}(\mu)$ in $\mathcal{O}_{m+n}^{+}$:

$$
\left(\mathcal{U}_{n}(\mu): \mathcal{K}_{n}(\nu)\right)=\left[\mathcal{K}_{n}\left(\beta-w_{0} \nu\right): \mathcal{L}_{n}\left(\beta-w_{0} \mu\right)\right], \quad \mu, \nu \in X_{m+n}^{+} .
$$

The formula is best understood by the following formula:

$$
f_{\beta-w_{0} \mu}=(m-n+1) \mathbf{1}^{m+n}-f_{\mu} \cdot w_{0},
$$

where $\mathbf{1}^{m+n}=(1, \cdots, 1 \mid 1, \cdots, 1) \in \mathbb{Z}^{m+n}$. So the difference of the parameters on both sides of (5.3) is essentially given by the symmetry $f \mapsto-f \cdot w_{0}$ (a similar phenomenon has been observed in (4.17) of [Br1] for $\mathfrak{g l}(m \mid n))$.

5.2. The Jantzen irreducibility criterion. The following is obtained by applying Jantzen's irreducibility criterion $(\mathrm{M}+)$ [Ja1, Satz 4] to $\mathcal{K}_{n}(\lambda)$.

Proposition 5.1. Let $n \in \mathbb{N}$. The $\mathfrak{g l}(m+n)$-module $\mathcal{K}_{n}(\lambda)$ is irreducible if and only if for any $\beta$ of the form $\delta_{i}^{\prime}-\delta_{j}^{\prime}, i<0<j$, with $\left(\lambda+\rho_{c} \mid \beta\right)_{c}$ being a positive integer, there exists an $\alpha$ of the form $\delta_{i}^{\prime}-\delta_{l}^{\prime}, 0<l$, or $\delta_{k}^{\prime}-\delta_{j}^{\prime}, k<0$, with $\left(\lambda+\rho_{c} \mid \alpha\right)_{c}=0$.

Jantzen's criterion can be simplified for $\mathfrak{g l}(m+\infty)$.

Proposition 5.2. The $\mathfrak{g l}(m+\infty)$-module $\mathcal{K}(\lambda)$ is irreducible if and only if for every $-m \leq i<0$, there exists an $\ell>0$ with $\left(\lambda+\rho_{c} \mid \delta_{i}^{\prime}-\delta_{\ell}^{\prime}\right)_{c}=0$.

Proof. As Jantzen's criterion is obtained by the non-degeneracy of the contravariant bilinear form on the generalized Verma module $\mathcal{K}(\lambda)$, it works for $n=\infty$ as well. Let us call the criterion for $n=\infty$ in Proposition 5.1 Condition (A) and the one in Proposition 5.2 Condition (B). Apparently Condition (B) implies (A). 
On the other hand, assume that (A) is satisfied. Let $i$ be such that $-m \leq i<0$. Since $\left(\lambda+\rho_{c} \mid \delta_{k}^{\prime}-\delta_{J+1}^{\prime}\right)_{c}>\left(\lambda+\rho_{c} \mid \delta_{k}^{\prime}-\delta_{J}^{\prime}\right)_{c}$, we can take $J \gg 0$ such that $\left(\lambda+\rho_{c} \mid \delta_{k}^{\prime}-\delta_{J}^{\prime}\right)_{c}>0$ for every $-m \leq k \leq-1$. In particular, for the root $\beta=\delta_{i}^{\prime}-\delta_{J}^{\prime}$ the number $\left(\lambda+\rho_{c} \mid \beta\right)_{c}$ is a positive integer. Applying Condition (A) to $\beta$ now gives us Condition $(\mathrm{B})$.

5.3. Kazhdan-Lusztig polynomials and (dual) canonical bases. In KL the Kazhdan-Lusztig polynomials were introduced and a parabolic version was later defined by Deodhar Deo (also [CC]). Here we follow the presentation in So2.

Throughout this subsection we assume that $f$ is anti-dominant (see Subsection 4.1). Let $S_{f}$ denote the stabilizer subgroup (of $S_{m+n}$ ) of $f$. Denote by $\mathcal{H}_{f}$ the Iwahori-Hecke algebra associated to $S_{f}$. Denote by $\mathbf{1}_{\mathcal{H}_{f}}$ the one-dimensional right $\mathcal{H}_{f}$-module with basis element 1 such that $1 \cdot H_{i}=q^{-1} 1$. Set

$$
\mathcal{M}^{f}=\mathbf{1}_{\mathcal{H}_{f}} \otimes_{\mathcal{H}_{f}} \mathcal{H}_{m+n}, \text { and } M_{x}=1 \otimes H_{x}, \quad x \in S_{m+n} .
$$

$\mathcal{M}^{f}$ carries a natural right $\mathcal{H}_{m+n}$-module structure. Denote by $S^{f}$ the set of minimal length representatives for the right cosets $S_{f} \backslash S_{m+n}$. Then $\mathcal{M}^{f}$ has a basis $M_{x}, x \in S^{f}$. There exists a unique bar involution on $\mathcal{M}^{f}$ which satisfies $\overline{M_{x}}=1 \otimes \overline{H_{x}}$ and $\overline{M H}=\bar{M} \cdot \bar{H}$ for all $M \in \mathcal{M}^{f}, H \in \mathcal{H}_{m+n}$. According to [KL, Deo, So2], $\mathcal{M}^{f}$ admits the Kazhdan-Lusztig bases $\left\{\underline{M}_{x}\right\},\left\{\underline{\tilde{M}}_{x}\right\}$, where $x \in S^{f}$, which are characterized by the following properties:

(a) every $\underline{M}_{x}, \underline{\tilde{M}}_{x}$ with $x \in S^{f}$ is bar-invariant;

(b) $\underline{M}_{x}=M_{x}+\sum_{y} m_{y, x}(q) M_{y}, \underline{\tilde{M}}_{x}=M_{x}+\sum_{y} \tilde{m}_{y, x}(q) M_{y}$, where $m_{y, x} \in q \mathbb{Z}[q]$ and $\tilde{m}_{y, x} \in q^{-1} \mathbb{Z}\left[q^{-1}\right]$. (Furthermore, it is known that $m_{y, x}=0=\tilde{m}_{y, x}$ unless $y<x$. By convention we set $m_{x, x}(q)=\tilde{m}_{x, x}(q)=1$.)

By examining the $\mathcal{H}_{m+n}$-module homomorphism $\phi: \mathcal{M}^{f} \rightarrow \mathbb{T}^{m+n}, M_{x} \mapsto \mathcal{V}_{f \cdot x}$, one has the following well-known identification (cf. [So2, Br1, FKK]).

Proposition 5.3. Suppose that $f \in \mathbb{Z}^{m+n}$ is anti-dominant. Then in $\mathbb{T}^{m+n}$ we have

$$
\mathcal{T}_{f \cdot x}=\mathcal{V}_{f \cdot x}+\sum_{y<x} m_{y, x}(q) \mathcal{V}_{f \cdot y}, \quad \mathcal{L}_{f \cdot x}=\mathcal{V}_{f \cdot x}+\sum_{y<x} \tilde{m}_{y, x}(q) \mathcal{V}_{f \cdot y} .
$$

Equivalently, we have

$$
\begin{aligned}
& \mathfrak{t}_{g, f \cdot x}(q)=m_{y, x}(q), \text { if } g=f \cdot y \text { for some } y \in S^{f} \\
& \mathfrak{l}_{g, f \cdot x}(q)=\tilde{m}_{y, x}(q), \text { if } g=f \cdot y \text { for some } y \in S^{f}
\end{aligned}
$$

and $\mathfrak{t}_{g, f \cdot x}, \mathfrak{l}_{g, f \cdot x}$ are zero otherwise.

5.4. Kazhdan-Lusztig conjecture and (dual) canonical bases. We write $\mathfrak{l}_{g, f}(q), \mathfrak{t}_{g, f}(q)$ for $\mathfrak{l}_{\mu, \lambda}(q), \mathfrak{t}_{\mu, \lambda}(q)$, where $f, g$ correspond to $\lambda, \mu$, respectively, under the bijection $X_{m+n}^{+} \rightarrow \mathbb{Z}_{+}^{m+n}$.

The following theorem is a reformulation in terms of dual canonical and canonical bases of the Kazhdan-Lusztig conjecture proved in BB, BK combined with the translation principle of Jantzen (cf. CC, So1, BGS, Ja2]) and the character formula of tilting modules So3. The proof here is inspired by a similar argument as in the proof of Theorem 4.31 in Br1]. Such a reformulation is known to experts. 
Theorem 5.4. (1) In the Grothendieck group $G\left(\mathcal{O}_{m+n}\right)$, for $\nu \in X_{m+n}$, we have

$$
\left[\mathcal{T}_{n}(\nu)\right]=\sum_{\mu \in X_{m+n}} \mathfrak{t}_{\mu, \nu}(1)\left[\mathcal{V}_{n}(\mu)\right], \quad\left[\mathcal{L}_{n}(\nu)\right]=\sum_{\mu \in X_{m+n}} \mathfrak{l}_{\mu, \nu}(1)\left[\mathcal{V}_{n}(\mu)\right] .
$$

(2) In the Grothendieck group $G\left(\mathcal{O}_{m+n}^{+}\right)$, for $\nu \in X_{m+n}^{+}$, we have

$$
\left[\mathcal{U}_{n}(\nu)\right]=\sum_{\mu \in X_{m+n}^{+}} \mathfrak{u}_{\mu, \nu}(1)\left[\mathcal{K}_{n}(\mu)\right], \quad\left[\mathcal{L}_{n}(\nu)\right]=\sum_{\mu \in X_{m+n}^{+}} \mathfrak{l}_{\mu, \nu}(1)\left[\mathcal{K}_{n}(\mu)\right] .
$$

Proof. Let $\lambda \in X_{m+n}$ be such that $f_{-\lambda-2 \rho_{c}} \in \mathbb{Z}^{m+n}$ satisfies that $f_{-\lambda-2 \rho_{c}}(-m) \leq$ $\cdots \leq f_{-\lambda-2 \rho_{c}}(n)$. The dot action of the Weyl group $S_{m+n}$ on $X_{m+n}$ is given by $\sigma \cdot \mu=\sigma\left(\mu+\rho_{c}\right)-\rho_{c}$, where $\sigma \in S_{m+n}, \mu \in X_{m+n}$. Let $W_{\lambda}$ be the stabilizer (in $\left.S_{m+n}\right)$ of $\lambda$ under the dot action, $W^{\lambda}$ the set of maximal length representatives of the left cosets $S_{m+n} / W_{\lambda}$, and $w_{\lambda}$ the longest element in $W_{\lambda}$. By the KazhdanLusztig conjecture, combined with the translation principle, we have

$$
\left[\mathcal{V}_{n}(\sigma \cdot \lambda): \mathcal{L}_{n}(\tau \cdot \lambda)\right]=P_{\sigma, \tau}(1)=m_{w_{\lambda} \sigma^{-1}, w_{\lambda} \tau^{-1}}(1), \quad \sigma, \tau \in W^{\lambda} .
$$

Here $P_{\sigma, \tau}(1)$ is the value at 1 of the usual Kazhdan-Lusztig polynomial [KL, and it is equal to $m_{w_{\lambda} \sigma^{-1}, w_{\lambda} \tau^{-1}}(1)$ in the notation of the previous subsection, according to [So2]. By Proposition 5.3 we have $m_{w_{\lambda} \sigma^{-1}, w_{\lambda} \tau^{-1}}(1)=\mathfrak{t}_{-\sigma \cdot \lambda-2 \rho_{c},-\tau \cdot \lambda-2 \rho_{c}}(1)$ for $\sigma, \tau \in W^{\lambda}$. Thus by (5.5) we have

$$
\left[\mathcal{V}_{n}(\sigma \cdot \lambda): \mathcal{L}_{n}(\tau \cdot \lambda)\right]=\mathfrak{t}_{-\sigma \cdot \lambda-2 \rho_{c},-\tau \cdot \lambda-2 \rho_{c}}(1) .
$$

Combining (5.2) and (5.6) we have proved the first identity in part (1).

By Remark 4.3 , the matrices $\left[\mathfrak{t}_{-\sigma \cdot \lambda-2 \rho_{c},-\tau \cdot \lambda-2 \rho_{c}}(1)\right]$ and $\left[\mathfrak{l}_{\sigma \cdot \lambda, \tau \cdot \lambda}(1)\right]$ are inverses to each other. The second identity in (1) follows from this.

The second identity in (2) can be derived from the second identity in (1) and the Weyl character formula (applied to $\mathfrak{g l}(m) \oplus \mathfrak{g l}(n))$ (cf. [So3, Sect. 7]). By Remark4.8, the matrices $\left[\mathfrak{u}_{-f_{\mu} \cdot w_{0},-f_{\nu} \cdot w_{0}}(1)\right]$ and $\left[\mathfrak{l}_{f_{\mu}, f_{\nu}}(1)\right]$ are inverses to each other. Now the remaining identity in (2) follows by applying (5.3) and (5.4).

Note that a weight $\lambda \in X_{m+n}^{+}$is $J$-typical (i.e. $\lambda$ is minimal in $X_{m+n}^{+}$in the Bruhat ordering) if and only if the generalized Verma module $\mathcal{K}_{n}(\lambda)$ is irreducible.

5.5. The categories $\mathcal{O}_{m+n}^{++}$and $\mathcal{O}_{m+\infty}^{++}$. In what follows by wt ${ }^{\epsilon}$ we mean the definition given in (2.1). Denote by $\chi_{\lambda}$ the integral central character associated to $\lambda \in X_{m+n}$. It is known that $\chi_{\lambda}=\chi_{\mu}$ for $\lambda, \mu \in X_{m+n}$ if and only if $\lambda=\sigma \cdot \mu$ for some $\sigma \in S_{m+n}$, or equivalently $\mathrm{wt}^{\epsilon}(\lambda)=\mathrm{wt}^{\epsilon}(\mu) \in P$. We denote by $\mathcal{O}_{\gamma}^{+}$the block in $\mathcal{O}_{m+n}^{+}$associated to $\gamma \in P$, denote by $\operatorname{pr}_{\gamma}$ the projection functor from $\mathcal{O}_{m+n}^{+}$to $\mathrm{O}_{\gamma}^{+}$.

Let $V$ be the natural $\mathfrak{g l}(m+n)$-module and $V^{*}$ its dual. For $a \in \mathbb{Z}$ we define the translation functors $E_{a}, F_{a}: \mathcal{O}_{m+n}^{+} \longrightarrow \mathcal{O}_{m+n}^{+}$by sending $M \in \mathcal{O}_{\gamma}^{+}$to

$$
F_{a} M:=\operatorname{pr}_{\gamma-\left(\epsilon_{a}-\epsilon_{a+1}\right)}(M \otimes V), \quad E_{a} M:=\operatorname{pr}_{\gamma+\left(\epsilon_{a}-\epsilon_{a+1}\right)}\left(M \otimes V^{*}\right) .
$$

Let $G\left(\mathcal{O}_{m+n}^{+}\right)_{\mathbb{Q}}:=G\left(\mathcal{O}_{m+n}^{+}\right) \otimes_{\mathbb{Z}} \mathbb{Q}$ and let $\left.\mathcal{E}^{m+n}\right|_{q=1}$ be the specialization of $\mathcal{E}^{m+n}$ at $q \rightarrow 1$.

Theorem 5.5. Let $n \in \mathbb{N}$.

(1) Sending the Chevalley generators $E_{a}, F_{a}$ to the translation functors $E_{a}, F_{a}$ defines a $\mathfrak{U}_{q=1}$-module structure on $G\left(\mathcal{O}_{m+n}^{+}\right) \mathbb{Q}$. 
(2) The linear map $i:\left.G\left(\mathcal{O}_{m+n}^{+}\right)_{\mathbb{Q}} \rightarrow \mathcal{E}^{m+n}\right|_{q=1}$ which sends $\left[\mathcal{K}_{n}(\lambda)\right]$ to $\mathcal{K}_{f_{\lambda}}(1)$ for each $\lambda \in X_{m+n}^{+}$is an isomorphism of $\mathcal{U}_{q=1}$-modules.

(3) The map $i$ sends $\left[\mathcal{U}_{n}(\lambda)\right]$ to $\mathcal{U}_{f_{\lambda}}(1)$ and $\left[\mathcal{L}_{n}(\lambda)\right]$ to $\mathcal{L}_{f_{\lambda}}(1)$, for each $\lambda \in$ $X_{m+n}^{+}$.

Proof. The map $i$ is certainly a vector space isomorphism. One checks that the action of the translation functors on the generalized Verma modules is compatible with the action of the Chevalley generators of $\mathfrak{U}_{q=1}$ on the monomial basis. Thus (1) and (2) follow. Now (3) follows from Theorem 5.4 and the definition of the KL polynomials $\mathfrak{u}_{\mu, \nu}$ and $\mathfrak{l}_{\mu, \nu}$.

Corollary 5.6. Let $\lambda \in X_{m+n}^{+}$. The subquotients of a generalized Verma flag of $\mathcal{U}_{n}(\lambda)$ are precisely $\mathcal{K}_{n}(\mu)$ with $\mu=\mathbb{L}_{\theta}(\lambda)$ associated to $\theta \in\{0,1\} * f_{\lambda}$. Furthermore for $X_{a} \in\left\{E_{a}, F_{a}\right\}$ corresponding to the Chevalley operators in Procedure 4.11, $\mathcal{X}_{a} \mathcal{U}_{n}(\lambda)$ is a tilting module. Also we have $\mathcal{U}_{n}(\lambda)^{\tau} \cong \mathcal{U}_{n}(\lambda)$.

The corollary above can be proved using induction based on Procedure 4.11 in an analogous way as Theorem 4.37 in $\mathrm{Br} 1$ is proved. The induction procedure also shows that the indexing set $\left\{\mu=\mathbb{L}_{\theta}(\lambda) \mid \theta \in\{0,1\} * f_{\lambda}\right\}$ above is compatible with the indexing set in Theorem 4.16 for $f=f_{\lambda}$. Finally $\mathcal{U}_{n}(\lambda)^{\tau} \cong \mathcal{U}_{n}(\lambda)$ is a consequence of Procedure 4.11 and the fact that $\tau$ commutes with the translation functors.

Theorem 5.5 and results from Section 4 imply the stability of the composition factors in a generalized Verma module, and the stability of generalized Verma flags in a tilting module. This allows us to apply the machinery of Section 3 to the reductive setting. We will state these results below.

Given $\lambda, \mu \in X_{m+\infty}^{+}$, we may regard $\lambda, \mu \in X_{m+n}^{++}$for $n \gg 0$. Then,

$$
[\mathcal{K}(\lambda): \mathcal{L}(\mu)]=\left[\mathcal{K}_{n}(\lambda): \mathcal{L}_{n}(\mu)\right], \quad \text { for } n \gg 0 .
$$

For $n<n^{\prime} \leq \infty$, the truncation functor $\operatorname{tr}_{n^{\prime}, n}: \mathcal{O}_{m+n^{\prime}}^{++} \longrightarrow \mathcal{O}_{m+n}^{++}$is defined by sending an object $M$ to the $\mathfrak{g l}(m+n)$-module

$$
\operatorname{tr}_{n^{\prime}, n}(M):=\operatorname{span}\left\{v \in M \mid\left(\operatorname{wt}(v) \mid \delta_{k}^{\prime}\right)_{c}=0 \text { for all } n+1 \leq k \leq n^{\prime}\right\} .
$$

When $n^{\prime}$ is clear from the context we will also write $\operatorname{tr}_{n}$ for $\operatorname{tr}_{n^{\prime}, n}$. Theorem 5.5 allows us to apply the construction of Section 3 to prove the existence of a unique tilting module $\mathcal{U}(\lambda)$ associated to $\lambda$ in $\mathcal{O}_{m \mid \infty}^{++}$and isomorphic to $\bigcup_{n} \mathcal{U}_{n}(\lambda)$. Moreover,

$$
(\mathcal{U}(\lambda): \mathcal{K}(\mu))=\left(\mathcal{U}_{n}(\lambda): \mathcal{K}_{n}(\mu)\right), \quad \text { for } n \gg 0,
$$

and $\mathcal{U}(\lambda)$ has a generalized Verma flag whose subquotients are precisely $\mathcal{K}(\mu)$ with $\mu=\mathbb{L}_{\theta}(\lambda)$ associated to $\theta \in\{0,1\}^{*} f_{\lambda}$. Furthermore, $U(\lambda)^{\tau} \cong U(\lambda)$.

Let $n<n^{\prime} \leq \infty$ and $\lambda \in X_{m+n^{\prime}}^{++}$. Then $\operatorname{tr}_{n}$ sends $y_{n^{\prime}}(\lambda)$ to $y_{n}(\lambda)$ if $\left(\lambda \mid \delta_{n+1}^{\prime}\right)_{c}=0$ and to 0 otherwise, for $y=\mathcal{L}, \mathcal{K}$ or $\mathcal{U}$.

Similarly one proves the reductive analogue of Proposition 3.17 .

Proposition 5.7. For a suitable topological completion $\widehat{G}\left(\mathcal{O}_{m+\infty}^{++}\right) \mathbb{Q}$ of $G\left(\mathcal{O}_{m+\infty}^{++}\right)_{\mathbb{Q}}$, the linear map $i:\left.\widehat{G}\left(\mathcal{O}_{m+\infty}^{++}\right)_{\mathbb{Q}} \rightarrow \widehat{\mathcal{E}}^{m+\infty}\right|_{q=1}$ which sends $[\mathcal{K}(\lambda)]$ to $\mathcal{K}_{f_{\lambda}}(1)$ for each $\lambda \in X_{m+\infty}^{+}$is an isomorphism of vector spaces. The map $i$ further identifies $[\mathcal{U}(\lambda)]$ with $\mathcal{U}_{f_{\lambda}}(1)$ and $[\mathcal{L}(\lambda)]$ with $\mathcal{L}_{f_{\lambda}}(1)$. 


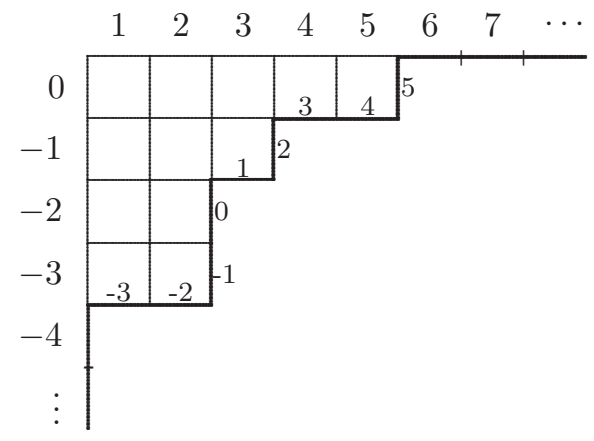

FiguRe 1

\section{Super duality}

\subsection{An isomorphism of Fock spaces.}

Proposition 6.1. (1) The $\mathcal{U}$-module $\Lambda^{\infty} \mathbb{V}$ is isomorphic to the basic representation of $\mathcal{U}$ with highest weight vector $|0\rangle=v_{0} \wedge v_{-1} \wedge v_{-2} \wedge \cdots$. More explicitly, $E_{a}|0\rangle=0$ and $K_{a, a+1}|0\rangle=q^{\delta_{a, 0}}|0\rangle$, for all $a \in \mathbb{Z}$.

(2) The $\mathcal{U}$-module $\Lambda^{\infty} \mathbb{V}^{*}$ is isomorphic to the basic representation of $\mathcal{U}$ with highest weight vector $\left|0_{*}\right\rangle=w_{1} \wedge w_{2} \wedge w_{3} \wedge \cdots$.

Proof. We prove (2). Using the formulas of the $\mathcal{U}$-action on $\mathbb{W}$, we verify that:

a) $E_{a}\left|0_{*}\right\rangle=0$ for every $a \in \mathbb{Z}$;

b) $K_{a}\left|0_{*}\right\rangle=\left|0_{*}\right\rangle, a \leq 0$ and $K_{b}\left|0_{*}\right\rangle=q^{-1}\left|0_{*}\right\rangle, b>0$.

This implies that the weights of $\left|0_{*}\right\rangle$ by $K_{a, a+1}=K_{a} K_{a+1}^{-1}\left(=q^{h_{a}}\right)$ are 1 for $a \neq 0$ and $q$ for $a=0$. This says that the weight is the fundamental weight $\Lambda_{0}$. The claim now follows from the fact that $\Lambda^{\infty} \mathbb{V}^{*}$ and the basic representation of $\mathcal{U}$ have the same character.

Part (1) can be proved similarly and can be found in MM, KMS.

Thus we have obtained a natural isomorphism of $\mathcal{U}$-modules

$$
C: \Lambda^{\infty} \mathbb{V} \stackrel{\cong}{\cong} \Lambda^{\infty} \mathbb{V}^{*} \text {. }
$$

Consider the lattice on the fourth quadrant of the $(x, y)$-plane. Let us label unit intervals on the $x$-axis $1,2,3, \cdots$ and those on the $y$-axis $0,-1,-2, \cdots$. (See Figure 1.) A lattice path has one end going down vertically along the $y$-axis and the other end going to the right horizontally along the $x$-axis. Clearly, there is a bijective correspondence between such lattice paths and Young diagrams.

The edges of a lattice path are further labeled by integers, and the labels are uniquely determined by two requirements: a) the set of all labels coincides with $\mathbb{Z}$; b) the labels on the edges which lie on the $x$ - and $y$-axes coincide with the pre-fixed labels therein. By abuse of notation, we will use $\lambda$ to refer to both a partition and its associated lattice path. Note that our labeling differs from the one in MM, p. 81 , by a shift. Denote by $\operatorname{vL}(\lambda)$ the set of vertical labels of $\lambda$ and by $\operatorname{hL}(\lambda)$ the set of horizontal labels of $\lambda$. For example, associated to the partition $\lambda=(5,3,2,2)$, the lattice path is the bold line segments with labels attached.

The following lemma seems to be well known. 
Lemma 6.2. Let $\lambda=\left(\lambda_{1}, \lambda_{2}, \cdots\right)$ be a partition, and let $\lambda^{\prime}=\left(\lambda_{1}^{\prime}, \lambda_{2}^{\prime}, \cdots\right)$ be its conjugate partition. Then,

(1) the set of horizontal labels $h L(\lambda)$ is $\left\{i-\lambda_{i}^{\prime}, i=1,2, \cdots\right\}$;

(2) the set of vertical labels $v L(\lambda)$ is $\left\{\lambda_{i}-i+1, i=1,2, \cdots\right\}$.

The following observation plays an important role in this paper.

Theorem 6.3. The U-module isomorphism $C: \Lambda^{\infty} \mathbb{V} \rightarrow \Lambda^{\infty} \mathbb{V}^{*}$ is explicitly given by sending $|\lambda\rangle$ to $\left|\lambda_{*}^{\prime}\right\rangle$ for each partition $\lambda$.

Proof. Define the notions of concave and convex corners in a lattice path as follows:

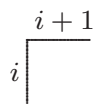

Concave corner

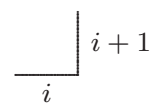

Convex corner

Figure 2

With our convention of labeling, the formulas in $\mathrm{MM}$, pp. 81-82, can be adjusted as follows, e.g. for $F_{i}$. We have $F_{i}|\lambda\rangle=|\nu\rangle$ if $\lambda$ has the concave corner labeled by $i, i+1$ (see Figure 2) (instead of the $i-1, i$ in [MM]) and $\nu$ is the same as $\lambda$ except this corner becomes convex, labeled again by $i, i+1$, i.e., with one particular cell added (see Figure 2). Otherwise $F_{i}|\lambda\rangle$ equals 0 . In other words, the vertical labels of the lattice path of $\lambda$, after changing the label $i$ to $i+1$ (when allowed), become the vertical labels of the lattice path of $\nu$. By Lemma 6.2, the above formula is equivalent to the statement that $F_{i}|\lambda\rangle$ is the semi-infinite wedge obtained by replacing the $v_{i}$ appearing in $|\lambda\rangle$ by $v_{i+1}$. By the way, this is consistent with the action of $\mathcal{U}$ on $\mathbb{V}$.

Now we work out explicitly the action of $F_{i}$ on the basis element $\left|\lambda_{*}^{\prime}\right\rangle$ of $\Lambda^{\infty} \mathbb{V}^{*}$. By the action of $\mathcal{U}$ on $\mathbb{W}, F_{i}\left|\lambda_{*}^{\prime}\right\rangle$ is the semi-infinite wedge in $\Lambda^{\infty} \mathbb{V}^{*}$ which is obtained by replacing the $w_{i+1}$ appearing in $\left|\lambda_{*}^{\prime}\right\rangle$ by $w_{i}$. In light of Lemma 6.2, the horizontal labels of the lattice path associated to $F_{i}\left|\lambda_{*}^{\prime}\right\rangle$ are obtained from those for $\lambda$ by changing the label $i+1$ to $i$ (when allowed). This is exactly the $\nu$ above. Thus we conclude that $F_{i}\left|\lambda_{*}^{\prime}\right\rangle=\left|\nu_{*}^{\prime}\right\rangle$ if $\lambda$ has the concave corner labeled by $i, i+1$; otherwise it is 0 .

One checks similarly that sending $|\lambda\rangle$ to $\left|\lambda_{*}^{\prime}\right\rangle$ respects the actions of $E_{i}, K_{i, i+1}$ for each $i \in \mathbb{Z}$.

6.2. The match of typicality and $J$-typicality. Given $\lambda=\sum_{i \in I(m \mid \infty)} \lambda_{i} \delta_{i}^{\prime} \in$ $X_{m+\infty}^{+}$so that by definition $\lambda^{>0}:=\left(\lambda_{1}, \lambda_{2}, \cdots\right)$ is a partition, and denoting by $\left(\lambda_{1}^{\prime}, \lambda_{2}^{\prime}, \cdots\right)$ the conjugate partition of $\lambda^{>0}$, we define a weight in $X_{m \mid \infty}^{+}$by

$$
\lambda^{\natural}:=\sum_{i=-m}^{-1} \lambda_{i} \delta_{i}+\sum_{j=1}^{\infty} \lambda_{j}^{\prime} \delta_{j} .
$$

This actually defines a bijection $X_{m+\infty}^{+} \stackrel{\natural}{\longleftrightarrow} X_{m \mid \infty}^{+}$. 
Theorem 6.4. (1) For every $\lambda \in X_{m+\infty}^{+}$, the degree of $J$-atypicality of the weight $\lambda \in X_{m+\infty}^{+}$is equal to the degree of atypicality of $\lambda^{\natural}$.

(2) A weight $\lambda \in X_{m+\infty}^{+}$is J-typical if and only if $\lambda^{\natural} \in X_{m \mid \infty}^{+}$is typical.

Proof. Part (2) is a special case of (1), and thus it suffices to prove (1).

Let us write $\lambda=\left(\lambda^{<0} \mid \lambda^{>0}\right)$ as usual. For the proof it will be convenient to put $\mu=\lambda^{>0}$. By definition, the degree of atypicality of $\lambda^{\natural}=\left(\lambda^{<0} \mid \mu^{\prime}\right)$ is the number of $i$ 's in $\{-m, \cdots,-1\}$ such that

$$
\left(\lambda^{\natural}+\rho \mid \delta_{i}-\delta_{j}\right)=\lambda_{i}-i+\mu_{j}^{\prime}-j=0, \quad \text { for some } j>0,
$$

or by Lemma 6.2. it is the number of $i$ 's in $\{-m, \cdots,-1\}$ such that $\lambda_{i}-i$ is a horizontal label of the lattice path $\mu$.

By definition, the degree of $J$-atypicality of $\lambda=\left(\lambda^{<0} \mid \mu\right)$ is the number of $i$ 's in $\{-m, \cdots,-1\}$ such that

$$
\left(\lambda+\rho_{c} \mid \delta_{i}^{\prime}-\delta_{\ell}^{\prime}\right)_{c}=\lambda_{i}-i-\mu_{\ell}+\ell-1 \neq 0, \text { for every } \ell>0,
$$

or by Lemma 6.2 it is the number of $i$ 's in $\{-m, \cdots,-1\}$ such that $\lambda_{i}-i$ is not a vertical label of the lattice path $\mu$.

Now the theorem follows because the set of horizontal labels of $\mu$ and the set of vertical labels of $\mu$ are disjoint and their union is $\mathbb{Z}$.

Remark 6.5. From the above proof, we observe that the subset of $\{-m, \cdots,-1\}$ that contributes to the $J$-atypicality of $\lambda$ coincides with the one that contributes to the atypicality of $\lambda^{\natural}$.

6.3. The canonical isomorphism. By coupling with the two bijections $X_{m \mid \infty}^{+} \rightarrow$ $\mathbb{Z}_{+}^{m \mid \infty}$ and $X_{m+\infty}^{+} \rightarrow \mathbb{Z}_{+}^{m+\infty}$, the bijection $X_{m+\infty}^{+} \stackrel{\natural}{\longleftrightarrow} X_{m \mid \infty}^{+}$induces a bijection $\mathbb{Z}_{+}^{m+\infty} \stackrel{\natural}{\longleftrightarrow} \mathbb{Z}_{+}^{m \mid \infty}$, which will also be denoted by $\natural$.

Lemma 6.6. For $f, g \in \mathbb{Z}_{+}^{m+\infty}, f \geq g$ in the Bruhat ordering if and only if $f^{\natural} \succcurlyeq g^{\natural}$ in the super Bruhat ordering.

Proof. First we note that $h \succ e$ in the super Bruhat ordering for $e, h \in \mathbb{Z}_{+}^{m \mid \infty}$ if and only if there exists a sequence $h_{0}, h_{1}, \ldots, h_{t} \in \mathbb{Z}_{+}^{m \mid \infty}$ such that $h=h_{0} \succ h_{1} \succ$ $\cdots \succ h_{t}=e$ and for each $0 \leq a<t, h_{a+1}=\left(h_{a}-r\left(d_{i_{a}}-d_{j_{a}}\right)\right)^{+}$for some $r>0$ and $i_{a}<0<j_{a}$ with $h_{a}\left(i_{a}\right)=h_{a}\left(j_{a}\right)$. This claim can be deduced from the proof of Lemma 3.42 in $\mathrm{Br} 1$ using the equivalent formulation of the super Bruhat ordering in terms of $\mathrm{wt}^{\epsilon}$ and some simple inequalities (cf. $\S 2$-b, (2.4) in [Br1]). We shall say that $h_{a}$ is reduced to $h_{a+1}$ by a super move. Denote by $\left(\lambda_{<}^{a} \mid \lambda^{a}\right) \in X_{m \mid \infty}^{+}$the weight corresponding to $h_{a}$. The horizontal label set $\mathrm{hL}\left(\lambda^{a+1}\right)$ is obtained from $\mathrm{hL}\left(\lambda^{a}\right)$ by substituting $h_{a}\left(j_{a}\right)$ with the smaller number $h_{a}\left(j_{a}\right)-r$, and this statement in turn characterizes a super move.

On the other hand, recall from Lemma 4.4 that $f>g$ in the Bruhat ordering if and only if there exists $f^{0}, \cdots, f^{s} \in \mathbb{Z}_{+}^{m+\infty}$ such that $f=f^{0}>f^{1}>\cdots>f^{s}=g$ and for each $0 \leq a<s$ there exists $i_{a}<0<j_{a}$ with $f^{a+1}=\left(f^{a} \cdot \tau_{i_{a} j_{a}}\right)^{+}, f^{a}\left(i_{a}\right)>$ $f^{a}\left(j_{a}\right)$, and $f^{a}\left(i_{a}\right) \neq f^{a}(k), \forall k>0$. We shall say $f^{a}$ is reduced to $f^{a+1}$ by a simple move. Note that $f^{a}\left(i_{a}\right)$ does not belong to the vertical label set $\operatorname{vL}\left(\mu^{a}\right)$ if we denote by $\left(\mu_{<}^{a} \mid \mu^{a}\right) \in X_{m+\infty}^{+}$the weight corresponding to $f^{a}$. The set $\operatorname{vL}\left(\mu^{a+1}\right)$ is obtained from $\operatorname{vL}\left(\mu^{a}\right)$ by substituting $f^{a}\left(j_{a}\right)$ with the larger number $f^{a}\left(i_{a}\right)$, and this statement in turn characterizes a simple move. By Remark 6.5, Lemma 6.2. 
and the fact that $\mathbb{Z}=\mathrm{hL}(\nu) \sqcup \mathrm{vL}(\nu)$ for any partition $\nu$, we conclude that if $f$ is reduced to $g$ by a simple move, then $f^{\natural}$ is reduced to $g^{\natural}$ by a super move, and vice versa. An induction on the number of simple/super moves completes the proof.

The U-module isomorphism $C: \Lambda^{\infty} \mathbb{V} \rightarrow \Lambda^{\infty} \mathbb{V}^{*}$ gives rise to an isomorphism of $\mathcal{U}$-modules $\mathcal{E}^{m+\infty} \stackrel{\cong}{\cong} \mathcal{E}^{m \mid \infty}$. Recall that $\widehat{\mathcal{E}}^{m+\infty}$ and $\widehat{\mathcal{E}}^{m \mid \infty}$ are topological completions of $\Lambda^{m} \mathbb{V} \otimes \Lambda^{\infty} \mathbb{V}$ and $\Lambda^{m} \mathbb{V} \otimes \Lambda^{\infty} \mathbb{V}^{*}$, respectively. Using the fact that $\mathbb{Z}=\mathrm{hL}(\lambda) \sqcup \mathrm{vL}(\lambda)$ for a partition $\lambda$, one can show that these two completions are indeed compatible under the above map. Hence we obtain a natural isomorphism of $\mathcal{U}$-modules (which will also be conveniently denoted by $\sharp$ by abuse of notation):

$$
\natural=1 \otimes C: \widehat{\mathcal{E}}^{m+\infty} \stackrel{\cong}{\longrightarrow} \widehat{\mathcal{E}}^{m \mid \infty} .
$$

Theorem 6.7. The isomorphism $\natural: \widehat{\mathcal{E}}^{m+\infty} \longrightarrow \widehat{\mathcal{E}}^{m \mid \infty}$ has the following properties:

(1) $\natural\left(\mathcal{K}_{f}\right)=K_{f \natural}$ for each $f \in \mathbb{Z}_{+}^{m+\infty}$;

(2) $\natural$ is compatible with the bar involutions, i.e., $\boxminus(\bar{u})=\overline{\natural(u)}$ for each $u \in \widehat{\mathcal{E}}^{m+\infty}$;

(3) $\natural\left(\mathcal{L}_{f}\right)=L_{f}$ for each $f \in \mathbb{Z}_{+}^{m+\infty}$;

(4) $\downarrow\left(\mathcal{U}_{f}\right)=U_{f \natural}$ for each $f \in \mathbb{Z}_{+}^{m+\infty}$.

Proof. (1) follows from the definitions and Theorem 6.3 (2) follows from Theorems 6.3 and 6.4. Lemma 6.6 and the characterizations of the bar involutions. (3) and (4) follow from (1), (2), Lemma 6.6 and the characterizations of these bases.

Combining the isomorphisms $j, i, \natural$ of Proposition 3.17 Proposition 5.7. Theorem 6.7 respectively, we conclude the following. Denote $\sharp:=j^{-1} \circ$ ६ $\circ$.

Theorem 6.8. (1) There exists a linear isomorphism of Grothendieck groups $\sharp: \widehat{G}\left(\mathcal{O}_{m+\infty}^{++}\right)_{\mathbb{Q}} \rightarrow \widehat{G}\left(\mathcal{O}_{m \mid \infty}^{++}\right)_{\mathbb{Q}}$, which sends $[\mathcal{K}(\lambda)],[\mathcal{U}(\lambda)]$ and $[\mathcal{L}(\lambda)]$ to $\left[K\left(\lambda^{\natural}\right)\right],\left[U\left(\lambda^{\natural}\right)\right]$ and $\left[L\left(\lambda^{\natural}\right)\right]$, respectively, where $\lambda \in X_{m+\infty}^{+}$.

(2) For $\lambda, \mu \in X_{m+\infty}^{+}$, we have

$$
\mathfrak{u}_{\mu, \lambda}(q)=u_{\mu^{\natural}, \lambda^{\natural}}(q), \quad \mathfrak{l}_{\mu, \lambda}(q)=\ell_{\mu^{\natural}, \lambda^{\natural}}(q) .
$$

Remark 6.9. From Vogan's interpretation of the Kazhdan-Lusztig conjecture in terms of the $\mathfrak{u}$-cohomology groups ( $\mathrm{Vo}$, , Conjecture 3.4), Brundan-Serganova's description of the polynomials $\ell_{\mu, \lambda}(q)$ [Se1, Br1, Zou, together with Kostant's ucohomology formula for finite-dimensional irreducible modules of $\mathfrak{g l}(N)$, the computation of the cohomology groups $H^{i}\left(\mathfrak{g l}(m \mid n)_{+1} ; L_{n}\left(\lambda^{\natural}\right)\right)$ in [CZ] can be shown to be equivalent to the identity $\mathfrak{l}_{\mu, \lambda}(q)=\ell_{\mu^{\natural}, \lambda^{\natural}}(q)$ when $\lambda$ and $\mu$ are partitions. In the approach of [CZ] the unitarity of the module $L_{n}\left(\lambda^{\natural}\right)$ when $\lambda$ is a partition is used in a crucial way. As noted in loc. cit., the Weyl character formula for $\mathcal{L}(\lambda)$ translates into a Weyl-type character formula for the $\mathfrak{g l}(m \mid n)$-module $L_{n}\left(\lambda^{\natural}\right)$.

Theorem 6.8 strongly suggests the following.

Conjecture 6.10. The categories $\mathcal{O}_{m \mid \infty}^{++}$and $\mathcal{O}_{m+\infty}^{++}$are equivalent.

Remark 6.11. We summarize the precise relations between the Kazhdan-Lusztig polynomials for $\mathfrak{g l}(m \mid n)$ and the usual Kazhdan-Lusztig polynomials for $\mathfrak{g l}(m+N)$ for finite $n$ and $N$. To compute $u_{\mu, \lambda}(q)$ and $\ell_{\mu, \lambda}(q)$ for fixed $\lambda, \mu \in X_{m \mid n}^{+}$, we may assume by Remark 2.15 that $\lambda, \mu \in X_{m \mid n}^{++}$. Denote by $\lambda_{\infty} \in X_{m \mid \infty}^{+}$the extension of $\lambda$ by zeros, and we have $\lambda_{\infty}^{\natural} \in X_{m+\infty}^{+}$. Write $\lambda_{\infty}^{\natural}=\left(\left(\lambda_{\infty}^{\natural}\right)^{<0} \mid\left(\lambda_{\infty}^{\natural}\right)^{>0}\right)$. Assuming 
the lengths of the partitions $\left(\mu_{\infty}^{\natural}\right)^{>0}$ and $\left(\lambda_{\infty}^{\natural}\right)^{>0}$ are no larger than $N$, we have $\lambda_{\infty}^{\natural,(N)}, \mu_{\infty}^{\natural,(N)} \in X_{m+N}^{++}$. Then,

$$
\begin{aligned}
u_{\mu, \lambda}(q) & =u_{\mu_{\infty}, \lambda_{\infty}}(q) \quad \text { by Theorem } 2.14 \\
& =\mathfrak{u}_{\mu_{\infty}^{\natural}, \lambda_{\infty}^{\natural}}(q) \quad \text { by Theorem 6.8, } \\
& =\mathfrak{u}_{\mu_{\infty}^{\natural,(N)}, \lambda_{\infty}^{\natural,(N)}}(q) \quad \text { by Remark 4.31. }
\end{aligned}
$$

Similarly, we have

$$
\ell_{\mu, \lambda}(q)=\mathfrak{l}_{\mu_{\infty}^{\natural,(N)}, \lambda_{\infty}^{\natural,(N)}}(q) .
$$

Observe that, depending on $\lambda$ and $\mu$, the integer $N$ might be arbitrarily large (respectively small) even if $n$ is small (respectively large). To capture all the KazhdanLusztig polynomials for $\mathfrak{g l}(m \mid n)$ with a fixed $n>0$, we use the Kazhdan-Lusztig polynomials for $\mathfrak{g l}(m+N)$ with arbitrarily large $N$.

One can also reverse the roles of Kazhdan-Lusztig polynomials for Lie algebras and Lie superalgebras above.

\subsection{Examples.}

Example 6.12. Consider the element in $\mathbb{Z}_{+}^{4 \mid \infty}$ given by

$$
f=(0,-1,-3,-4 \mid-2,-1,0,4,5,6,7, \cdots) .
$$

Then $\# f=2$ and we have

$$
\begin{aligned}
& \left(\mathrm{L}_{-4,3}(f)\right)^{+}=(-1,-3,-4,-6 \mid-6,-2,-1,4,5,6, \cdots), \\
& \left(\mathrm{L}_{-3,2}(f)\right)^{+}=(0,-3,-4,-5 \mid-5,-2,0,4,5,6, \cdots), \\
& \left(\mathrm{L}_{-3,2} \circ \mathrm{L}_{-4,3}(f)\right)^{+}=(-3,-4,-5,-6 \mid-6,-5,-2,4,5,6, \cdots) .
\end{aligned}
$$

Now consider

$$
f^{(3)}=(0,-1,-3,-4 \mid-2,-1,0) \in \mathbb{Z}_{+}^{4 \mid 3}
$$

with $\# f^{(3)}=2$. This is Example 3.22 in Br1 in our notational convention. Following Procedure 3.20 in Br1] one obtains the following formula for $U_{f^{(3)}}$ :

$$
\begin{aligned}
U_{(0,-1,-3,-4 \mid-2,-1,0)} & \\
= & F_{-5} F_{-4} E_{-3} F_{-2} F_{-6} F_{-5} E_{-4} F_{-3} E_{-2} F_{-1} K_{(-1,-3,-5,-6 \mid-4,-2,0)} \\
= & K_{(0,-1,-3,-4 \mid-2,-1,0)}+q K_{(-1,-3,-4,-6 \mid-6,-2,-1)} \\
& +q K_{(0,-3,-4,-5 \mid-5,-2,0)}+q^{2} K_{(-3,-4,-5,-6 \mid-6,-5,-2)} .
\end{aligned}
$$

One sees that $U_{f}$ is just $U_{f(3)}$ with tails added everywhere. Under $\downarrow$ we have

$$
f^{\natural}=(0,-1,-3,-4 \mid 3,2,1,-3,-4,-5,-6, \cdots) .
$$

The truncation map from $\mathbb{Z}_{+}^{4+\infty} \rightarrow \mathbb{Z}_{+}^{4+3}$ takes $f^{\natural}$ to

$$
f^{\natural(3)}=(0,-1,-3,-4 \mid 3,2,1) .
$$

Note that $f^{\natural(3)}$ is $J$-typical, and hence

$$
\mathcal{U}_{(0,-1,-3,-4 \mid 3,2,1)}=\mathcal{K}_{(0,-1,-3,-4 \mid 3,2,1)} \cdot
$$

This certainly does not correspond to $U_{(0,-1,-3,-4 \mid-2,-1,0)}$ under $\downarrow$. So the canonical bases of $\widehat{\mathcal{E}}^{m \mid n}$ and $\mathcal{E}^{m+n}$ do not correspond for finite $n$ in general. 
Now consider the $n=\infty$ case. Apply Procedure 4.11 to $f^{\natural}$ repeatedly until we get a $J$-typical element. A straightforward calculation shows that

$$
\begin{aligned}
\mathcal{U}_{f \natural}= & F_{-2} E_{-3} E_{-4} F_{-5} F_{-1} E_{-2} E_{-3} F_{-4} E_{-5} F_{-6} \mathcal{K}_{(-1,-2,-4,-6 \mid 3,2,1-1,-2,-4,-6,-7, \cdots)} \\
= & \mathcal{K}_{(0,-1,-3,-4 \mid 3,2,1,-3,-4,-5,-6, \cdots)}+q \mathcal{K}_{(-1,-3,-4,-6 \mid 3,2,1,0,-3,-4,-5,-7, \cdots)} \\
& +q \mathcal{K}_{(0,-3,-4,-5 \mid 3,2,1,-1,-3,-4,-6,-7, \cdots)} \\
& +q^{2} \mathcal{K}_{(-3,-4,-5,-6 \mid 3,2,1,0,-1,-3,-4,-7,-8 \cdots)} .
\end{aligned}
$$

We have

$$
\begin{aligned}
\left(\mathrm{L}_{-4,3}(f)\right)^{+\natural} & =(-1,-3,-4,-6 \mid 3,2,1,0,-3,-4,-5,-7, \cdots) \\
\left(\mathrm{L}_{-3,2}(f)\right)^{+\natural} & =(0,-3,-4,-5 \mid 3,2,1,-1,-3,-4,-6,-7, \cdots) \\
& =\left(\mathbb{L}_{-3}\left(f^{\natural}\right)\right)^{+}, \\
\left(\mathrm{L}_{-3,2} \circ \mathrm{L}_{-4,3}(f)\right)^{+\natural} & =(-3,-4,-5,-6 \mid 3,2,1,0,-1,-3,-4,-7, \cdots) \\
& =\left(\mathbb{L}_{-3} \circ \mathbb{L}_{-4}\left(f^{\natural}\right)\right)^{+} .
\end{aligned}
$$

Therefore $U_{f}$ corresponds to $\mathcal{U}_{f}$ under the map $\sharp$.

Remark 6.13. From Example 6.12 we see that in the Procedure 3.20 in Br1 and Procedure 4.11 the functions do not correspond at each step under the map $\downarrow$, because the Chevalley generators in general are different at each step. Indeed even in the final reduction the typical function and the $J$-typical function do not correspond under $\sharp$. So the two procedures are different.

Example 6.14. Consider the elements $f=(0,-2 \mid-2,0,3,4,5, \cdots)$ and $g=$ $(-2,-4 \mid-4,-2,3,4,5, \cdots)$ in $\mathbb{Z}^{2 \mid \infty}$. Let us compute $\ell_{g, f}$. The case of $\ell_{g^{(2)}, f^{(2)}}$ is a special case of Example 3.40 in [Br1]. One sees that only for $\theta=(2,2)$ and $\theta=(0,2)$ is it possible to have $\mathrm{R}_{\theta}^{\prime}(g)=f$; i.e., we have

$$
\left(\mathrm{R}_{-1,1}^{2}(g)\right)^{+}=f, \quad\left(\mathrm{R}_{-1,1}^{2} \circ \mathrm{R}_{-2,2}^{2}(g)\right)^{+}=f,
$$

so that $\ell_{g, f}\left(-q^{-1}\right)=q^{2}+q^{4}$.

We have

$f^{\natural}=(0,-2 \mid 2,1,-1,-3,-4,-5,-6, \cdots), g^{\natural}=(-2,-4 \mid 2,1,0,-1,-3,-5,-6, \cdots)$.

It is straightforward to verify that we have

$$
\left(\mathbb{R}_{-1}^{2}\left(g^{\natural}\right)\right)^{+}=f^{\natural}, \quad\left(\mathbb{R}_{-1}^{2} \circ \mathbb{R}_{-2}^{2}\left(g^{\natural}\right)\right)^{+}=f^{\natural},
$$

and furthermore these are the only $\theta$ 's for which $\mathbb{R}_{\theta}^{\prime}\left(g^{\natural}\right)=f^{\natural}$. Thus we have $\mathfrak{l}_{g^{\natural}, f^{\natural}}\left(-q^{-1}\right)=q^{2}+q^{4}$. So $\ell_{g, f}=\mathfrak{l}_{g^{\natural}, f^{\natural}}$.

On the other hand, $f^{\natural(2)}$ is $J$-typical; thus $\mathcal{L}_{f \natural(2)}=\mathcal{K}_{f^{\natural}(2)}$. So we do not have a correspondence between the dual canonical basis elements $L_{f^{(2)}}$ and $\mathcal{L}_{f \natural(2)}$ under $\natural$.

6.5. Application to combinatorial characters and tensor products. Denote by $\omega_{+}$the involution of the ring of symmetric functions in the variables $x_{1}, x_{2}, \cdots$, which in terms of generating series in the indeterminate $t$ is given by

$$
\omega_{+}\left(\prod_{i>0}\left(1-t x_{i}\right)^{-1}\right)=\prod_{i>0}\left(1+t x_{i}\right) .
$$

For a partition $\lambda$ we let $s_{\lambda}\left(x_{1}, x_{2}, \cdots\right)$ denote the Schur function in the variables $\mathbf{x}_{+}:=\left\{x_{1}, x_{2}, \cdots\right\}$. The $s_{\nu}\left(x_{-m}, \cdots, x_{-1}\right)$ for finitely many variables $\mathbf{x}_{-}:=$ 
$\left\{x_{-m}, \cdots, x_{-1}\right\}$ makes sense as a Laurent polynomial for a generalized partition $\nu=\left(\nu_{-m}, \cdots, \nu_{-1}\right)$ which by definition satisfies $\nu_{-m} \geq \cdots \geq \nu_{-1}$ and $\nu_{i} \in \mathbb{Z}$.

Let $\mu=\left(\mu^{<0} \mid \mu^{>0}\right) \in X_{m \mid \infty}^{+}$. Setting $e^{\delta_{i}}=x_{i}$, the character of $K(\mu)$ as a power series in $\mathbb{Z}\left[\left[\mathbf{x}_{-}{ }^{ \pm 1}, \mathbf{x}_{+}\right]\right]$is

$$
\operatorname{ch}_{K(\mu)}=s_{\mu}<0\left(\mathbf{x}_{-}\right) s_{\mu}>0\left(\mathbf{x}_{+}\right) \prod_{i<0<j}\left(1+x_{i}^{-1} x_{j}\right) .
$$

Hence by Proposition 3.17 the character of $L(\lambda)$, where $\lambda \in X_{m \mid \infty}^{+}$, is

$$
\operatorname{ch}_{L(\lambda)}=\sum_{\mu} \ell_{\mu, \lambda}(1) s_{\mu}<0\left(\mathbf{x}_{-}\right) s_{\mu}>0\left(\mathbf{x}_{+}\right) \prod_{-m \leq i<0<j}\left(1+x_{i}^{-1} x_{j}\right) .
$$

Similarly, setting $e^{\delta_{i}^{\prime}}=x_{i}$ and using Proposition 5.7 the characters of $\mathcal{K}(\mu)$ and $\mathcal{L}(\lambda)$, where $\lambda, \mu \in X_{m+\infty}^{+}$, are

$$
\begin{aligned}
\operatorname{ch}_{\mathcal{K}(\mu)} & =s_{\mu}<0\left(\mathbf{x}_{-}\right) s_{\mu}>0\left(\mathbf{x}_{+}\right) \prod_{-m \leq i<0<j}\left(1-x_{i}^{-1} x_{j}\right)^{-1}, \\
\operatorname{ch}_{\mathcal{L}(\lambda)} & =\sum_{\mu} \mathfrak{l}_{\mu, \lambda}(1) s_{\mu}<0\left(\mathbf{x}_{-}\right) s_{\mu}>0\left(\mathbf{x}_{+}\right) \prod_{-m \leq i<0<j}\left(1-x_{i}^{-1} x_{j}\right)^{-1} .
\end{aligned}
$$

Comparing (6.1) and (6.2), we have by Theorem 6.8 (2) for $\lambda \in X_{m \mid \infty}^{+}$and $\mu \in$ $X_{m+\infty}^{+}$

$$
\operatorname{ch}_{L(\lambda)}=\omega_{+}\left(\operatorname{ch}_{\mathcal{L}\left(\lambda^{\natural}\right)}\right), \quad \operatorname{ch}_{\mathcal{L}(\mu)}=\omega_{+}\left(\operatorname{ch}_{L\left(\mu^{\natural}\right)}\right) .
$$

Let $n$ be finite. Let $\lambda \in X_{m \mid n}^{++}$and regard $\lambda \in X_{m \mid \infty}^{+}$. By Corollary 3.6 we have

$$
\operatorname{ch}_{L_{n}(\lambda)}\left(x_{-m}, \cdots, x_{-1}, x_{1}, \cdots, x_{n}\right)=\operatorname{ch}_{L(\lambda)}\left(x_{-m}, \cdots, x_{-1}, x_{1}, \cdots, x_{n}, 0,0, \cdots\right)
$$

by setting the variables $x_{n+1}, x_{n+2}, \cdots$ in $\operatorname{ch}_{L(\lambda)}$ to 0 . (A similar remark applies to $\operatorname{ch}_{\mathcal{L}_{n}(\mu)}$ for $\mu \in X_{m+n}^{++}$.) This together with (6.3) implies the following.

Corollary 6.15. For $\lambda \in X_{m \mid n}^{++}$and $\mu \in X_{m+n}^{++}$, we have

(1) $\operatorname{ch}_{L_{n}(\lambda)}\left(x_{-m}, \cdots, x_{n}\right)=\omega_{+}\left(\operatorname{ch}_{\mathcal{L}\left(\lambda^{\natural}\right)}\right)\left(x_{-m}, \cdots, x_{n}, 0,0, \cdots\right)$;

(2) $\operatorname{ch}_{\mathcal{L}_{n}(\mu)}\left(x_{-m}, \cdots, x_{n}\right)=\omega_{+}\left(\operatorname{ch}_{L\left(\mu^{\natural}\right)}\right)\left(x_{-m}, \cdots, x_{n}, 0,0, \cdots\right)$.

Remark 6.16. When $\lambda^{\natural}$ is a partition, $\operatorname{ch}_{\mathcal{L}\left(\lambda^{\natural}\right)}$ is the Schur function $s_{\lambda^{\natural}}$, and thus, $\omega_{+}\left(s_{\lambda^{\natural}}\right)$ is the hook Schur function associated to the partition $\lambda^{\natural}$ (cf. BR. B . Corollary 6.15 (1) recovers the character formula for the irreducible representation of $\mathfrak{g l}(m \mid n)$ appearing in the tensor powers of the natural module $\mathbb{C}^{m \mid n}[\mathrm{~Sv}, \mathrm{BR}$.

6.6. Isomorphism of Grothendieck rings. We shall define product structures in the completed Grothendieck groups $\widehat{G}\left(\mathcal{O}_{m+\infty}^{++}\right) \mathbb{Q}$ and $\widehat{G}\left(\mathcal{O}_{m \mid \infty}^{++}\right)_{\mathbb{Q}}$ induced by the tensor products of modules in the respective categories. For $\lambda, \mu \in X_{m \mid \infty}^{+}$consider the tensor product of two Kac modules $K(\lambda)$ and $K(\mu)$. In order to compute their product we need to determine the Kac modules appearing in the Kac flag of $K(\lambda) \otimes K(\mu)$. We have

$$
\begin{aligned}
K(\lambda) \otimes K(\mu) & =\left(U(\mathfrak{g l}(m \mid \infty)) \otimes_{U(\mathfrak{p})} L^{0}(\lambda)\right) \otimes\left(U(\mathfrak{g l}(m \mid \infty)) \otimes_{U(\mathfrak{p})} L^{0}(\mu)\right) \\
& \cong U(\mathfrak{g l}(m \mid \infty)) \otimes_{U(\mathfrak{p})}\left(L^{0}(\lambda) \otimes U\left(\mathfrak{g l}(m \mid \infty)_{-1}\right) \otimes L^{0}(\mu)\right),
\end{aligned}
$$


where we recall that $\mathfrak{p}=\mathfrak{g l}(m \mid \infty)_{\geq 0}$. Since $U\left(\mathfrak{g l}(m \mid \infty)_{-1}\right) \cong \Lambda\left(\mathbb{C}^{m *} \otimes \mathbb{C}^{\infty}\right)$ as a $\mathfrak{g l}(m) \oplus \mathfrak{g l}(\infty)$-module, $K(\lambda) \otimes K(\mu)$ has a Kac flag parameterized by the $\mathfrak{g l}(m) \oplus$ $\mathfrak{g l}(\infty)$-highest weights appearing in the decomposition of the module $\Lambda\left(\mathbb{C}^{m *} \otimes \mathbb{C}^{\infty}\right) \otimes$ $L^{0}(\lambda) \otimes L^{0}(\mu)$. By the skew-symmetric $(\mathfrak{g l}, \mathfrak{g l} \mathfrak{l})$-Howe duality [Ho] we have, as a $\mathfrak{g l}(m) \oplus \mathfrak{g l}(\infty)$-module,

$$
\Lambda\left(\mathbb{C}^{m *} \otimes \mathbb{C}^{\infty}\right) \cong \sum_{\gamma} L^{\mathfrak{g l}(m)}\left(\gamma^{*}\right) \otimes L^{\mathfrak{g l}(\infty)}\left(\gamma^{\prime}\right),
$$

where $\gamma$ is a partition with $\ell(\gamma) \leq m$, and $L^{\mathfrak{g l}(m)}\left(\gamma^{*}\right)$ denotes the $\mathfrak{g l}(m)$-module dual to $L^{\mathfrak{g l}(m)}(\gamma)$. Since the operator $-\sum_{i=-m}^{-1} e_{i i}$ provides an $\mathbb{N}$-gradation on $\Lambda\left(\mathbb{C}^{m *} \otimes \mathbb{C}^{\infty}\right)$ with each graded component consisting of only finitely many irreducible components, each irreducible $\mathfrak{g l}(m) \oplus \mathfrak{g l}(\infty)$-component in $\Lambda\left(\mathbb{C}^{m *} \otimes \mathbb{C}^{\infty}\right) \otimes$ $L^{0}(\lambda) \otimes L^{0}(\mu)$ appears with finite multiplicity. Thus $K(\lambda) \otimes K(\mu)$ admits an infinite filtration of Kac modules such that each Kac module appears with finite multiplicity. This implies that the product of $[K(\lambda) \otimes K(\mu)]$ in $\widehat{G}\left(\mathcal{O}_{m \mid \infty}^{++}\right)_{\mathbb{Q}}$ is well-defined.

Now consider the tensor product of two generalized Verma modules $\mathcal{K}\left(\lambda^{\natural}\right)$ and $\mathcal{K}\left(\mu^{\natural}\right)$. An analogous argument shows that $\mathcal{K}\left(\lambda^{\natural}\right) \otimes \mathcal{K}\left(\mu^{\natural}\right)$ has a filtration of generalized Verma modules parameterized by the irreducible $\mathfrak{g l}(m) \oplus \mathfrak{g l}(\infty)$-components appearing in the decomposition of $S\left(\mathbb{C}^{m *} \otimes \mathbb{C}^{\infty}\right) \otimes L^{0}\left(\lambda^{\natural}\right) \otimes L^{0}\left(\mu^{\natural}\right)$. By the symmetric $(\mathfrak{g l}, \mathfrak{g l} \mathfrak{l})$-Howe duality [으, as a $\mathfrak{g l}(m) \oplus \mathfrak{g l}(\infty)$-module,

$$
S\left(\mathbb{C}^{m *} \otimes \mathbb{C}^{\infty}\right) \cong \sum_{\gamma} L^{\mathfrak{g l}(m)}\left(\gamma^{*}\right) \otimes L^{\mathfrak{g l}(\infty)}(\gamma)
$$

where again the sum is over all partitions $\gamma$ with $\ell(\gamma) \leq m$. Thus the tensor product admits an infinite generalized Verma flag with each generalized Verma module appearing with finite multiplicity.

From the description of the $\mathfrak{g l}(m) \oplus \mathfrak{g l}(\infty)$-modules $\Lambda\left(\mathbb{C}^{m *} \otimes \mathbb{C}^{\infty}\right) \otimes L^{0}(\lambda) \otimes L^{0}(\mu)$ and $S\left(\mathbb{C}^{m *} \otimes \mathbb{C}^{\infty}\right) \otimes L^{0}\left(\lambda^{\natural}\right) \otimes L^{0}\left(\mu^{\natural}\right)$ above, it is clear that $[K(\lambda) \otimes K(\mu)]$ is mapped to $\left[\mathcal{K}\left(\lambda^{\natural}\right) \otimes \mathcal{K}\left(\mu^{\natural}\right)\right]$ under the map $\sharp$ given in Theorem 6.8. Thus, we have established the following.

Theorem 6.17. The natural isomorphism $\sharp: \widehat{G}\left(\mathcal{O}_{m+\infty}^{++}\right)_{\mathbb{Q}} \longrightarrow \widehat{G}\left(\mathcal{O}_{m \mid \infty}^{++}\right)_{\mathbb{Q}}$ is an isomorphism of Grothendieck rings. In particular, if $\lambda, \mu \in X_{m \mid \infty}^{+}$, then the composition factors of $L(\lambda) \otimes L(\mu)$ are in one-to-one correspondence with the composition factors of $\mathcal{L}\left(\lambda^{\natural}\right) \otimes \mathcal{L}\left(\mu^{\natural}\right)$ via $\sharp$.

\section{ACKNOWLEDGMENTS}

The first and third authors thank the University of Virginia for hospitality and support. The first author was supported by an NSC grant of the R.O.C. and is a member of the NCTS Taipei Office and the Tai-Da Institute for Mathematical Sciences, while the second author was supported by NSF. We thank Jon Brundan for helpful comments and references, and Bernard Leclerc for the very interesting and helpful reference LM. We thank Leonard Scott for helpful consultations on the KL theory. Finally we are greatly indebted to an anonymous expert for numerous thoughtful suggestions, criticism, and corrections that have resulted in this greatly improved version. 


\section{REFERENCES}

[BB] A. Beilinson and J. Bernstein, Localisation de g-modules, C. R. Acad. Sci. Paris Ser. I Math. 292 (1981), 15-18. MR610137 (82k:14015)

[BGS] A. Beilinson, V. Ginzburg and W. Soergel, Koszul duality patterns in representation theory, J. Amer. Math. Soc. 9 (1996), 473-527. MR1322847|(96k:17010)

[BK] J. L. Brylinski and M. Kashiwara, Kazhdan-Lusztig conjecture and holonomic systems, Invent. Math. 64 (1981), 387-410. MR632980 (83e:22020)

[BL] J. Bernstein and D. Leites, Character formulae for irreducible representations of Lie superalgebras of series $\mathfrak{g l}$ and $\mathfrak{s l}$, C.R. Acad. Bulg. Sci. 33 (1980), 1049-1051. MR620836 (82j:17020a)

[BR] A. Berele and A. Regev, Hook Young Diagrams with Applications to Combinatorics and to Representations of Lie Superalgebras, Adv. Math. 64 (1987), 118-175. MR884183 (88i:20006)

[Br1] J. Brundan, Kazhdan-Lusztig polynomials and character formulae for the Lie superalgebra $\mathfrak{g l}(m \mid n)$, J. Amer. Math. Soc. 16 (2003), 185-231. MR1937204 (2003k:17007)

[Br2] , Tilting modules for Lie superalgebras, Commun. Algebra 32 (2004), 2251-2268. MR2100468 (2005g:17014)

[CC] L. Casian and D. Collingwood, The Kazhdan-Lusztig conjecture for generalized Verma modules, Math. Z. 195 (1987), 581-600. MR900346 (88i:17008)

[CoI] D. Collingwood and R. Irving, A decomposition theorem for certain self-dual modules in the category $\mathcal{O}$, Duke Math. J. 58 (1989), 89-102. MR.1016415 (90k:17010)

[CPS] E. Cline, B. Parshall and L. Scott, Abstract Kazhdan-Lusztig theories, Tohoku Math. J. 45 (1993), 511-534. MR1245719 (94k:20079)

[CW] S.-J. Cheng and W. Wang, Howe Duality for Lie Superalgebras, Compositio Math. 128 (2001), 55-94. MR 1847665(2002h:17005)

[CZ] S.-J. Cheng and R. B. Zhang, Analogue of Kostant's u-cohomology formula for the general linear superalgebra, Internat. Math. Res. Not. 1 (2004), 31-53. MR2036954 (2005b:17042)

[Deo] V. Deodhar, On some geometric aspects of Bruhat orderings II: the parabolic analogue of Kazhdan-Lusztig polynomials, J. Algebra 111 (1987), 483-506. MR916182 (89a:20054)

[Don] S. Donkin, On tilting modules for algebraic groups, Math. Z. 212 (1993), 39-60. MR.1200163 (94b:20045)

[Dr] V. Drinfeld, Quantum groups, Proceedings of the ICM (Berkeley, 1986), 798-820, Amer. Math. Soc., Providence, RI, 1987. MR 934283 (89f:17017)

[Du] J. Du, IC bases and quantum linear groups, Proc. Symp. Pure Math. 56 (1994), 135-148. MR:1278732(95d:17010)

[ES] T. Enright and B. Shelton, Categories of highest weight modules: Applications to classical Hermitian symmetric pairs, Mem. Amer. Math. Soc. 67, no. 367 (1987). MR888703 (88f:22052)

[FKK] I. Frenkel, M. Khovanov and A. Kirillov, Jr., Kazhdan-Lusztig polynomials and canonical basis, Transform. Groups 3 (1998), 321-336. MR1657524 (2000f:20071)

[Ho] R. Howe, Perspectives on Invariant Theory: Schur Duality, Multiplicity-free Actions and Beyond, The Schur Lectures, Israel Math. Conf. Proc. 8, Tel Aviv (1992), 1-182. MR.1321638(96e:13006)

[Ir] R. S. Irving, Singular Blocks of the category O, Math. Z. 204 (1990), 209-224. MR1055986 (91i:17016)

[Ja1] J. Jantzen, Kontravariante Formen auf induzierten Darstellungen halbeinfacher LieAlgebren, Math. Ann. 226 (1977), 53-65. MR0439902 (55:12783)

[Ja2] _ Moduln mit einem höchsten Gewicht, Lect. Notes in Math. 750, Springer Verlag, 1983. MR552943(81m:17011)

[Ji] M. Jimbo, Quantum $R$ matrix for the generalized Toda system, Commun. Math. Phys. 102 (1986), 537-547. MR $824090(87 \mathrm{~h}: 58086)$

[JHKT] J. van der Jeugt, J. Hughes, R. King and J. Thierry-Mieg, A character formula for singly atypical modules of the Lie superalgebra $\mathfrak{s l}(m \mid n)$, Commun. Algebra 18 (1990), 3453-3480. MR 1063989 (91j:17046) 
[Jim] M. Jimbo, A q-analogue of $U(\mathfrak{g l}(N+1))$, Hecke algebra, and the Yang-Baxter equation, Lett. Math. Phys. 11 (1986), 247-252. MR841713(87k:17011)

[JZ] J. van der Jeugt and R. B. Zhang, Characters and composition factor multiplicities for the Lie superalgebra $\mathfrak{g l}(m \mid n)$, Lett. Math. Phys. 47 (1999), 49-61. MR1669394 (2000a:17008)

[K1] V. Kac, Lie Superalgebras, Adv. Math. 16 (1977), 8-96. MR0486011(58:5803)

[K2] - Representations of classical Lie Superalgebras, Lect. Notes in Math. 676, pp. 597-626, Springer Verlag, 1978. MR519631 (80f:17006)

[Kas] M. Kashiwara, On crystal bases of the Q-analogue of universal enveloping algebras, Duke Math. J. 63 (1991), 465-516. MR.1115118 (93b:17045)

[KL] D. Kazhdan and G. Lusztig, Representations of Coxeter groups and Hecke algebras, Invent. Math. 53 (1979), 165-184. MR.560412 (81j:20066)

[KMS] M. Kashiwara, T. Miwa, and E. Stern, Decomposition of q-deformed Fock spaces, Selecta Math. (N.S.) 1 (1995), 787-805. MR1383585 (97c:17021)

$[\mathrm{KT}] \quad$ S. Khoroshkin and V. Tolstoy, Universal R-matrix for quantized (super)algebras, Commun. Math. Phys. 141 (1991), 599-617. MR1134942 (93a:16031)

[KR] A. N. Kirillov and N. Reshetikhin, $q$-Weyl group and a multiplicative formula for universal R-matrices, Commun. Math. Phys. 134 (1990), 421-431. MR1081014|(92c:17023)

$[\mathrm{Ku}] \mathrm{J}$. Kujawa, Crystal structures arising from representations of $G L(m \mid n)$, preprint, math.RT/0311251. MR 2209849 (2006m:20018)

[LLT] A. Lascoux, B. Leclerc and J.-Y. Thibon, Hecke algebras at roots of unity and crystal bases of quantum affine algebras, Commun. Math. Phys. 181 (1996), 205-263. MR:1410572 (97k:17019)

[LS] A. Lascoux and M.-P. Schützenberger, Polynômes de Kazhdan et Lusztig pour les grassmanniennes, (French). Young tableaux and Schur functors in algebra and geometry (Toruń, 1980), Astérisque 87-88 (1981), 249-266. MR646823 (83i:14045)

[Lec] B. Leclerc, A Littlewood-Richardson rule for evaluation representations of $U_{q}\left(\widehat{\mathfrak{s l}}_{n}\right)$, Séminaire Lotharingien de Combinatoire, B50e, www.emis.ams.org/journals/SLC/.

[LM] B. Leclerc and H. Miyachi, Constructible characters and canonical bases, J. Algebra 77 (2004), 298-317. MR2059632 (2005g:17033)

[Lu1] G. Lusztig, Canonical bases arising from quantized enveloping algebras, J. Amer. Math. Soc. 3 (1990), 447-498. MR 1035415 (90m:17023)

[Lu2] Introduction to quantum groups, Progress in Math. 110, Birkhäuser, 1993. MR1227098 (94m:17016)

[MM] K. Misra and T. Miwa, Crystal base for the basic representation of $U_{q}(\widehat{\mathfrak{s l}}(n))$, Commun. Math. Phys. 134 (1990), 79-88. MR1079801 (91j:17021)

[PS] I. Penkov and V. Serganova, Representations of classical Lie superalgebras of type I, Indag. Math. (N.S.) 3 (1992), 419-466. MR1201236 (93k:17006)

[Se1] V. Serganova, Kazhdan-Lusztig polynomials for the Lie superalgebra $G L(m \mid n)$, Adv. Soviet Math. 16 (1993), 151-165. MR1237837(94k:17005)

[Se2] - Kazhdan-Lusztig polynomials and character formula for the Lie superalgebra $\mathfrak{g l}(m \mid n)$, Selecta Math. (N.S.) 2 (1996), 607-651. MR.1443186 (98f:17007)

[So1] W. Soergel, $\mathfrak{n}$-cohomology of simple highest weight modules on walls and purity, Invent. Math. 98 (1989), 565-580. MR1022307 (90m:22037)

[So2] - Kazhdan-Lusztig polynomials and a combinatoric for tilting modules, Represent. Theory 1 (1997), 83-114. MR1444322 (98d:17026)

[So3] - Character formulas for tilting modules over Kac-Moody algebras, Represent. Theory (electronic) 2 (1998), 432-448. MR1663141 (2000c:17048)

[So4] Character formulas for tilting modules over quantum groups at roots of one. Current developments in mathematics, 1997 (Cambridge, MA), 161-172, Int. Press, Boston, MA, 1999. MR1698856 (2000i:17040)

[St] E. Stern, Semi-infinite wedges and vertex operators, Internat. Math. Res. Notices, no. 4 (1995) 201-219. MR 1326065 (96b:17018)

[Sv] A. Sergeev, The tensor algebra of the identity representation as a module over the Lie superalgebras $\mathfrak{g l}(n \mid m)$ and $Q(n)$, Math. USSR Sbornik 51 (1985), 419-427. MR7735715 (85h:17010)

[Vo] D. Vogan, Irreducible representations of semsimple Lie groups II: The Kazhdan-Lusztig conjectures, Duke Math. J. 46 (1979), 805-859. MR.552528(81f:22024) 
[Wa] M. Wakimoto, Infinite-dimensional Lie algebras, Transl. Math. Monographs 195, Amer. Math. Soc., 2001. MR:1793723 (2001k:17038)

[Zou] Y.M. Zou, Categories of finite dimensional weight modules over type I classical Lie superalgebras, J. Algebra 180 (1996), 459-482. MR1378540(97e:17012)

Institute of Mathematics, Academia Sinica, Taipei, Taiwan 11529

E-mail address: chengsj@math.sinica.edu.tw

Department of Mathematics, University of Virginia, Charlottesville, Virginia 22904

E-mail address: ww9c@virginia.edu

School of Mathematics and Statistics, University of Sydney, New South Wales 2006, Australia

E-mail address: rzhang@maths.usyd.edu.au 